\title{
Roles for the adhesion molecule Contactin2 in the development and function of neural circuits in zebrafish
}

\author{
A Dissertation \\ presented to \\ the Faculty of the Graduate School \\ at the University of Missouri-Columbia \\ In Partial Fulfillment \\ of the Requirements for the Degree \\ Doctor of Philosophy
}

\begin{abstract}
by
Suman Gurung

Dr. Anand Chandrasekhar, Dissertation Supervisor
\end{abstract}

May 2018 
The undersigned, appointed by the dean of the Graduate School, have examined the dissertation entitled

\section{Roles for the adhesion molecule Contactin2 in the development and function of neural circuits in zebrafish}

presented by Suman Gurung,

a candidate for the degree of Doctor of Philosophy,

and hereby certify that, in their opinion, it is worthy of acceptance.

Dr. Anand Chandrasekhar

Dr. Andrew McClellan

Dr. Dawn Cornelison

Dr. Mark Hannink 


\section{ACKNOWLEDGEMENTS}

First, I would like to thank the two most important women in my life, my mother Goma Gurung and wife-to-be Priya Bariya. My mother was always there to hear my journey through graduate school, whether it be my failed experiments, my hectic academic schedule with teaching and research or eureka moments as I've worked on my thesis. I thank Priya, whose unconditional love is the source of my strength and happiness. I would also like to thank other members of my family for their love and support.

I cannot thank enough my advisor, Anand Chandrasekhar, for his guidance in my pursuit of this journey. By his example, he has shown me what a good scientist, teacher, and advisor should be. I would also like to thank my dissertation committee members, Dawn Cornelison, Andrew McClellan, and Mark Hannink who have provided me with excellent scientific suggestions as well as professional guidance.

My journey wouldn't be possible without the help and support of previous lab members: Vinoth Sittaramane, Xiufang Pan, Priyanka Singh, and Whitney Bryant. I owe much of my progress to them. I thank graduate students Emilia Asante and Devynn Hummel for making the lab a fun place to be even during the weekends. I appreciate all the hard work of Moe Baccam for keeping our zebrafish healthy and happy. I would like to thank all past and present undergraduates in the lab. Thanks to members from the Cornelison lab, 
especially Laura Arnold for her help with lab reagents and advice, in both academics and life.

Thank you to the zebrafish committee at Washington University at St. Louis for their suggestions and guidance in my project. Four hours of driving to and from Washington University was no match for the valuable suggestions provided by the committee.

I thank all my Nepalese friends here in Columbia for making this place home away from home and making me realize that there is life outside of lab and school. May the force be with them. 


\section{TABLE OF CONTENTS}

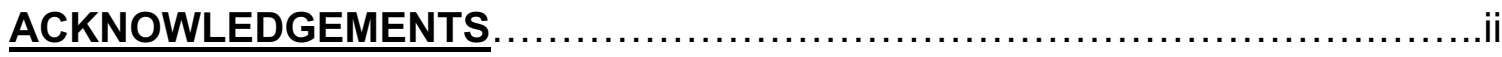

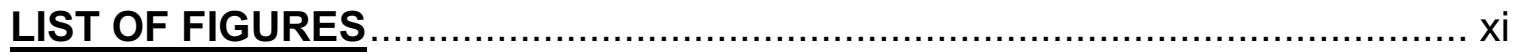

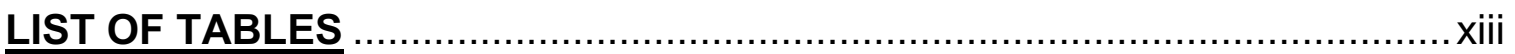

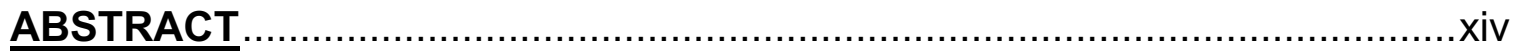

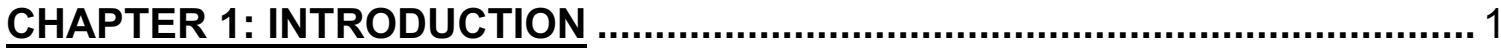

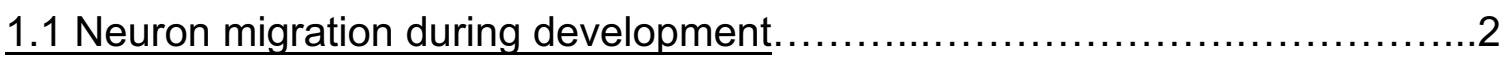

1.1.1 Radial Migration......................................................

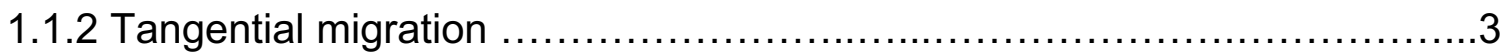

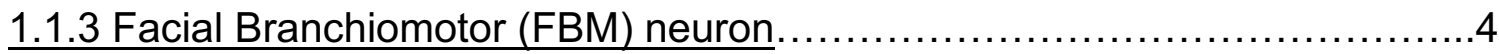

1.1.4 Mechanisms regulating tangential migration of facial branchiomotor

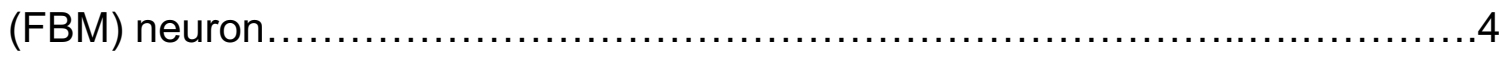

1.1.4.1 Cell adhesion molecules during FBM neuron migration..................5

1.1.4.2 The non-canonical Wnt/Planar cell polarity (PCP) pathway.............6

1.1.4.3 Role of vang/2 in tangential migration of FBM neurons..................

1.2 Genetic interaction between vangl2 and other PCP and non-PCP

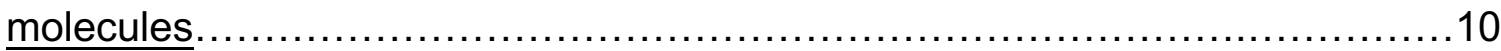

1.3 Axon guidance during development.........................................

1.3.1 Cellular and molecular mechanisms involved in axon guidance..........12

1.3.1a Guidance molecules............................................. 
1.3.2 Ig superfamily (IgSF) CAMs in axon guidance and fasciculation. 16

1.4 Cell types used to study axon guidance in zebrafish.

1.4.1 Interneurons of the nucleus of the medial longitudinal fascicle (nucMLF) 17

1.4.2 Rohon-Beard (RB) neurons ......................................... 19

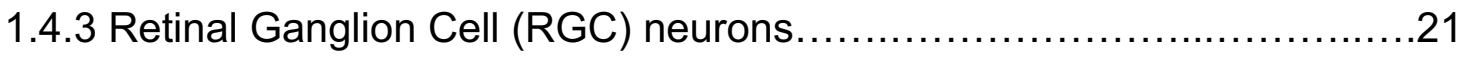

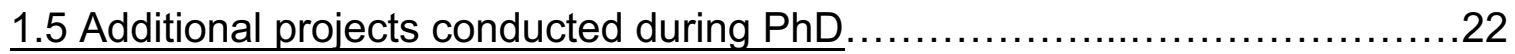

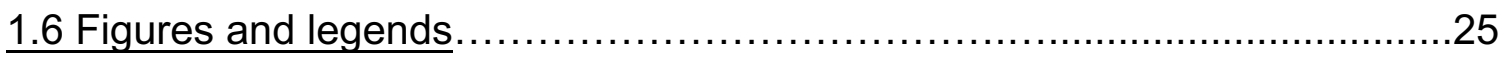

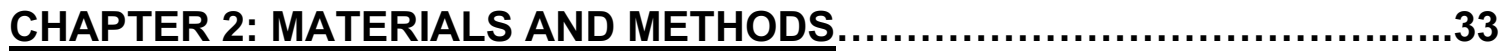

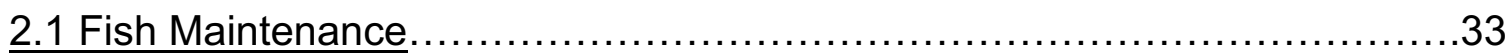

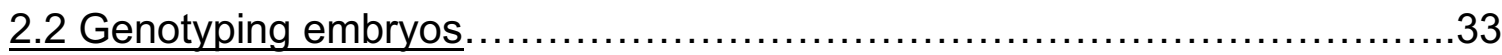

$\underline{2.3 \text { Genotyping Adults } \ldots \ldots \ldots \ldots \ldots \ldots \ldots \ldots \ldots \ldots \ldots \ldots \ldots \ldots \ldots \ldots \ldots \ldots \ldots \ldots \ldots \ldots \ldots \ldots \ldots \ldots \ldots \ldots \ldots}$

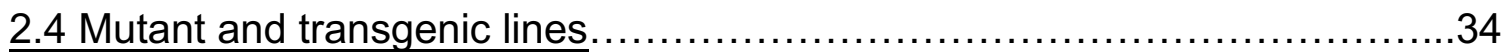

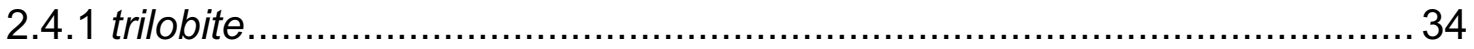

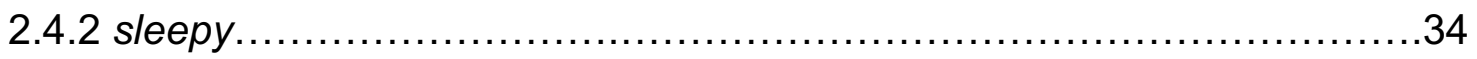

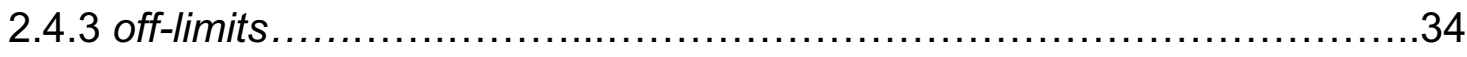

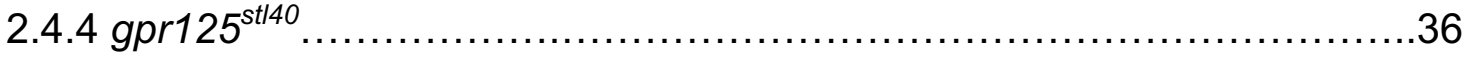

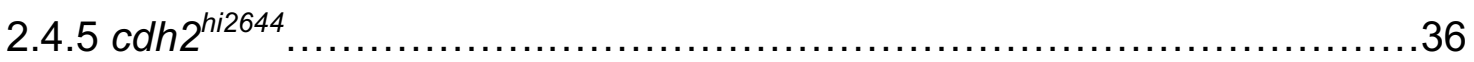

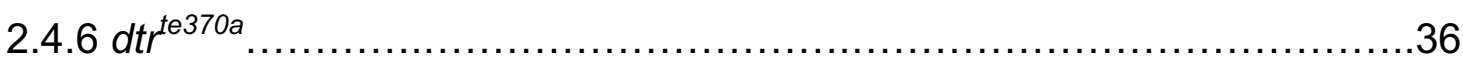

2.4.5 Islet1-GFP transgenic line $\operatorname{Tg}($ is/1:gfp) ................................

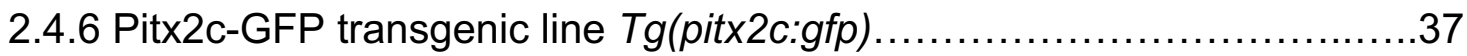


2.6.1 Design and synthesis of gRNA and Cas9 RNA.........................39

2.6.2 Test of sgRNA cleavage efficiency ...................................40

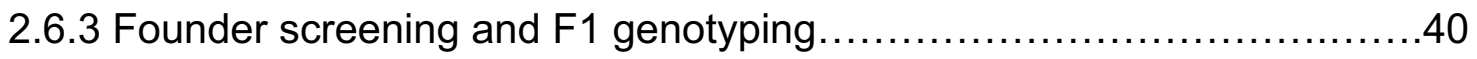

2.7 mRNA synthesis and RNA gel electrophoresis ....................................... 41

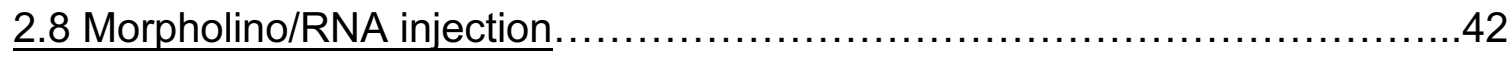

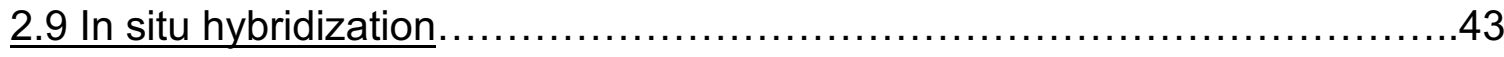

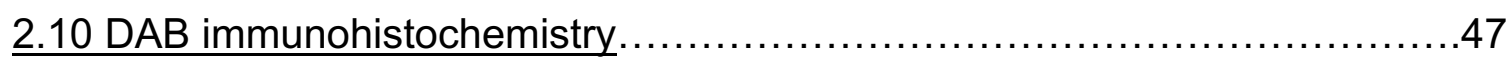

$\underline{2.11 \text { Fluorescent immunostaining } \ldots \ldots \ldots \ldots \ldots \ldots \ldots \ldots \ldots \ldots \ldots \ldots \ldots \ldots \ldots \ldots \ldots \ldots \ldots \ldots \ldots \ldots \ldots \ldots \ldots \ldots}$

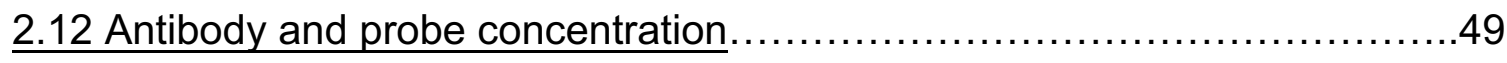

$\underline{2.13 \text { Quantification of FBM neuron } \ldots \ldots \ldots \ldots \ldots \ldots \ldots \ldots \ldots \ldots \ldots \ldots \ldots \ldots \ldots \ldots \ldots \ldots \ldots \ldots \ldots \ldots \ldots}$

2.14 Dil labeling of axons and photoconversion.................................49

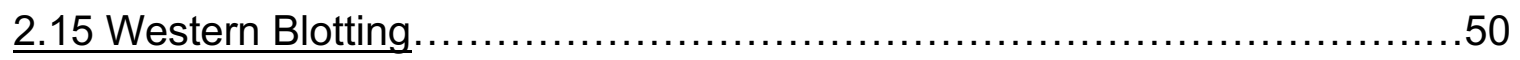

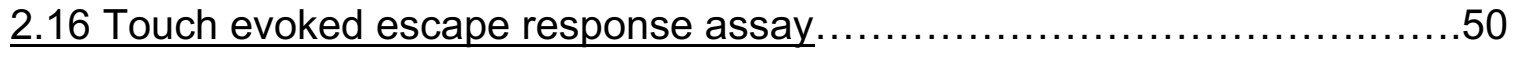

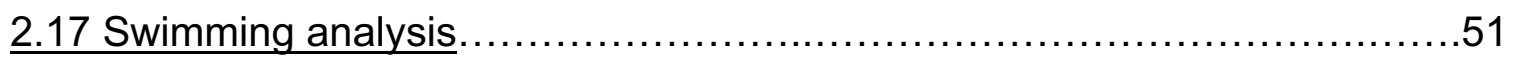




\section{CHAPTER 3: DISTINCT ROLES FOR THE CELL ADHESION MOLECULE CONTACTIN2 IN THE DEVELOPMENT AND FUNCTION OF NEURAL

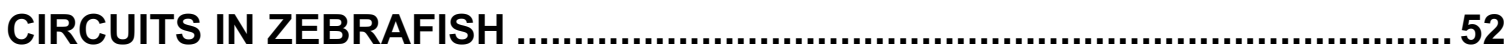

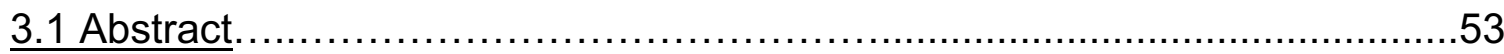

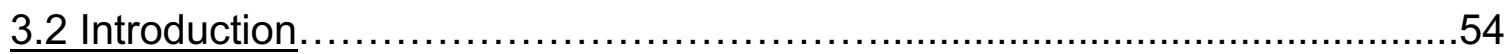

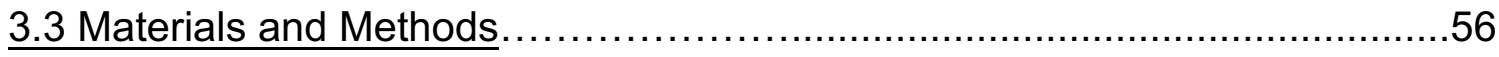

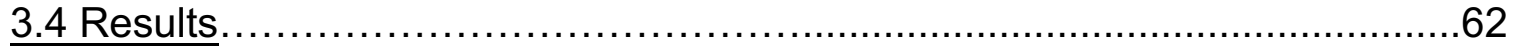

3.4.1 CRISPR-generated cntn2 alleles are null.............................62

3.4.2 FBM neurons migrate normally in cntn2 mutants.......................63

3.4.3 Genetic interaction between cntn2 and vang/2 for FBM neuron migration

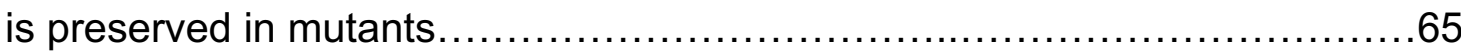

3.4.4 cntn2 mutants lack nucMLF axon convergence defects seen in morphants but exhibit MLF defasciculation................................67 3.4.5 FBM neuron and nucMLF defects in cntn2 morphants are likely off-target effects. 68

3.4.6 cntn2 mutants exhibit a defective escape response and have swimming deficits 69

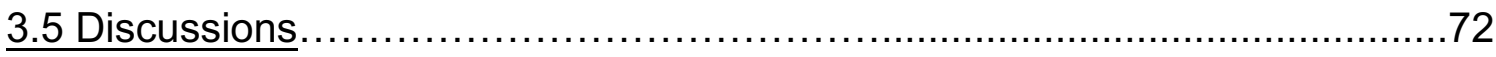

3.5.1 Comparison of cntn2 morphant and mutant phenotypes................72

3.5.2 Role for Cntn2 in FBM neuron migration..............................74

3.5.3 Role for Cntn2 in nucMLF development................................ 


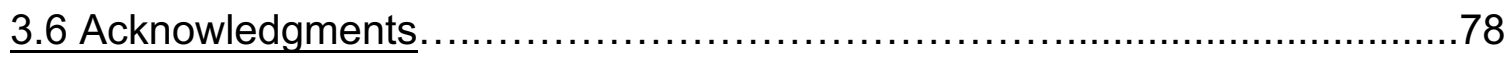

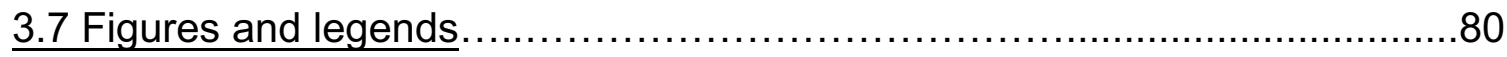

CHAPTER 4: DIFFERENTIAL ROLES FOR SPECIFIC PLANAR CELL POLARITY (PCP) AND NON-PCP GENES DURING FACIAL

BRANCHIOMOTOR NEURON MIGRATION IN ZEBRAFISH..................105

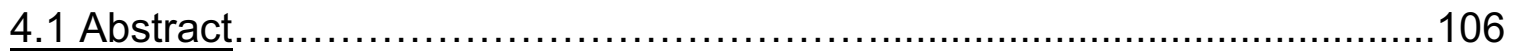

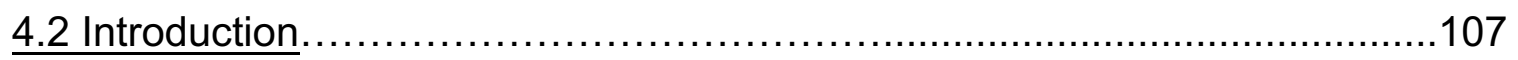

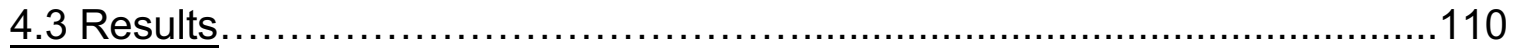

4.3.1 vangl2 genetically interacts with gpr125 for FBM neuron migration......110 4.3.2 vang/2 genetically interacts with cell adhesion gene cdh2 and PCP gene

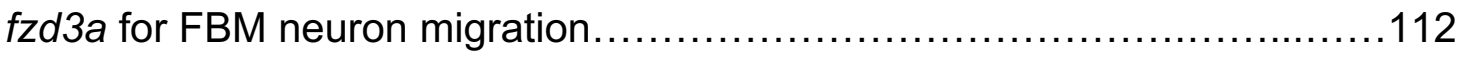

4.3.3 Other PCP and non-PCP genes do not exhibit genetic interactions for

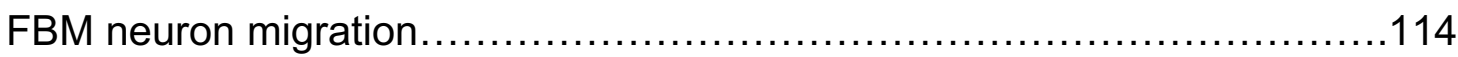

$\underline{4.4 \text { Discussion } \ldots \ldots \ldots \ldots \ldots \ldots \ldots \ldots \ldots \ldots \ldots \ldots \ldots \ldots \ldots \ldots \ldots \ldots \ldots \ldots \ldots \ldots \ldots \ldots \ldots \ldots \ldots \ldots \ldots \ldots \ldots \ldots \ldots \ldots \ldots \ldots \ldots}$

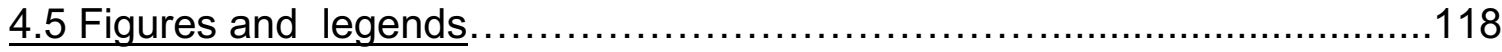

CHAPTER 5: CONCLUSIONS AND FUTURE DIRECTION $\ldots \ldots \ldots \ldots \ldots \ldots \ldots \ldots 126$

5.1 Do genetic interactions reflect physical interactions between membrane

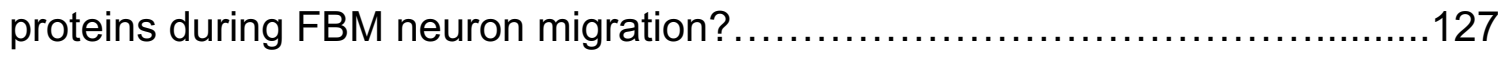

5.2 What structural motif in Cntn2 is important for MLF axon fasciculation?...128 5.3 Examine the function of genes misregulated in vang/2-deficient embryos.. 128

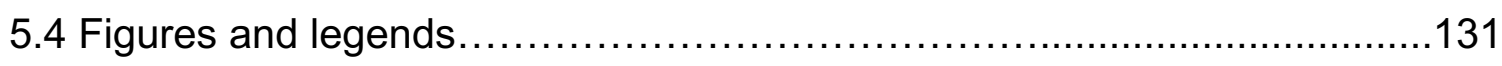


5.5 Tables.

\section{CHAPTER 6: A NOVEL GENETIC CELL ABLATION TECHNOLOGY IN}

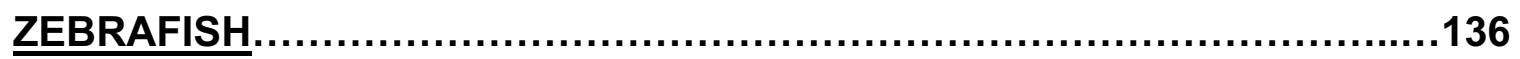

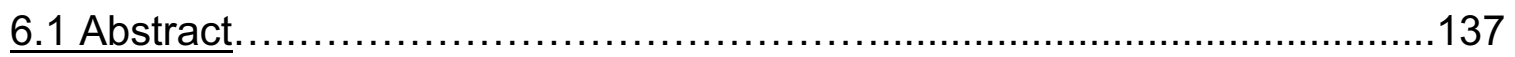

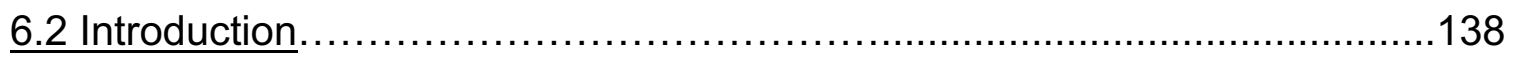

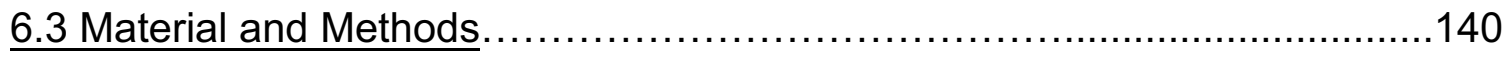

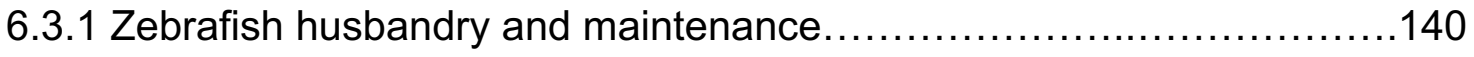

6.3.2 Testing for salt tolerance ........................................... 140

6.3.3 Assessing the effects of ILY treatment ................................141

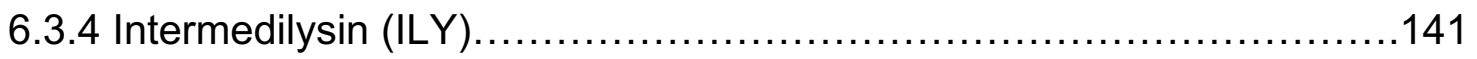

6.3.5 hCD59 constructs and RNA synthesis ..............................142

6.3.6 Validation of $h C D 59-m e d i a t e d$ cell ablation in transient expression assay

6.3.7 Generation of zCREST:hCD59 transgenic zebrafish ..................143

6.3.8 Primary culture of zebrafish embryonic neurons ......................144

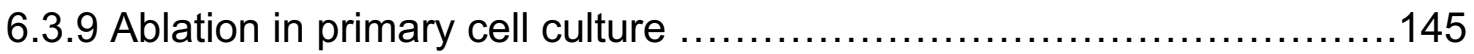

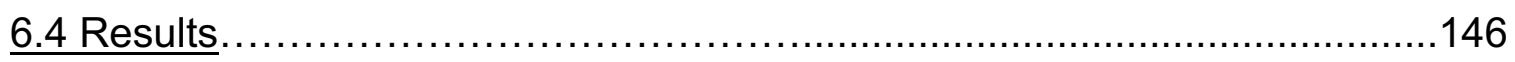

6.4.1 Establishing optimal conditions for testing the efficacy of ILY treatment in

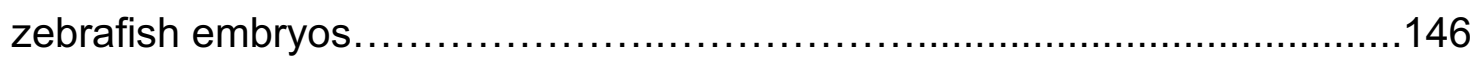

6.4.2 ILY induces mortality in hCD59-expressing embryos, and requires the

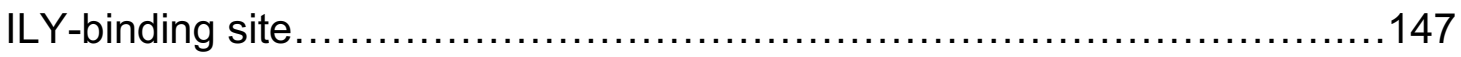

6.4.3 ILY-induced ablation of a specific cell type in transgenic embryos.......148 


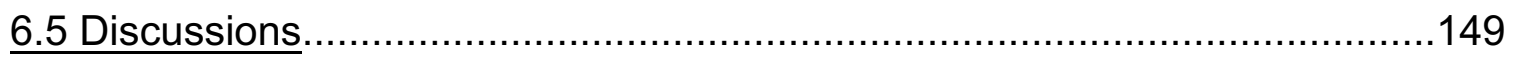

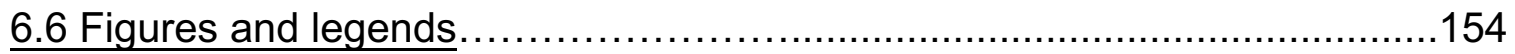

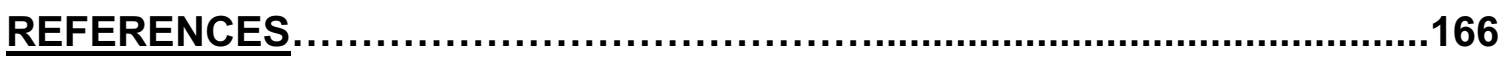

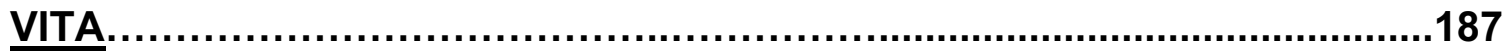




\section{LIST OF FIGURES}

Figure 1.1 Radially migrating neurons moving along radial glia processes in three

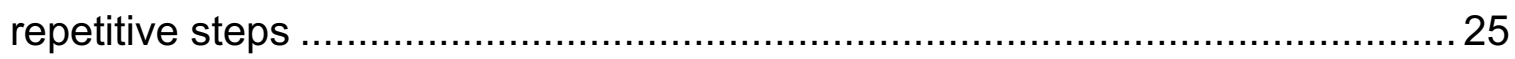

Figure 1.2 Facial branchiomotor (FBM) neuron migration in zebrafish ..............27

Figure 1.3 MLF axons extension and convergence zebrafish .........................29

Figure 1.4 Rohon-Beard neurons in zebrafish trunk ..................................... 31

Figure 3.1 Cntn2 protein expression during embryonic developmetnt ............... 80

Figure 3.2 Generation and validation of CRISPR-generated cntn2 mutant........82

Figure 3.3 Knock down of Cntn2 expression with antisense MO.................84

Figure 3.4 FBM neuron migration is affected in cntn2 morphants but not in cntn2

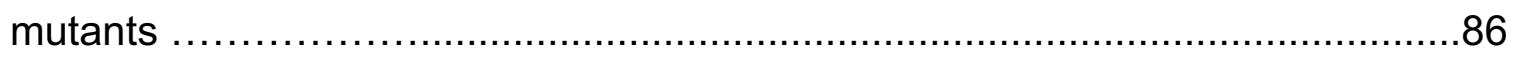

Figure 3.5 cntn2 interacts genetically with vangl2 but not with lamc1 $\ldots \ldots \ldots \ldots \ldots . .88$

Figure 3.6 cntn2 mutants show MLF defasciculation but lacks nucMLF defects

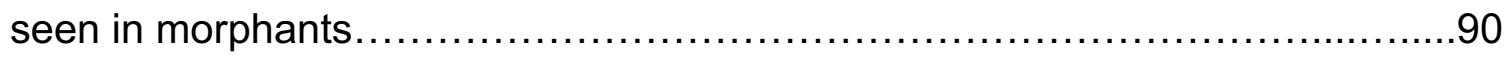

Figure 3.7 Some neuronal defects in cntn2 morphants are likely to be off-target

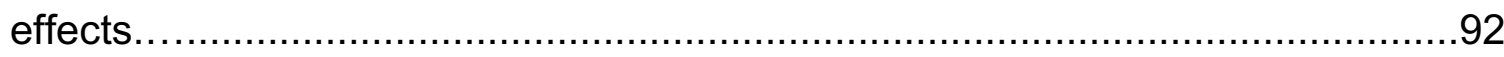

Figure 3.8 Outgrowth of Rohon-Beard central axons is not affected in MZcntn2

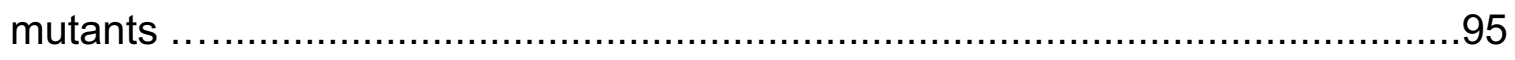

Figure 3.9 cntn2 mutants exhibit defective touch responses ......................... 97

Figure 3.10 Spinal motor axons develop normally in cntn2 mutants .................. 99 
Figure 3.11 cntn2 mutants exhibit swimming deficits.

Figure 3.12 Retinal ganglion cell axon fascicles are variably affected in cntn2

mutants

Figure 4.1 vangl2 genetically interacts with gpr125 for FBM neuron

migration

Figure 4.2 vangl2 genetically interacts with cell adhesion genes cdh2 and PCP

gene $f z d 3 a$ for FBM neuron migration 120

Figure 4.3 Other PCP and non-PCP genes do not exhibit genetic interactions for FBM neuron migration. 122

Figure 4.3 Summary of pairwise genetic interaction analysis 124

Figure 5.1 Tagged constructs and their detection by western blotting.... .131

Figure 5.2 Expression pattern of candidate genes identified from transcriptomic

analysis 133

Figure 6.1 Assessment of high salt and ILY-induced mortality 154

Figure 6.2 Mortality upon exposure of mRNA injected embryos to ILY 156

Figure 6.3 Cell lysis upon treatment of WT hCD59-expressing embryos with ILY 158

Figure 6.4 Generation of transgenic line employed in this study.... 160

Figure 6.5 ILY treatment in transgenic embryos 162

Figure 6.6 ILY induces rapid ablation of hCD59-expressing cultured neurons 164 


\section{LIST OF TABLES}

Table 5.1 CRISPR/Cas9 target site and oligonucleotides used to make gRNA for

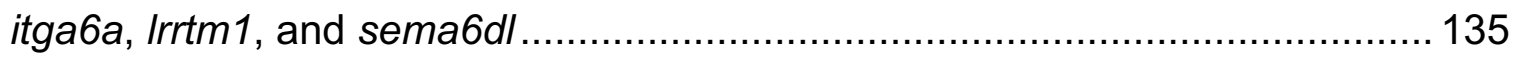

Table 5.1 Summary of CRISPR/Cas9 generated alleles of ita6a, Irrtm1, and

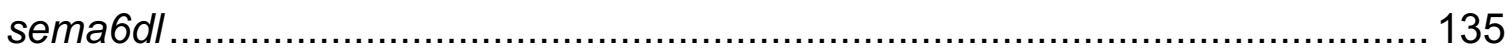




\section{ABSTRACT}

Neuronal migration and axon guidance are critical developmental processes that are essential for establishing functional neural circuits underlying complex cognitive and motor functions. Precise neuronal migration and axon guidance are dependent upon cell-cell and cell-substrate interactions, which are mediated by several membrane-associated molecules. The relatively concise segmental organization of the hindbrain and the simple scaffold of axon tracts in the zebrafish brain provides an ideal model to study how different molecules collaborate to guide migrating neurons and growing axons to their final location. In this thesis, I examine the roles of membrane molecules during neuron migration and axon guidance in zebrafish. In Chapter 3, I show the generation of Contactin2 (Cntn2) null mutant using CRISPR/Cas9 and characterize cntn2 mutant. I demonstrate a role for cntn2 in facial branchiomotor (FBM) neuron migration and fasciculation of medial longitudinal fascicle (MLF) axons. In addition, using touch-evoked escape response and swimming assays, I show sensorimotor deficits in cntn2 mutants. Collectively, these data demonstrate distinct developmental roles for zebrafish cntn2 in neuronal migration and axon fasciculation, and in the function of sensorimotor circuits. In Chapter 4, I examine pairwise genetic interactions between several PCP and non-PCP genes for FBM neuron migration. I show that vang/2 is rather unique in exhibiting genetic interactions with several PCP and non-PCP genes. These data suggest that 
vang/2 might be playing a central role in regulating the function of many PCP and non-PCP genes for FBM neuron migration. In Chapter 6, I describe a novel genetic approach which utilizes the human CD59 receptor (hCD59) and the bacterial toxin intermedilysin (ILY) for rapid cell ablation in zebrafish. 


\section{CHAPTER 1: INTRODUCTION}

A major challenge in neuroscience is to generate a full understanding of the cellular and molecular mechanisms underlying the formation of functional networks in the central nervous system. Establishment of functional neuronal circuits depends on the ability of specific populations of neurons to form appropriate synaptic connections with target neurons or non-neuronal cells. During the development of the nervous system these connections are formed through a series of consecutive events. First, the process of neural induction and differentiation give rise to different classes of neurons from progenitor cells (Jessell, 2000). Following neuronal differentiation, immature neurons migrate from their birthplace to their final positions (Hatten, 2002). Upon reaching to their final location, neurons extend axons, which navigate through the developing brain by responding to various cellular and extracellular (diffusible) cues to reach to their specific targets (Tessier-Lavigne and Goodman, 1996), and establish synapses. Finally, synaptic refinement takes place which involves the reorganization of synapses and connections. These events are important for the establishment of functional neural circuits that govern complex motor and cognitive functions.

In this chapter, I will briefly review molecular mechanisms regulating neuronal migration and axon guidance. Next, I will review three topics that figure prominently in my studies: 1) the migration of facial branchiomotor (FBM) neurons in the hindbrain, 2) the outgrowth and organization of axons of the nucleus of the medial longitudinal fasciculus (nucMLF) and Rohon-Beard (RB) neurons in the midbrain and spinal cord, respectively, and 3) the fasciculation of retinal ganglion cell (RGC) axons. 


\subsection{NEURONAL MIGRATION DURING DEVELOPMENT}

The function of a neural circuit depends on the precise connections made by neurons with their targets. Neuronal migration is a ubiquitous feature of nervous system development that brings neurons into appropriate spatial relationships with each other and non-neuronal tissue. During development of the nervous system, newborn neurons migrate away from the germinal layer to locations where they form functional neural circuits. A migratory neuron must make several decisions: whether to migrate, what direction to migrate in, and when to terminate migration. Neuronal migration is largely dependent on the ability of the migrating neurons to interact with neighboring cells and with their extracellular environment to sense and respond to different migration signals.

In the developing neural tube, neuronal precursors move in the plane of the neuroepithelium called the ventricular zone (VZ). Neuroepithelial cells after being specified as neurons, begin to differentiate and migrate away from the $V Z$ along distinct radial and tangential pathways to their final destinations (Hatten, 1999; Parnavelas et al., 2002). Neuronal migration can be classified into radial and tangential modes based on the direction in which the neurons migrate relative to the surface of the CNS.

\subsubsection{Radial Migration}

During radial migration, neurons translocate from the $V Z$ to the more superficial layers in the brain through the radial glial fibers (Hatten, 1999). Radial migration is the most extensively studied form of neuronal migration in the CNS. Radially migrating 
neurons are closely associated with the radial glial processes, which are the substrate upon which these neurons migrate (Rakic, 1974). Radially migrating neurons exhibit a bipolar morphology and move along the processes of glial cells in three repetitive steps. They first extend a leading process in the direction of migration (Rivas and Hatten, 1995) which is followed by nucleokinesis, or nuclear translocation. Nucleokinesis begins with the forward movement of the centrosome into the leading process. The final step involves retraction of the trailing process at the rear of the cell (Horwitz and Parsons, 1999) (Fig. 1.1).

\subsubsection{Tangential migration}

While most neurons migrate radially to reach to their final destinations, a significant number of cortical and other neurons also migrate, but independently of radial glia (Chandrasekhar, 2004; Hatten, 1999; Lois et al., 1996; Marin and Rubenstein, 2003; Pearlman et al., 1998). This glia-independent migration, which occurs along axes orthogonal to the direction of radial migration, is called tangential migration. In contrast to radial migration, which generates distinct neuronal layers or clusters, tangential migration generates neuronal diversity within a given brain region (Marin and Rubenstein, 2003). The molecular and cellular mechanisms governing the tangential migration are less well understood when compared to radial migration.

Due to their conserved organization as well as the stereotypic migration, the facial branchiomotor (FBM) neurons have become an excellent model to study neuronal 
migration in the vertebrate embryo (Chandrasekhar, 2004; Song, 2007). Branchiomotor neurons are generated in specific rhombomeres in the hindbrain and innervate muscles that arise in the pharyngeal arches (Chandrasekhar et al., 1997).

\subsubsection{FACIAL BRANCHIOMOTOR (FBM) NEURONS}

The FBM neurons are a subset of cranial motor neurons found in the vertebrate brainstem (Chandrasekhar, 2004; Gilland and Baker, 2005). In mammals, the facial motor neurons innervate muscles of facial expression, and of the middle ear and upper neck. In all vertebrates investigated, except chicken, FBM neurons undergo a complex and stereotyped tangential migration in the hindbrain along the rostral-caudal axis from rhombomere 4 (r4) to r6 and r7 (Chandrasekhar, 2004) (Fig. 1.2A). Stable transgenic lines, such as the $T g(i s / 1: G F P)$ strain that labels all branchiomotor neurons (Higashijima et al., 2000), allow for the visualization of distinct cell populations in live embryos (Fig. $1.2 \mathrm{~B})$.

\subsubsection{Mechanisms regulating tangential migration of facial branchiomotor (FBM) neurons}

Tangential migration of FBM neurons is highly dependent on environmental cues as well as cell-intrinsic mechanisms. Several factors have been shown to cellautonomously regulate the ability of the motor neurons to respond to environmental cues, including transcription factors like r4-expressed Hoxb1 and FBM neuron- 
expressed Ebf, Nkx6.1, Hdac1 and Tbx20 (Garel et al., 2000; Muller et al., 2003;

Nambiar et al., 2007; Pocock et al., 2008; Song, 2007). Conversely, loss of

rhombomere identity in Krox20 (expressed in r3 and r5) and Kreis/er/valentino/MafB (expressed in r5 and r6) mutants leads to defective FBM neuron migration, likely a noncell autonomous effect due to the loss of environmental cues (Chandrasekhar et al., 1997; Garel et al., 2000). Studies in zebrafish have identified several genes necessary for FBM neuron migration from $\mathrm{r} 4$ to $\mathrm{r} 6$ and $\mathrm{r} 7$. Chromatin regulatory proteins Hdac1 (Nambiar et al., 2007) and REST (Mapp et al., 2011), elongation factor Foggy/Spt5 (Cooper et al., 2005), stromal cell-derived factor Sdf1a/Cxcl1a its receptor CxcR4 and CxcR7 (Cubedo et al., 2009), the autism susceptibility protein Met and its Hgf ligand (Elsen et al., 2009), adhesion GPCR gpr125 (Li et al., 2013), the cell adhesion molecule Cntn2 (Sittaramane et al., 2009) and ECM molecules, Laminina1 and Y1 (Grant and Moens, 2010; Sittaramane et al., 2009), are necessary for FBM neuron migration.

\subsubsection{Cell adhesion molecules with potential roles in FBM}

\section{neuron migration}

During migration, neurons interact with signals found on neighboring cells and the extracellular matrix (ECM). Laminins are heterotrimeric protein complexes and can modulate a neuron's response to extracellular guidance molecules. In zebrafish, Laminina1 (lama1), a broadly expressed ECM molecule, plays a role in FBM neuron migration (Paulus and Halloran, 2006; Sittaramane et al., 2009). Consistent with this, 
morpholino-based analyses have identified itga6a and itgb1, which respectively encode IntegrinA6 and IntegrinB1 subunits of a Laminin-binding receptor, as potential regulators of FBM neuron migration (V. Sittaramane, S. Gurung, and A. Chandrasekhar, unpublished data). Mutations in celsr2, which encodes an atypical Cadherin, cause severe defects in FBM neuron migration (Wada et al., 2006). Similarly, disruption of neural cell adhesion molecule Cdh2 expression or function generated migration defects where most of the FBM neurons failed to migrate out of r4 (Rebman et al., 2016; Stockinger et al., 2011). We previously showed that the cell adhesion molecule Contactin-2 (Cntn2)/ Transient Axonal Glycoprotein (Tag1) is necessary for the migration of the FBM neurons in the zebrafish (Sittaramane et al., 2009). Together, these studies highlight the importance of cell-cell and cell-substrate interactions during FBM neuron migration. However, some of these data, including the cntn2 results, were obtained using antisense morpholinos, with possibilities of off-target effects and toxicity (Eisen and Smith, 2008). Therefore, we generated loss-of-function mutations in cntn2 using CRISPR/Cas9 to address potential concerns with the MO knockdown studies and to investigate roles for zebrafish Cntn2 at later developmental stages in Chapter 3.

\subsubsection{The non-canonical Wnt/Planar cell polarity (PCP) pathway and FBM neuron migration}

Wnts are secreted glycoproteins that bind to Frizzled (Fz), a seven-pass transmembrane receptor. Binding of Wnts to Fz activates one of two signaling 
pathways, a canonical pathway that allows $\beta$-catenin to mediate transcriptional changes (Logan and Nusse, 2004), and a non-canonical pathway that activates downstream ROCK and Jun kinases altering cytoskeleton arrangements or cell polarity. Planar Cell Polarity (PCP) signaling has been primarily studied in the context of the polarization of epithelial tissues. The non-canonical Wnt pathway has been shown to regulate both cell polarity and movements of dorsal mesodermal cells during convergent extension and also later during neural tube closure (Veeman et al., 2003). In zebrafish, Wnt/PCP signaling regulates convergence and extension during movements during vertebrate gastrulation (Roszko et al., 2009) and aids in establishing the polarity of neural progenitors (Ciruna et al., 2006). The importance of PCP signaling in regulating FBM neuron migration has been well demonstrated. Mutation in Wnt/PCP components like Van gogh-like 2 (Vangl2) (Bingham et al., 2002; Glasco et al., 2012; Jessen et al., 2002;

Vivancos et al., 2009), Prickle1a (Pk1a) (Carreira-Barbosa et al., 2003), Prickle1b (Pk1b) (Rohrschneider et al., 2007), Scribble1 (Scrib1) (Wada et al., 2005), Frizzled3a and Celsrs 1-3 (Qu et al., 2010; Wada et al., 2006) leads to FBM neuron migration defects in zebrafish and mouse. However, other components of PCP pathway do not appear to play roles in FBM neuron migration. (Disheveled, Wnts, Rho Kinase)

\subsubsection{Role of vang/2 in tangential migration of FBM neurons}

The trilobite locus encodes Van gogh-like 2 (Vangl2) (Jessen et al., 2002), a core component of Wnt/PCP pathway (Veeman et al., 2003). Vangl2 is a four-pass membrane-spanning protein with the $\mathrm{N}$-terminal and $\mathrm{C}$-terminal domains located in the 
cytosol, with a PDZ domain-binding motif (PBM) at the C-terminus (Darken et al., 2002;

Katoh, 2005; Park and Moon, 2002). The tangential migration of FBM neurons from r4 to r6 and r7 is eliminated in vang/2-/- mutants (Bingham et al., 2002; Jessen et al., 2002). vang/2 is expressed broadly in the hindbrain, and largely functions nonautonomously for FBM neuron migration (Jessen et al., 2002). During my graduate work, I contributed to two projects involving the 1) identification of cell-type where Vangl2 functions and 2) examination of the roles of Vangl2 domains in FBM neuron migration. I have briefly summarized the papers below.

\subsection{Identification of cell-type where Vangl2 functions}

\section{(Sittaramane et al., 2013)}

Vangl2 is broadly expressed in zebrafish hindbrain, and it functions largely noncell autonomously to regulate FBM neuron migration (Jessen et al., 2002). However, the cell-type within which Vangl2 acts is unknown. Using targeted transplantations and cell type-specific transgene expression, we demonstrated that vangl2 functions primarily in floor plate cells in $\mathrm{r} 4$ to regulate migration. Importantly, we ruled out a role for vangl2 in FBM neurons for mediating motor neuron-motor neuron interactions (collective migration). FBM neurons frequently contact floor plate cells in $\mathrm{r} 4$, and a biologically active GFP-Vangl2 fusion protein is enriched on floor plate cell membranes. These observations suggest that Vangl2 present on floor plate membranes facilitates signaling necessary for initiating FBM neuron migration 


\subsection{Roles of Vangl2 domains in FBM neuron migration (Pan et al., 2014)}

In vertebrates, Vangl2 is necessary for developmental and physiological processes including convergence and extension (CE) movements during gastrulation (Marlow et al., 1998), wound repair (Caddy et al., 2010), tumor cell migration (Cantrell and Jessen, 2010), and orientation of cilia in many tissues and organs (Borovina et al., 2010; May-Simera et al., 2010; Song et al., 2010). However, despite its broad roles in development and disease, the roles of various domains of Vangl2 in specific cellular behaviors such as FBM neuron migration have not been studied. We, therefore, wanted to examine the roles of specific Vangl2 domains in the context of FBM neuron migration. To do this, we generated various myc-tagged Vangl2 variants lacking specific domains and asked whether these Vangl2 variants could rescue FBM neuron migration defects of vang/2-/- mutants by RNA injection. However, this strategy was not feasible because RNA injection of several constructs generated CE defects. To bypass CE defects induced by RNA injection, we generated transgenic lines for heat shock-inducible expression of the Vangl2 variants. Surprisingly, migration of FBM neurons was strongly inhibited in heat-shocked transgenic embryos, a dominant-negative phenotype not observed following RNA injection. This unexpected dominant negative effect of Vangl2 overexpression precluded an analysis of the various Vangl2 domains to rescue FBM neuron migration in vang/2-/- mutants. Therefore, instead of testing whether the Vangl2 
variants could rescue FBM neuron migration in vang/2-/- mutants, we tested their abilities to generate the dominant-negative phenotype. We found that membrane associated functions of the $\mathrm{N}$-terminal and C-terminal domains of Vangl2 are involved in regulating FBM neuron migration. Importantly, using temperature shifts, we determined that Vangl2 function is required between $15-20 \mathrm{hpf}$, coinciding with the onset of migration, and suggesting that Vangl2 is required for the initiation of FBM neuron migration in $\mathrm{r} 4$.

\subsection{Genetic interaction between vang/2 and other PCP and}

\section{non-PCP molecules for FBM neuron migration}

Previous studies have shown that vangl2 functions with knypek (glypican4/6), pipetail (wnt5a), silberblick (wnt11) and disheveled (Dsh) to regulate convergence and extension movements in Wnt/PCP pathway (Jessen et al., 2002; Park and Moon, 2002). However, there are multiple lines of evidence suggesting that the role of vang/2 in FBM neuron migration is independent of its function in the Wnt/PCP pathway. 1) Several trilobite alleles only generate mild CE defects but exhibit severe FBM neuron migration defects. 2) vangl2 functions cell autonomously for CE movements and non-cell autonomously for FBM neuron migration (Jessen et al., 2002). (3) Mutations in other PCP molecules such as knypek (glypican4/6), pipetail (wnt5a) and silberblick (wnt11) produce CE defects, but do not affect FBMN migration (Jessen et al., 2002; Park and Moon, 2002). These observations suggest strongly that vang/2 may function through the 
novel, Wnt/PCP-independent cellular and molecular mechanisms to mediate FBM neuron migration. Interestingly, we showed previously that cntn2 (a non-PCP gene) and vang/2 genetically interact during FBM neuron migration since injection of a suboptimal dose of cntn2 $\mathrm{MO}$ enhances the weak FBM neuron migration phenotype of vang/2 heterozygotes (Sittaramane et al., 2009). This result suggests that Cntn2 and Vangl2 participate in a common mechanism to regulate FBM neuron migration. Similarly, vangl2 has also been shown to genetically interact with gpr125 (a non-PCP gene) for FBM neuron migration ( $\mathrm{Li}$ et al., 2013). Together, these data suggest that vang/2 may interact with other non-PCP molecules expressed in the FBM neurons or in the environment to regulate FBM neuron migration. However, these genetic interaction data were obtained from morpholino studies with possibilities of off-target effects and toxicity (Eisen and Smith, 2008). We, therefore, aimed to validate the genetic interaction between vang/2 and cntn2, and vang/2 and gpr125 using genetic mutants in chapter 3 and 4. We also tested for genetic interaction between vang/2 and other PCP and nonPCP genes for FBM neuron migration.

\subsection{AXON GUIDANCE DURING DEVELOPMENT}

During development of the nervous system, axons follow very precise pathways to reach their destination. Axons reach their target by integrating many external signals. The correct wiring of the nervous system depends on the ability of axons to locate and recognize their appropriate synaptic partners. Axons are equipped with motile and 
highly sensitive structures called growth cones to help navigate their way. Growth cones sense guidance cues, both attractive and repulsive, in the environment by extending fingerlike projections called filopodia. These structures use guidance cues as their "road signs" to reach their appropriate targets. Growth cone extension, retraction, or turning occurs from the rearrangement of the growth cone's cytoskeleton and the modulation of growth cone-substrate adhesivity. Neighboring cells, as well as axons, provide guidance cues to growing axons. Together, two intimately associated processes: 1) axon guidance and 2) axon growth help to establish appropriate synaptic connections, generate topographic maps and define receptive fields during the critical periods. Misregulation in this process could cause synaptic dysfunctions and lead to impaired cognitive and mental abilities seen in many developmental as well as psychiatric disorders (Engle, 2010).

\subsubsection{Cellular and molecular mechanisms involved in axon}

\section{guidance}

Proper assembly of functional neural circuits requires tight regulation of axon growth and guidance. With the technological advances in molecular genetics, highresolution imaging, and neuronal culture, tremendous progress has been made in elucidating the molecular and cellular mechanisms underlying pathway selection and target recognition. Over the past years, a plethora of molecules has been identified that regulate axon growth and guidance during the development of the nervous system. 


\subsection{1a Guidance molecules}

Guidance cues include molecules from several gene families that are conserved across many species. These molecules are either expressed in the environment for long-range communication or on the cell surface for short-range communication between surrounding cells and growth cones. Four families of guidance cues were originally identified that mediate axons guidance.

\subsection{1a1 Netrins}

Netrin (Net) proteins are a family of evolutionarily conserved laminin-related diffusible proteins that are secreted by the floor plate to attract commissural axons to cross the midline (Kaprielian et al., 2001; Serafini et al., 1994). Vertebrate Netrins were first identified in the Chick, where they function to promote commissural axons outgrowth and guidance toward the floor plate (Serafini et al., 1996). Netrins have been shown to regulate axon guidance in many organisms, from nematodes and insects to higher mammals (Kaprielian et al., 2001). Commissural axons exhibit pathfinding defects and fail to reach the floor plate in mice lacking functional netrin (Serafini et al., 1996). UNC-5 and DCC (Deleted in Colorectal Carcinoma) have been identified as receptors for netrins through biochemical and genetic studies in several different species (Culotti and Merz, 1998; Hong et al., 1999). DCC receptors largely mediate attraction to netrins but can also participate in repulsion. UNC-5 receptors exclusively 
function in repulsion which is mediated by UNC-5 alone or in combination with DCC receptors (Hedgecock et al., 1990; Hong et al., 1999).

\subsection{1a2 Slits}

Slits are large secreted glycoproteins first discovered in a genetic screen of embryonic patterning defects in Drosophila (Nusslein-Volhard et al., 1984). Their role in axon guidance was later observed in genetic screens for commissural axon guidance pathfinding defects in Drosophila (Hummel et al., 1999). Slits homologs have been characterized in C. elegans (Hao et al., 2001), Xenopus (Chen et al., 2000), zebrafish (Hutson and Chien, 2002), chick (Holmes and Niswander, 2001), as well as in mammals (Brose et al., 1999). Slits signal through Roundabout (Robo) family receptors. Gene encoding these receptors were first identified in Drosophila in a genetic screen for genes regulating midline guidance defects in the central nervous system (CNS).

Genetic and biochemical studies showed that Slit can bind to Robo, which functions as midline repellent (Brose et al., 1999; Kidd et al., 1998). Slit mutants exhibit collapse of axon tracts onto the midline in Drosophila (Kidd et al., 1998). Similarly, in vertebrate slit mutant, commissural axons show defects in midline crossing (Long et al., 2004). Interestingly, Robo mutants also show defects in commissural axon pathfinding (Kidd et al., 1998).

\subsection{1a3 Semaphorins}


Semaphorins are a large family of cell surface and secreted guidance molecules. Semaphorins were first identified by searching for molecules expressed on specific axon fascicles. Molecules of this family share a conserved "sema" domain and are divided into eight classes based on their origin and structural elements (Raper, 2000). Semaphorins signal through multimeric receptor complexes consisting of a ligand binding component, neurophilin, and a signal transducing component, called plexin (Huber et al., 2003). Studies in flies and mice suggest that semaphorin primarily acts as short-range inhibitory cues that repel axons away from inappropriate regions. Interestingly there are studies suggesting that semaphorins may also act as attractive cues for certain axons. Class 3 semaphorins (Sema3s) are the most extensively studied vertebrate class. In vitro studies have revealed essential roles of class 3 semaphorins in eliciting growth cones responses as well as the understanding of signal transduction mechanisms that mediate these mechanisms. In addition, in vivo studies have implicated roles for Sema3 in the formation of several central and peripheral tracts as well as the axons of cranial, olfactory, motor, and hippocampal nerves (Cloutier et al., 2004; Falk et al., 2005; Huber et al., 2005; Kitsukawa et al., 1997; Liu and Halloran, 2005; Taniguchi et al., 1997; Wolman et al., 2004).

\subsection{1a4 Ephrins}

Ephs are a large group of receptor tyrosine kinases (RTKs) that bind to ephrins. Ephrin 'ligands' and Eph 'receptors' are membrane-bound resulting in signals that are localized to areas of cell contact (Lisabeth et al., 2013). After binding, both Eph- and 
ephrin-expressing cells are capable of transducing signals that lead to changes in cellular behavior. Thus, Ephs and ephrins signal bidirectionally, with ephrin induction of Eph signaling termed 'forward' signaling and Eph induction of ephrin signaling called 'reverse' signaling. Bidirectional signaling allows regulation of axon and target cell responses after an interaction. Eph-ephrin proteins were first identified as contact repellents, however, Eph-ephrin proteins can promote adhesion or repulsion depending on the signaling context (Arvanitis and Davy, 2008). Phenotypic analyses of Eph and ephrin mutant mice have shown that Eph-ephrin signaling is used to regulate cell-cell interactions in a variety of developmental processes, including cell migration (Chin-Sang et al., 1999; George et al., 1998; Smith et al., 1997) and axon guidance (Williams et al., 2003; Yokoyama et al., 2001).

\subsubsection{Ig superfamily (IgSF) CAMs in axon guidance and}

\section{fasciculation}

Navigation of axons depends on the interactions of growing axons with earlier grown axons of neighboring neurons which has already reached to its target. Axons adhere to either extracellular matrix or another cell surface by using specific cell surface receptors. Large number of IgSF-CAMs have been identified on growing axons, including NCAM1 (Cunningham et al., 1987), NCAM2 (Hamlin et al., 2004), L1CAM (Brittis et al., 1995), CHL1 (Schlatter et al., 2008), NRCAM (de la Rosa et al., 1990), CNTN1 (Falk et al., 2002), CNTN2 (Stoeckli and Landmesser, 1995; Suter et al., 1995), 
and ALCAM (Avci et al., 2004). Ig superfamily CAMs mediate intracellular communication and adhesion by interacting homophilically (i.e. between IgSF-CAMs of the identical types) and heterophilically (i.e. between different types of IgSF-CAMs) in cis (i.e. located in the plane of the plasma membrane) and trans (i.e. located in two contacting cell surfaces) conformations on neuronal surfaces. IgSF CAMs have been shown to play an important role in correct navigation and axon fasciculation of axons using a diverse array of in vitro techniques. Animals deficient for certain IgSF-CAMs exhibit significant errors in pathfinding of corticospinal, callosal, retinal, and hippocampal axons, suggesting that these molecules are critical factors in mediating pathfinding in vivo (Maness and Schachner, 2007). However, it is unclear how Ig superfamily CAMs function in vivo and how these deficits influence the development and function of the neural circuits. Therefore, in chapter 3,1 investigate the roles of Cntn2, a member of Ig superfamily CAM, in the development and function of neural circuits in zebrafish.

\subsection{Cell types examined to analyze axon guidance and}

\section{fasciculation}

\subsubsection{Interneurons of the nucleus of the medial longitudinal fascicle (nucMLF)}

The axons of the nucleus of the medial longitudinal fascicle (nucMLF) are the first axons to extend in the zebrafish midbrain (Hjorth and Key, 2002). nucMLF are bilateral 
clusters of neuronal cell bodies that lie in the ventral midbrain. nucMLF consists of a cluster of 6-8 neurons at the onset of MLF formation which occurs at 16 hpf. The cluster grows to approximately 30-35 neurons by $24 \mathrm{hpf}$ (Chitnis and Kuwada, 1990; Ross et al., 1992). Each nucMLF cells extend an axon posteriorly along the ipsilateral ventral neural tube. The caudal-most nucMLF cell extends the leading MLF axon into the hindbrain, and then the more rostrally positioned cells extend axons that fasciculate with the leading axon (Wolman et al., 2007) (Fig. 1.3A).

Using in vivo time-lapse imaging of $T g$ (pitx2c:gfp) transgenic line which expresses a green fluorescent protein (GFP) in nucMLF neurons (Fig. 1.3B), Wolman and colleagues showed that nucMLF axon guidance is controlled by both positive axonaxon interactions and guidance by surrounding tissue (Wolman et al., 2008). Morpholino studies have shown that guidance molecule, Contactin-2 (Cntn2), a member of Ig superfamily CAMs, guides the direction of axon extension by interpreting environmental cues and mediating positive axon-axon contacts. However, knockdown of $\mathrm{L} 1$, another Ig superfamily CAM expressed by nucMLF (Tongiorgi et al., 1995) leads to MLF defasciculation in the hindbrain but does not cause any defects of the nucMLF (Wolman et al., 2007). These data suggest that not all CAMs expressed by these neurons are required for MLF axon guidance. The first axons to extend in the brain use the extracellular matrix (ECM) as growth substrate and guidance sources. Laminins, a component of the ECM, are heterotrimeric glycoproteins, consisting of $\alpha, \beta$, and $y$ polypeptide chains which form a major component of the ECM in the central nervous system (Powell and Kleinman, 1997). A laminin-a1 mutant, bashful (Paulus and 
Halloran, 2006) also exhibits nucMLF defects as seen in cntn2 morphants. Loss of laminin- $\alpha 1$ causes nucMLF axons to extend into surrounding tissue in incorrect directions and reduces axonal growth rate. This has been shown to result in stunted nucMLF axons that fail to extend beyond the hindbrain (Wolman et al., 2008). However, unlike Cntn2, Laminin- $\alpha 1$ does not affect axon-axon interactions but does regulate neuronal polarity and influence growth cone responsiveness to surrounding cues (Wolman et al., 2008).

Optomotor response (OMR) is one of the innate sensory-driven locomotor behavior (Bilotta, 2000; Neuhauss et al., 1999). In this, larvae respond to the whole-field visual motion (Orger et al., 2000) by swimming and turning to maintain a stable position with respect to their visual environment (Portugues and Engert, 2009). nMLF is the most prominent group of neurons activated by visual stimulation that specifically elicits forward-directed locomotion (Orger et al., 2000). nucMLF is active in response to various stimuli including the spontaneous swimming and has been implicated in modulating locomotion in larval zebrafish (Sankrithi and O'Malley, 2010).

\subsubsection{Rohon-Beard (RB) sensory neurons}

Rohon-Beard (RB) neurons are multipolar primary sensory neurons present in the embryonic zebrafish spinal cord (Fig. 1.4A). They are located bilaterally in the dorsal spinal cord and have a cell body, two central axons, and a peripheral axon (Fig. 1.4B, C). RB neurons extend their central axons longitudinally within the dorsal spinal cord both rostrally and caudally and send their highly branched peripheral axons 
dorsolaterally from the spinal cord. Peripheral axon runs through segmental myotomes and innervates skin that transmits sensory stimuli to the CNS (Bernhardt et al., 1990; Metcalfe et al., 1990; Ribera and Nusslein-Volhard, 1998). The RB neurons undergo programmed cell death resulting in complete elimination from the spinal cord by 5 days postfertilization (dpf), and their function is replaced by dorsal root ganglia neurons (Reyes et al., 2004; Williams et al., 2000).

Zebrafish embryos react to tactile stimuli from about $22 \mathrm{hpf}$. Tactile stimuli in the head region are mediated by a subset of trigeminal ganglion cells. However, tactile stimuli to the trunk are mediated by RB cells (Metcalfe et al., 1990). Tactile stimulation activates RBs, which synapse onto primary ascending commissural interneurons (CoPAs). CoPAs which contact contralateral descending interneurons then activates motor neurons located contralaterally to the site of tactile stimulation (Carmean and Ribera, 2010).

Previous studies have shown that guidance molecules Semaphorin 3d (Sema3d)/Plexin A4 (PIxna4), and Slit2/Robo affects the outgrowth of peripheral axons, but not the central axon. Similarly, Contactin-2 only affects the outgrowth of central axons of RB neurons (Liu and Halloran, 2005; Miyashita et al., 2004) but not the peripheral axons. These findings suggest that the outgrowth of peripheral and central axons is regulated by different molecular machinery. 


\section{4.3 Retinal Ganglion Cell (RGC) neurons}

Visual information is carried from the eye to the brain via the axons of retinal ganglion cell axons. RGC axons navigate through a series of intermediate targets on their route to their destination. Guidance molecules like netrins, slits, and ephrins play a key role in the guidance of RGC axon (Deiner et al., 1997; Thompson et al., 2009; Williams et al., 2003).

Due to optical transparency of embryos, zebrafish have proven to be an excellent model for studying axon guidance in an in vivo context. RGC axons must make several decisions along their pathway and respond to various attractive or repulsive cues to reach their appropriate target. In zebrafish, the first RGCs are born at $28 \mathrm{hpf}$, and all axons cross the midline. Retinal axons navigate dorsally through the optic tract and reach the tectum at $48 \mathrm{hpf}$. Sema3d, a secreted semaphorin, is expressed at the midline and guides the crossing of RGC axons (Sakai and Halloran, 2006). Laminins are important components of the basal lamina and are known to be highly permissive as well as an instructive cue. Laminin-1 has been shown to be a permissive substrate for neurite outgrowth for different neural cell types (Powell and Kleinman, 1997). In vivo, laminin- $\alpha 1$ has been shown to play an important role in guiding RGC axons (Paulus and Halloran, 2006). Cntn2, a cell adhesion molecule of Ig superfamily, has been shown to be required for the fasciculation of RGC axons in mouse (Chatzopoulou et al., 2008). In zebrafish, Cntn2 is expressed on RGC neurons and axons (see Fig. 3.11E-F). In 
Chapter 3, we have examined the roles of Cntn2 in RGC axon guidance and fasciculation.

Since the roles mentioned above of Cntn2 in nUCMLF and RB neuron

development were implicated from morpholino studies, in Chapter 3, I have examined

the role of Cntn2 in development and function of nucMLF and Rohon-Beard neurons by

using null mutants.

\subsection{ADDITIONAL PROJECTS CONDUCTED DURING PHD}

\section{STUDIES}

\subsection{1: Novel Method for rapid cell ablation in zebrafish (in collaboration with Dr. Elizabeth Bryda)}

Our cell ablation system utilized the human CD59 receptor (hCD59), and the bacterial toxin intermedilysin (ILY). Our data involving transient RNA injection and stable transgenic line suggest that hCD59/ILY method can ablate cells rapidly in zebrafish and can obviate limitations involving relatively slow onset of cell ablation seen with current methodologies. I have described our findings in detail in Chapter 6.

\subsection{2: Establishment of a zebrafish model for Giant Axonal}

\section{Neuropathy (GAN) (in collaboration with Dr. Mark Hannink)}

Giant Axonal Neuropathy (GAN) is a rare neurodegenerative disorder affecting axons in the peripheral and central nervous system, accompanied by axonal swellings 
and axon loss (Bruno et al., 2004; Sharma et al., 2012). While GAN is caused by presumptive loss-of-function mutations in the human GAN gene (Tazir et al., 2009), the etiology of the disease is poorly understood. We aimed to establish a zebrafish model of GAN to gain insight into the cellular and biochemical mechanisms underlying the disease phenotype. GAN encodes a 468 amino-acid protein, Gigaxonin, a member of the BTB-Kelch superfamily involved in the ubiquitin-proteasome pathway. In mice, gan is expressed extensively in multiple tissues, including the brain and spinal cord (Bomont et al., 2000). Similarly, we found that zebrafish gan is expressed from $18 \mathrm{hpf}$ to $4 \mathrm{dpf}$, with high expression in the brain at $4 \mathrm{dpf}$.

To investigate roles of gan in disease, we generated loss-of-function mutations in gan using the CRISPR/Cas9 system. We have identified two alleles with frameshift mutations resulting in premature stop codons. Since sensory-motor functions are compromised in GAN patients, we examined two behaviors in zebrafish gan mutants involving sensorimotor circuits: touch-evoked escape response and food intake. Wild type and mutant larvae exhibited normal escape responses when touched in the trunk. In contrast, a significantly larger proportion of mutants responded when touched on the head, consistent with extensive gan expression in the hindbrain, where touch responses are processed. Feeding is a complex behavior requiring the coordination of sensory (vision, olfaction) and motor (jaw movement, locomotion) activities. At $7 \mathrm{dpf}$, mutant larvae exhibited a significant decrease in food intake compared to wild type siblings, consistent with gan expression in the hindbrain, where the motor neurons driving jaw movements are located. These data suggest that zebrafish gan mutants have defects in 
sensorimotor functions consistent with the defects seen in the mouse GAN model and in human patients, and may represent a good model for GAN in a simple vertebrate. 


\subsection{FIGURES AND LEGENDS}

Figure 1.1: Radially migrating neurons moving along radial glia processes in three repetitive steps

1. Extension of leading process

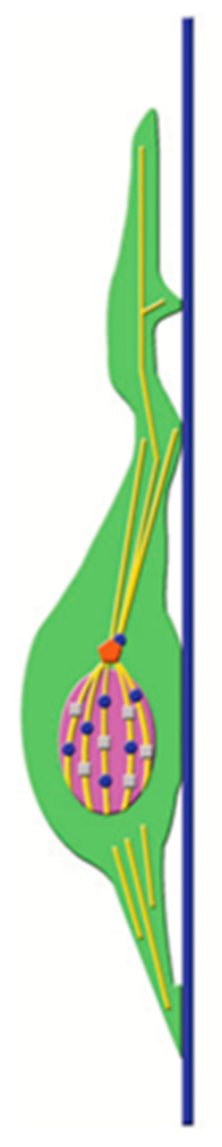

2. Nucleokinesis

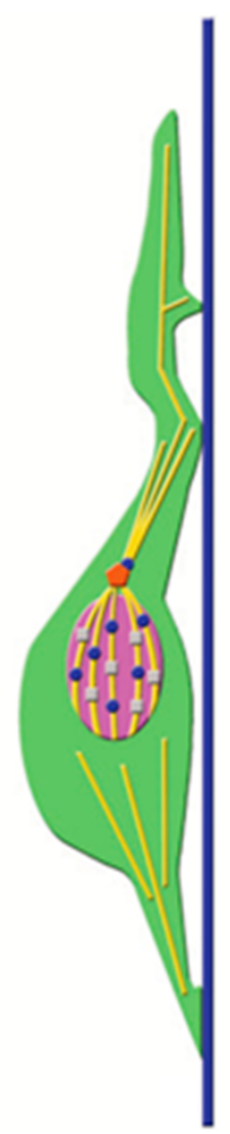

3. Retraction of trailing process

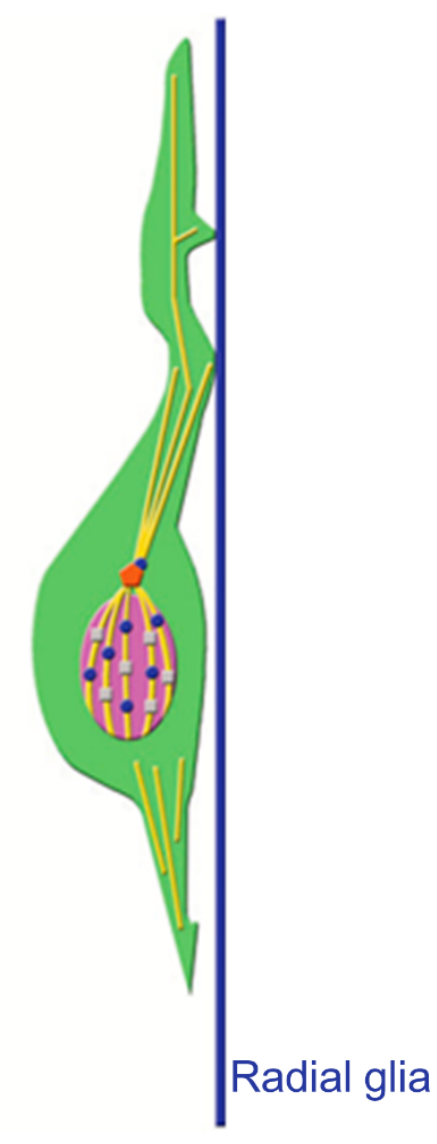


Figure 1.1: Radially migrating neurons moving along radial glia processes in three repetitive steps. (1) Migrating neurons first extend a leading process in the direction of migration. (2) This is followed nucleokinesis which causes forward movement. (3) In the final step, retraction of the trailing process takes place which enables the cell to move forward. Adapted from (Solecki et al., 2006). 
Figure 1.2: Facial branchiomotor (FBM) neuron migration in zebrafish

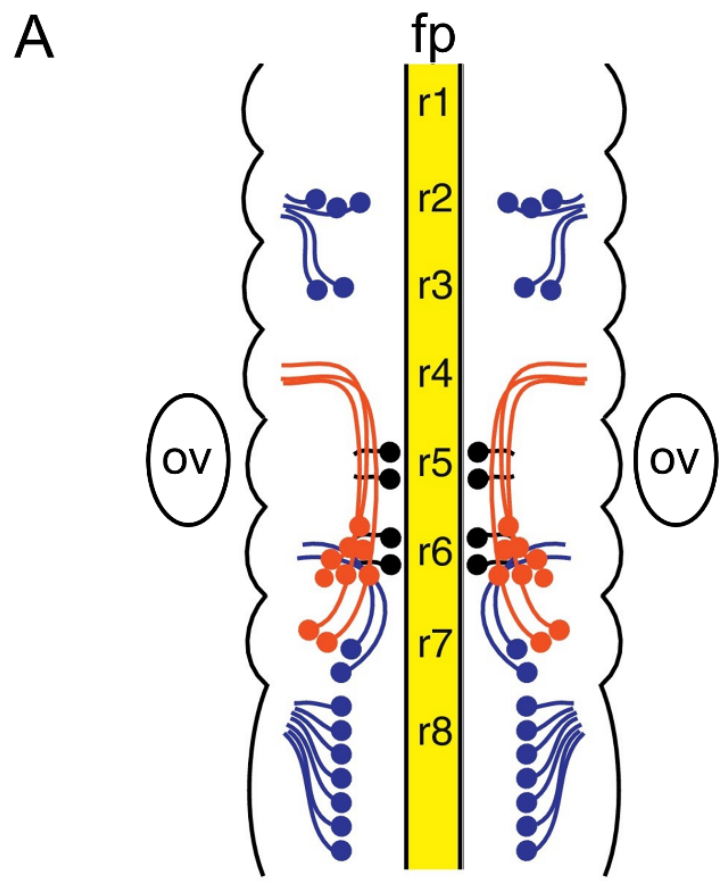

B

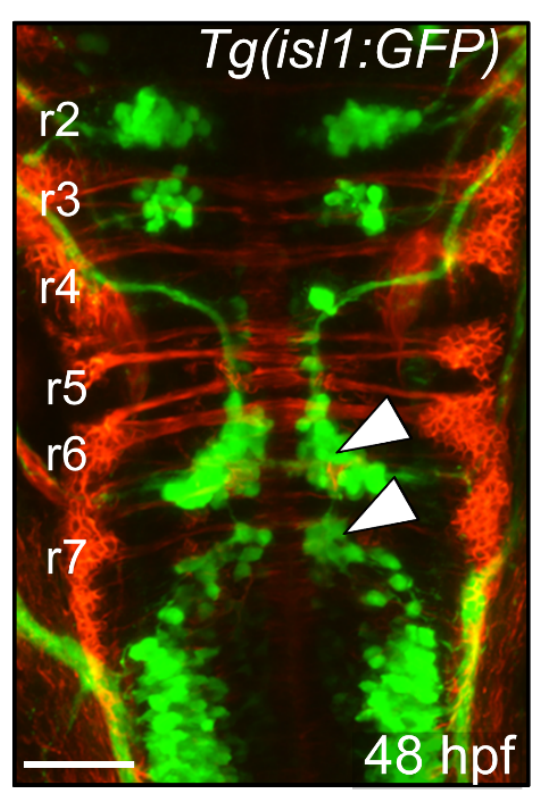


Figure 1.2: Facial branchiomotor (FBM) neuron migration in zebrafish. (A) Schematic rendering of $48 \mathrm{hpf}$ zebrafish BMN organization. FBM neurons in red, other BM neurons in blue, abducens motor neurons in black; ov, otic vesicle; fp, floor plate (yellow). (B) Tg(is/1:gfp) embryos were fixed at $48 \mathrm{hpf}$, and processed for immunohistochemistry with zn5 antibody (red) to label hindbrain commissural neurons and axons at rhombomere boundaries, and anti-GFP antibody (green) to label FBM neurons (arrowheads). Dorsal views of the hindbrain with anterior to the top. Scale bar in $\mathrm{B}=50 \mu \mathrm{m}$. Panel A modified from (Wanner and Prince, 2013). 
Figure 1.3: MLF axons extension and convergence

A

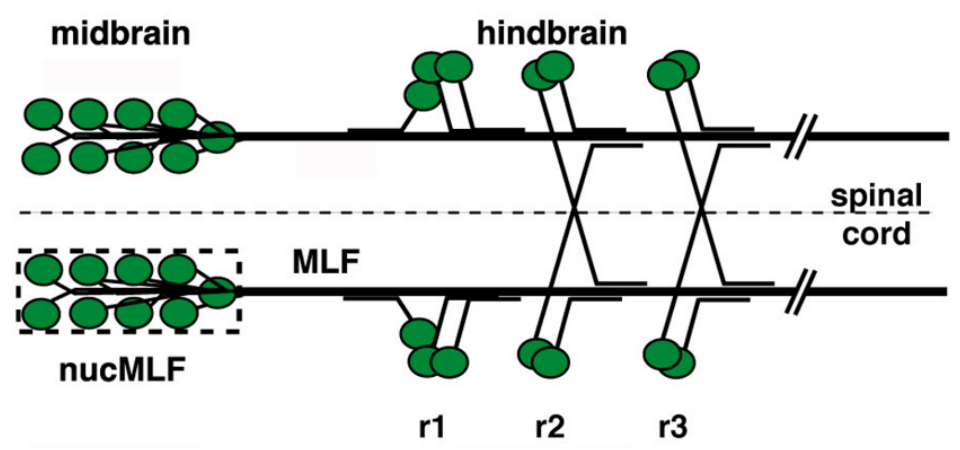

B

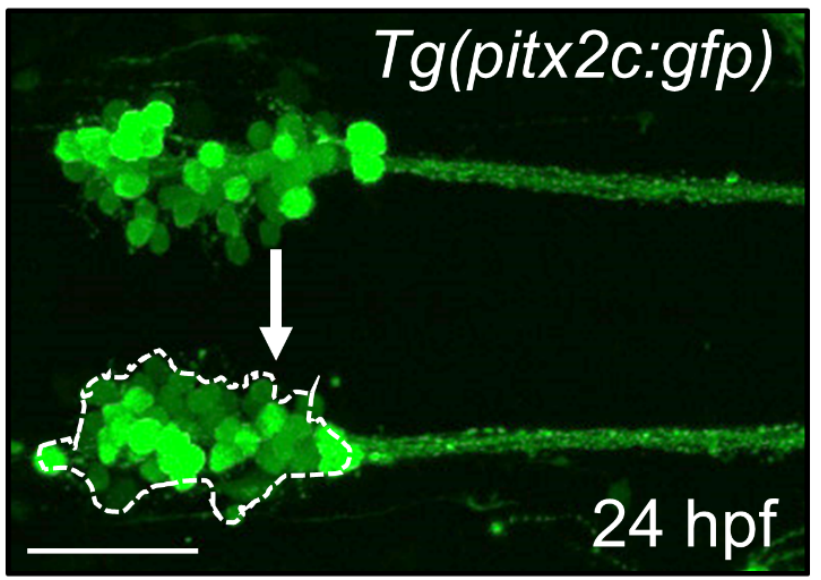


Figure 1.3: MLF axons extension and convergence. Ventral views with anterior to the left. (A) Schematic representation of MLF axons extending from midbrain to spinal cord. The dashed line denotes the ventral midline and dashed box surrounds the 'nucMLF zone'. r represents rhombomere. (B) Confocal projections of $T g$ (pitx2c:gfp) embryos labeled with anti-GFP antibody at $24 \mathrm{hpf}$. Ventral views of the midbrain with anterior to the left. Scale bar in $B=50 \mu \mathrm{m}$. Panel $A$ from (Wolman et al., 2008). 
Figure 1.4: Rohon-Beard neurons in zebrafish trunk
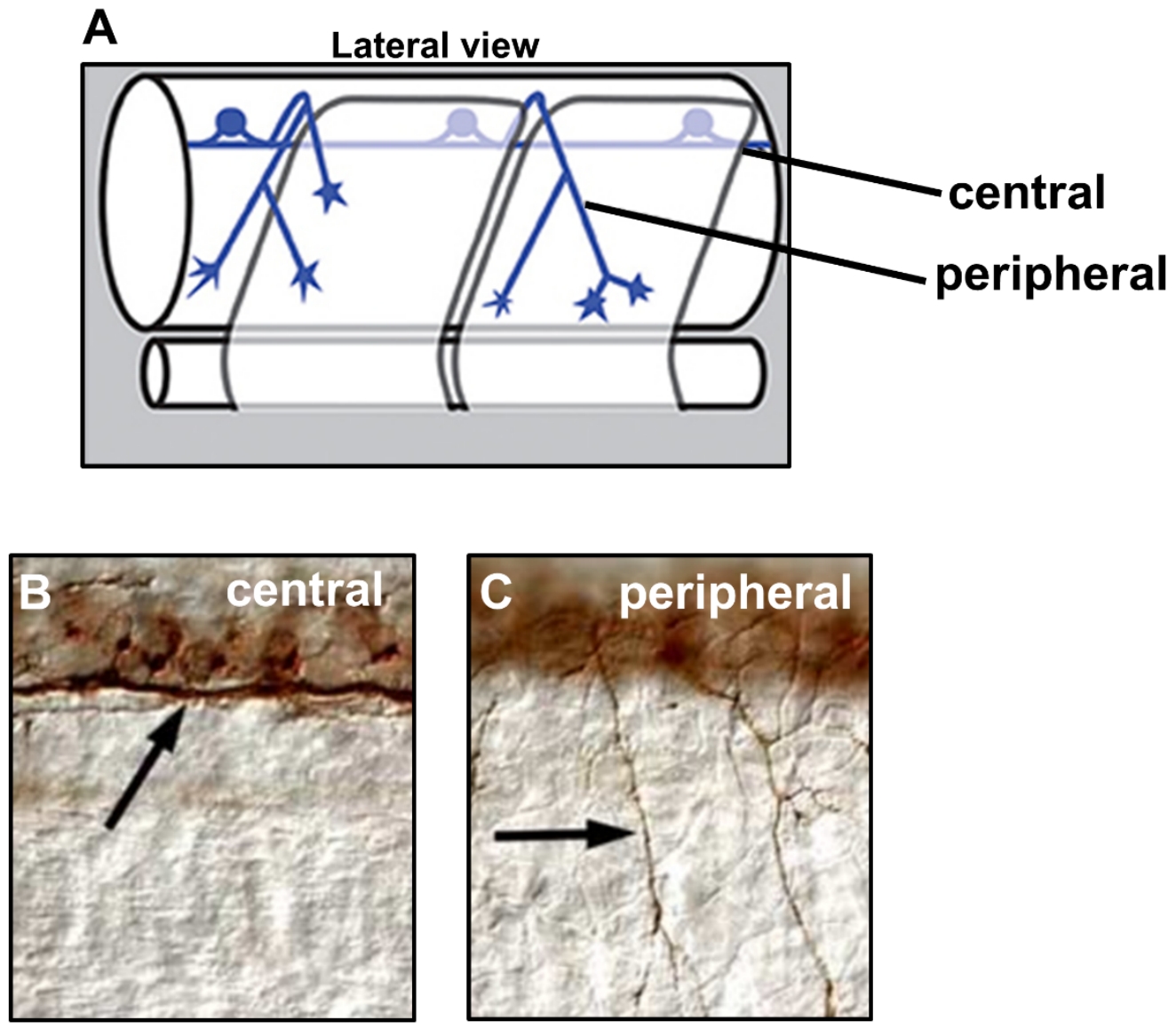
Figure 1.4: Rohon-Beard neurons in zebrafish trunk. Lateral views with anterior to the left. (A) Schematic of trunk showing Rohon-Beard axon pathways. (B, C) Zn-12 labeled $22 \mathrm{hpf}$ embryo showing central axons in spinal cord focal plane (B, arrow) and peripheral axons in the epidermal focal plane (C, arrow). Adapted from (Liu and Halloran, 2005). 


\section{CHAPTER 2: MATERIAL AND METHODS}

\subsection{FISH MAINTENANCE}

Zebrafish (Danio rerio) stock lines were maintained on a 14 hour light/ 10 hour dark cycle as described previously (Westerfield, 1995). Embryos were easily obtained by crossing adult fish. Depending on the nature of the experiment, embryos were obtained from pairwise crossing (1 pair) or population crossing (2-3 pairs). Embryos were grown at $28.5^{\circ} \mathrm{C}$ in embryo medium (E3) and staged by hours post fertilization (hpf) (Kimmel et al., 1995). The age of embryo was verified at somitogenesis stages. Embryos were treated with phenylthiourea (PTU, Sigma), a pharmacological pigmentation blocker, around $24 \mathrm{hpf}$ if prevention of pigmentation was desired. Embryos were fixed overnight at $4^{\circ} \mathrm{C}$ at appropriate ages in $4 \%$ paraformaldehyde in phosphatebuffered saline (PBS). Embryos were fixed for 2 hours at room temperature if they required light fixing.

\subsection{GENOTYPING EMBRYOS}

To genotype embryos, genomic DNA from individual embryos was extracted using $\mathrm{NaOH} / \mathrm{Tris}-\mathrm{HCl}$ (Meeker et al., 2007). Single embryo was then incubated in $50 \mu \mathrm{l}$ of $50 \mathrm{mM} \mathrm{NaOH}$ and boiled at $95^{\circ} \mathrm{C}$ for 10 minutes vortexting 2-3 times in between. Tubes containing genomic DNA were then cooled, and $5 \mu \mathrm{l}$ of $1 \mathrm{M}$ Tris- $\mathrm{HCl}(\mathrm{pH} 8)$ was added. Samples were then centrifuged at 13,000 RPM for 5 minutes at room temperature. $3 \mu$ of supernatant containing genomic DNA was used in a standard $25 \mu \mathrm{l}$ PCR reaction with appropriate primers. 


\subsection{GENOTYPING ADULTS}

To genotype adult fish, fish were briefly anesthetized using tricaine. Using a pair of fine scissors, the caudal fin was clipped halfway between the tip of the fin to a point at which fish scales end. Fish were returned to holding cages and allowed to revive before putting them in a circulating system. Fin-clipped fish were maintained in isolation in the aquarium for 2-3 weeks until the clipped fin was fully regenerated. The fin clip tissue was immersed in $0.5 \mathrm{ml}$ of lysis buffer and digested by rocking overnight at $55^{\circ} \mathrm{C}$. The following day, tubes with fin clip tissue were centrifuged for 20 minutes at room temperature. Clear supernatant containing genomic DNA was transferred to new $1.5 \mathrm{ml}$ centrifuge tubes containing $0.5 \mathrm{ml} 100 \%$ ethanol. The samples were then rocked for $2-3$ hours at room temperature until white/yellow strands were visible. Genomic DNA was then pelleted by centrifuging at $4^{\circ} \mathrm{C}$ for 20 minutes. The pellet was washed in cold $70 \%$ ethanol and the pellet was then resuspended in $20 \mu \mathrm{ldd} \mathrm{H}_{2} \mathrm{O} .2 \mu \mathrm{l}$ of this resuspension was used in a standard $25 \mu \mathrm{l} \mathrm{PCR}$ reaction with appropriate primers.

\subsection{MUTANT AND TRANSGENIC LINES}

\subsection{1 trilobite}

Trilobite allele $\left(\operatorname{tri}^{\text {tc240a }}\right)$ was identified based on their morphology during somitogenesis (Hammerschmidt et al., 1996; Solnica-Krezel et al., 1996). Through positional cloning, the gene encoded by trilobite was identified as vang/2 (Jessen et al., 2002). The trit ${ }^{\text {tc240a }}$ allele contains a 30 base pair insertion which results in the in-frame 
addition of 13 amino acids at Arg 21 (Jessen et al., 2002). Homozygous mutant larvae usually die by $6-7$ days of development, although about $10 \%$ of homozygotes escaped lethality and grow into fertile adults (Bingham et al., 2002). Homozygous mutants exhibit convergence extension defects as well as exhibit fully penetrant branchiomotor neuron defects.

\subsection{2 sleepy}

Sleepy allele (sly ${ }^{\text {ti263a }}$ ) was identified through large-scale zebrafish mutagenesis screens. This allele was identified based on defects in notochord differentiation resulting in shorter embryos with thin notochords (Odenthal et al., 1996). The sleepy locus maps to the lamc1 gene, which encodes laminin $\mathrm{Y} 1$. In addition to undersized retinas and displacement of the lens (Parsons et al., 2002), homozygous mutants also show defects in FBM neuron migration (Chandrasekhar, 2004).

\subsection{3 off-limits}

Off-limits allele (olt $\left.{ }^{r 689}\right)$ was identified from mutagenesis using $N$-ethyl- $N$-nitrosourea (ENU). Both zygotic, as well as maternal-zygotic olt embryos, display FBM neuron migration defects (Wada et al., 2006). The olt locus maps to zebrafish Frizzled3a and the olt ${ }^{w 689}$ carries a missense mutation which disrupts proper folding and thereby their function. 


\subsection{4 gpr125 $5^{\text {stl4o }}$}

Gpr125 is an adhesion G protein-coupled receptor shown to act as a modulator of the Wnt/planar cell polarity (PCP) signaling system (Li et al., 2013). gpr125 $5^{\text {stl40 }}$ allele was generated by Dr. Lilianna Solnica-Krezel's lab at Washington University. The allele was generated using TALENs which targeted GPCR proteolytic site. The gpr125 st/40 allele contains 19 base pair insertions resulting in early termination.

\subsection{5 cdh2 $2^{h i 3644}$}

Cadherin 2 allele $\left(c d h 2^{h i 3644}\right)$ came out the Hopkin's laboratory at MIT who generated and identified insertional mutations in 315 genes that resulted in visible morphological defects or lethality by 5 days post fertilization (Amsterdam et al., 2011; Golling et al., 2002). Cdh2 mutants exhibit defasciculation of optic nerves. However, retinal ganglion cell axons appropriately cross the midline (Barresi et al., 2010).

\subsection{6 dtrte370a}

Detour allele ( $\left.d t r^{t e 370 a}\right)$ was ENU-generated and originally identified based on its midline and retinotectal phenotypes (Brand et al., 1996; Karlstrom et al., 1996). The detour (dtr) locus maps to the gli1 gene. Cranial motor neurons are missing in $d t r^{\text {te370a }}$ embryos (Chandrasekhar et al., 1999).

\subsubsection{Islet1-GFP transgenic line $T g$ (is/1:gfp)}


The islet1-GFP transgenic line was generated using the promoter/enhancer of the islet (is/1) gene to drive GFP expression in cranial motor neurons (Higashijima et al., 2000). Is/1, a member of the Lim/homeobox gene family, is expressed in all post mitotic motor neurons early during the development (Inoue et al., 1994). $T g$ (is/1:gfp) fish express GFP in all branchiomotor neurons ( $\mathrm{nV}, \mathrm{nVII}, \mathrm{nX})$, except the $\mathrm{nIX}$ motor neurons. Unless otherwise stated, data presented here on FBM neuron migration analysis were performed in this transgenic line.

\subsubsection{Pitx2c-GFP transgenic line $T g(p i t x 2 c: g f p)$}

The Pitx2c:GFP transgenic line was generated using an internal promoter of the Pitx2 gene to drive GFP expression in trigeminal ganglia and the nucMLF neurons (Wolman et al., 2008). Unless otherwise stated, data presented here on nucMLF axon convergence analysis were performed in this transgenic line.

\subsection{GENOTYPING MUTANT LINES}

\subsection{1 gpr125 $5^{\text {st/40 }}$}

The following primers were used for genotyping:

Fwd: 5'-GATCTGCTGAGTCCGGTGAACGTGACGCTG- 3'

Rev: 5'-CACCATCAGCACGGCGTAGCTATTGAGTGA -3'

The PCR products were run on 2.5\% MetaPhor agarose (Lonza, Catalog \# 50181) gel for 2 hours at 35 volts to separate the bands. The gpr125 st/40 allele contains $19 \mathrm{bp}$ 
insertion, so the predicted fragment sizes are $195 \mathrm{bp}$ and $214 \mathrm{bp}$ for wildtype and mutant samples respectively. Heterozygotes show several bands due to heteroduplex formation between $195 \mathrm{bp}$ and $214 \mathrm{bp}$.

The PCR settings used were as follow:

$95^{\circ} \mathrm{C}: 2 \min$

$94^{\circ} \mathrm{C}: 40 \mathrm{sec}$

$68^{\circ} \mathrm{C}: 40 \mathrm{sec}$

$72^{\circ} \mathrm{C}: 40 \mathrm{sec}$

$72^{\circ} \mathrm{C}: 5 \min$

$10^{\circ} \mathrm{C}$ Hold

\subsection{2 cdh2 $2^{h i 3644}$}

The following primers were used for genotyping by PCR.
A: 5'- AACACGTCCTCAGAGTGCCAC - 3'
B: 5'- GTACGGTTACCAAGTCAATGTG - 3'
D: 5'- CTGTTCCATCTGTTCCTGAC - 3'
E: 5'- GTTCCTTGGGAGGGTCTCCTC - 3'

The PCR products were run on $1 \%$ agarose gel for 1 hours at 118 volts to separate the bands. The allele contains $\sim 3 \mathrm{~kb}$ insertion. Wildtype sample generates product at 603 bp with $A B$ primer set only. Heterozygous sample generates products at $603 \mathrm{bp}, 363$ bp, and 428 bp with primer sets $A B, A E$, and $B D$ respectively. Homozygous sample 
generates products at $363 \mathrm{bp}$, and $428 \mathrm{bp}$ with primer sets AE and BD respectively.

Homozygous sample does not produce any product with $A B$ primer set.

The PCR settings used were as follow:

$94^{\circ} \mathrm{C}: 2 \min$

$94^{\circ} \mathrm{C}: 40 \mathrm{sec}$

$60^{\circ} \mathrm{C}: 30 \mathrm{sec}$

$73^{\circ} \mathrm{C}: 60 \mathrm{sec}$

$73^{\circ} \mathrm{C}: 5 \mathrm{~min}$

$4^{\circ} \mathrm{C}$ Hold

\subsection{CRISPR/Cas9}

\subsubsection{Design and synthesis of guideRNA and Cas9 RNA}

The sgRNA design tool at http://crispr.mit.edu was used to identify possible target sites. Two criteria were employed while choosing sgRNA. First, since we were using T7 RNA polymerase in our synthesis, we only selected sgRNAs that began with $5^{\prime}$ G nucleotide. Second, we selected sgRNA target site with a restriction site for assaying the rate of mutagenesis in $\mathrm{F}_{0}$-injected embryos as well as genotyping putative founders. Two complementary oligonucleotides corresponding to the sgRNA target site were ligated to pT7-gRNA (Addgene 46759, (Jao et al., 2013). sgRNA was transcribed from BamH1 (Promega) linearized pT7-gRNA using MEGAshortscript T7 kit (Ambion). Following in vitro transcription reaction, guide RNA was purified using Micro Bio-Spin P- 
30 gel columns (Bio-Rad \#732-6250). Cas9 mRNA was synthesized using pT3TS-nlszCas9-nls (Addgene 46757, (Jao et al., 2013) plasmid. The plasmid was linearized using Xba1 (New England BioLabs, R0145S) and DNA was purified using QIAquick PCR purification kit (Qiagen). $1 \mu \mathrm{g}$ of linear DNA was used for in vitro transcription reaction (T3 mMESSAGE mMACHINE kit, Life Technologies, AM1338M). The mix of Cas9-encoding mRNA (150 pg/embryos) and sgRNA (50 pg/embryos) were injected into single cell $T g(i s / 1: g f p)$ embryos. Injected embryos were grown to adulthood to generate founder fish.

\subsubsection{Test of sgRNA cleavage efficiency}

To assay the rate of mutagenesis in $\mathrm{F}_{0}$-injected embryos, genomic DNA from individual injected embryos were extracted using $\mathrm{NaOH} / \mathrm{Tris}-\mathrm{HCl}$ (Meeker et al., 2007). Single $24 \mathrm{hpf}$ embryos were incubated in $50 \mu \mathrm{l}$ of $50 \mathrm{mM} \mathrm{NaOH}$ and boiled at $95^{\circ} \mathrm{C}$ for 10 minutes vortexing 2-3 times in between. Tubes containing genomic DNA were then cooled, and $5 \mu \mathrm{l}$ of $1 \mathrm{M}$ Tris- $\mathrm{HCl}(\mathrm{pH} 8)$ was added. Samples were then centrifuged at 13,000 RPM for 5 minutes at room temperature. $3 \mu$ of supernatant containing genomic DNA was used in a standard $25 \mu \mathrm{l}$ PCR reaction with primers spanning sgRNA target site. $15 \mu \mathrm{l}$ of PCR products were digested overnight at $37^{\circ} \mathrm{C}$ in a $30 \mu \mathrm{l}$ reaction containing unique restriction enzyme to the target site. Samples were then run on a $2.5 \%$ agarose gel. Successful mutagenesis is indicated by the loss of restriction site.

\subsubsection{Founder screening and F1 genotyping}


To screen for germline transmission of CRISPR-induced mutations, each putative founder fish was crossed with wild-type fish. At $24 \mathrm{hpf}$, about $10 \mathrm{~F} 1$ embryos were randomly screened for germline transmission from each founder fish following the procedure described above. Remaining F1 embryos from founder fish successfully transmitting mutations into germline were grown to adulthood for individual screening. Genomic DNA from individual F1 adult heterozygotes was extracted from tail tissue and genotyped following the procedure described for the injected embryos. PCR products from individual F1 heterozygotes exhibiting restriction site polymorphism were cloned into TOPO pcr2.1 vector using the TOPO TA cloning kit (Invitrogen). Plasmid DNA was isolated from individual transformants and sequenced using a T7 primer to identify the type of CRISPR-induced mutations.

\section{7 mRNA SYNTHESIS AND RNA GEL ELECTROPHORESIS}

Sense mRNA was synthesized using Ambion Message Machine kit (T7 or Sp6) following manufacturer's recommendations. Linear template $(1 \mu \mathrm{g})$ was incubated at $37^{\circ} \mathrm{C}$ with RNase-free water, $2 \mathrm{X}$ ribonucleotide mix, 10X transcription buffer and $10 \mathrm{X}$ RNA polymerase mix (T7 or SP6), generating a final reaction volume of $20 \mu \mathrm{l}$. The tube was then incubated for $2-4$ hours at $37^{\circ} \mathrm{C} .30 \mu$ of nuclease-free $\mathrm{ddH} 20$ and $30 \mu \mathrm{l}$ of Lithium Chloride was added, and mRNA was precipitated at $-20^{\circ} \mathrm{C}$ overnight. The next day, samples were microcentrifuged for 20 minutes at $4^{\circ} \mathrm{C}$. The pellet was washed with cold $70 \%$ ethanol and resuspended in $25 \mu \mathrm{l}$ of nuclease-free ddH20. mRNA was stored at $-20^{\circ} \mathrm{C}$. Yields and quality were determined by nano spectrophotometer and RNA gel 
electrophoresis. Formaldehyde agarose gels were used for visualizing the quality of synthesized RNA. 1 gram of agarose was melted in $84.8 \mathrm{ml}$ of ddH20. Under the fume hood, $5.2 \mathrm{ml}$ of room-temperature $37 \%$ formaldehyde was added and swirl gently. Next, $10 \mathrm{ml}$ of 10X MOPS (0.2 M MOPS, 0.05 sodium acetate, $0.01 \mathrm{M} \mathrm{EDTA,} \mathrm{pH} \mathrm{7.0,} \mathrm{stored}$ at $4^{\circ} \mathrm{C}$ ) was added and swirled gently before casting gel. 1X MOPS buffer was used as running buffer. RNA samples were prepared by mixing $2 \mu$ of RNA, $1 \mu$ of 1:10 diluted Ethidium bromide and $16 \mu$ of $1.25 x$ RNA loading buffer. RNA sample was then heated at $65^{\circ} \mathrm{C}$ for 10 minutes before loading in the gel. Smeared bands indicate a low-quality synthesis while single clean band typically indicate a successful transcription reaction.

\subsection{MORPHOLINO/RNA INJECTION}

Zebrafish embryos were injected at 1 to 2 cell stage. Embryos were lined up on glass slide glued to a Petri dish. Embryos were orientated with animal poles pointing towards the injection needle. Microcapillary needles (Stoelting Inc.) was pulled on a pipette puller, and the tip of the needle is broken using a micro-tweezer. Microcapillary needles were loaded with either mRNA or morpholino depending on the experiment and then installed into a micro-manipulator. The micro-manipulator was connected to a gaspressure pulse regulator that permits controlled pressure injections $(\sim 3-4 \mathrm{nl}$ per embryos). The injection volume was calibrated by measuring the diameter of droplets injected into mineral oil placed on a slide containing an etched micrometer scale. mRNA or morpholino either was injected into the blastomere or the boundary between blastomere and yolk. The needle was withdrawn slowly from the embryo and care was 
taken to do as little damage to the embryo as possible. The injected embryos are then rinsed off the slide with egg water into a Petri dish. The injected embryos were examined under the light microscope for mortality that might have occurred during injection. Dead embryos were removed to prevent contamination of remaining living embryos.

\subsection{IN SITU HYBRIDIZATION}

Embryos fixed in 4\% paraformaldehyde for at least 12 hours (typically overnight) at $4^{\circ} \mathrm{C}$ were dehydrated in $100 \%$ methanol (Fisher) for at least 5 hours (typically overnight) at $-20^{\circ} \mathrm{C}$. Embryos were rehydrated in a series of PBST $/ \mathrm{MeOH}$ washes as follows: 1 X5 minutes in 50\% MeOH/50\% PBST, 1 X5 minutes in 30\% MeOH/70\% PBST, then 2 X5 minutes in PBST. Following an hour room temperature fixation in 4\% paraformaldehyde, embryos were washed $3 \times 5$ minutes in PBST and then treated with proteinase K (Sigma; $23 \mathrm{mg} / \mathrm{ml}$ diluted to $10 \mu \mathrm{g} / \mu \mathrm{l}$ ) for 5 to 20 minutes depending on the age of the embryo. Embryos were washed fast $2 \mathrm{X}$ and washed $2 \mathrm{X} 5$ minutes in PBST. Embryos were then fixed for one hour at room temperature. After fixing, embryos were washed 3X5 minutes in PBST and 1X10 minute in 50\%PBST/50\% hybridization buffer and incubated in hybridization buffer for at least two hours at $70^{\circ} \mathrm{C}$. Embryos were incubated overnight in $\sim 100 \mathrm{ng}$ probe (1:100 dilution of the stock probe in hybridization buffer) at $65^{\circ} \mathrm{C}$. Following the overnight incubation, the probe was saved at $-20^{\circ} \mathrm{C}$ (probes can be re-used 3-5 times without affecting the efficiency of the signal produced). Embryos were then washed at $65^{\circ} \mathrm{C}$ for 1 hour in fresh hybridization buffer, 
then washed $2 \times 30$ minutes in wash $A, 1 \times 30$ minutes in wash $B$ and $2 \times 30$ minutes in wash $\mathrm{C}$, all at $65^{\circ} \mathrm{C}$. Embryos were then washed $1 \mathrm{X} 10$ minutes at room temperature in $50 \%$ wash C/50\% Maleic Acid Buffer $+0.1 \%(\mathrm{v} / \mathrm{v})$ Tween20, 2X10 minutes in Maleic Acid Buffer $+0.1 \%(\mathrm{v} / \mathrm{v})$ Tween20, and 1X10 minutes in blocking solution. Embryos were then incubated overnight in anti-digoxygenin antibody (diluted 1:5000 in blocking solution) after blocking for 3 hours at room temperature. Next, embryos were washed $8 X 15$ minutes in Maleic Acid Buffer $+0.1 \%(\mathrm{v} / \mathrm{v})$ Tween20, then 3X10 minutes in TMNT. For color development, embryos were incubated in the dark in TMNT containing $0.45 \%$ $(\mathrm{v} / \mathrm{v})$ NBT and $0.35 \%(\mathrm{v} / \mathrm{v}) \mathrm{BCIP}$ (Vector) for blue chromogenic reaction. The color reaction was stopped by washing embryos $3 \times 5$ minutes in $1 \mathrm{X}$ PBS. Embryos were fixed overnight in $4 \%$ paraformaldehyde at $4^{\circ} \mathrm{C}$, then were put through a glycerol series $(25 \%$, $50 \%$, then $70 \%$ glycerol) and mounted on $70 \%$ glycerol. Embryos can be stored in glycerol for extended periods before mounting if necessary.

\section{Solution recipes}

Hybridization Buffer $(30 \mathrm{ml})$

$\underline{\text { Final Concentration }}$

$15 \mathrm{ml}$ formamide (Fisher)

$50 \%$

$7.5 \mathrm{ml} 20 \mathrm{XSC}$

$5 X$

$30 \mu 50 \mathrm{mg} / \mathrm{ml}$ heparin (Fisher)

$50 \mu \mathrm{g} / \mathrm{ml}$

$1.5 \mathrm{ml} 10 \mathrm{mg} / \mathrm{ml}$ yeast tRNA (Roche) $500 \mu \mathrm{g} / \mathrm{ml}$

$30 \mu \mathrm{l}$ 100\% Tween-20 (Sigma)

$0.1 \%$

276 ㅅ 1M citric acid, pH 6.0 (LabChem, LC131180-1)

$9.2 \mathrm{mM}$ 
$5.664 \mathrm{ml} \mathrm{DEPC}$-treated ddH20 -

Vortex at highest setting after adding each component.

Store at $-20^{\circ} \mathrm{C}$.

$\underline{\text { Wash A }(10 \mathrm{ml})}$

$1 \mathrm{ml} 20 \times \mathrm{SSC}$

$5 \mathrm{ml}$ formamide

$10 \mu \mathrm{l} 100 \%$ Tween-20

$4 \mathrm{ml} \mathrm{ddH} 20$ -

Wash B $(10 \mathrm{ml})$

$1 \mathrm{ml} 20 \times \mathrm{SSC}$

$10 \mu \mathrm{l} 100 \%$ Tween-20

$9 \mathrm{ml} \mathrm{ddH} 20$ -

Wash C (10 ml)

$1 \mathrm{ml} 100 \%$ Wash B

9 l 100\% Tween-20

$9 \mathrm{ml} \mathrm{ddH} 20-$

Maleic Acid Buffer (1.6L)

$18.58 \mathrm{~g}$ Maleic Acid (Fisher)
Final Concentration

$2 X$

$50 \%$

$0.1 \%$

$\underline{\text { Final Concentration }}$

$2 X$

$0.1 \%$

$\underline{\text { Final Concentration }}$

$10 \%(0.2 \times$ SSC $)$

$0.09 \%$

$\underline{\text { Final Concentration }}$

$100 \mathrm{mM}$ 
$14.03 \mathrm{~g} \mathrm{NaCl}$

$9.6 \mathrm{~g} \mathrm{NaOH}$

Adjust $\mathrm{pH}$ to 7.5 with $10 \mathrm{~N} \mathrm{NaOH}$.

Bring to final volume with ddH20.

Sterilize by autoclaving for 20 minutes.

$\underline{10 \% \text { Stock Blocking Reagent }(100 \mathrm{ml})}$

$10 \mathrm{~g}$ Blocking Reagent (Roche, 1096176)

$100 \mathrm{ml}$ Maleic Acid Buffer

Dissolve granular blocking reagent in a microwave.

Sterilize by autoclaving for 15 minutes.

$\underline{2 \% \text { Blocking Solution }(10 \mathrm{ml})}$

$2 \mathrm{ml}$ 10\% Stock Blocking Reagent

$8 \mathrm{ml}$ Maleic Acid Buffer

$10 \mu \mathrm{l} 100 \%$ Tween-20

$\underline{\text { TMNT Buffer }(10 \mathrm{ml})}$

$8.23 \mathrm{ml} \mathrm{ddH} 20-$

$1 \mathrm{ml} 1 \mathrm{M}$ Tris $\bullet \mathrm{HCl}, \mathrm{pH} 9.5$

$500 \mu \mathrm{l} 1 \mathrm{M} \mathrm{MgCl} 2$

$250 \mu \mathrm{l} 4 \mathrm{M} \mathrm{NaCl}$
$150 \mathrm{mM}$

$150 \mathrm{mM}$

Final Concentration

$10 \%$

\section{$\underline{\text { Final Concentration }}$}

$2 \%$

$0.1 \%$

Final Concentration

$100 \mathrm{mM}$

$50 \mathrm{mM}$

$100 \mathrm{mM}$ 
$10 \mu \mathrm{l} 100 \%$ Tween-20

$0.1 \%$

$10 \mu \mathrm{l} 1 \mathrm{M}$ Levamisole

$1 \mathrm{mM}$

Do not autoclave. Use immediately.

\subsection{DAB IMMUNOHISTOCHEMISTRY}

Embryos were dechorionated and fixed in 4\% paraformaldehyde in PBS at room temperature for 3-4 hours. Embryos were washed in PBS with $0.1 \%$ tween 20 (PBST) $3 X 10$ minutes then blocked in incubation buffer (IB) (1\% sheep serum, $2 \mathrm{mg} / \mathrm{ml}$ Bovine Serum Albumin (BSA), 1\% DMSO, and 0.5\% Triton-X100 in PBS) for at least 1 hour in room temperature on a rotator. Embryos were incubated in primary antibody (diluted in incubation buffer) overnight at $4^{\circ} \mathrm{C}$. Next day, embryos were washed $2 \mathrm{Xfast}$, then $5 \times 10$ minutes with IB on a rotator. Embryos were incubated for 8 hours in secondary antibody $(2 \mu \mathrm{l}$ biotinylated universal antibody, $5 \mu \mathrm{l}$ horse serum, and $493 \mu \mathrm{l}$ IB) at room temperature. Embryos were then washed $2 X$ fast, then $5 X 10$ minutes with IB (no sheep serum or BSA) on a rotator. During the third wash, the tertiary solution was made.

Avidin-biotin color development $(\mathrm{A} / \mathrm{B})$ solution was mixed as follows: $1 \%(\mathrm{v} / \mathrm{v})$ reagent $\mathrm{A}$, $1 \%(\mathrm{v} / \mathrm{v})$ reagent $\mathrm{B}$ (Vectastain) in IB (no sheep serum or BSA) and allowed to sit for 30 minutes. Embryos were then incubated in the tertiary solution overnight at $4^{\circ} \mathrm{C}$. The next day, embryos were washed $2 X$ fast, then $5 \mathrm{X} 10$ minutes with PBST on a rotator.

Embryos were then transferred to 24-well plate where they were rinsed $3 \times 5$ minutes with PBS. The next day, embryos were incubated in $750 \mu$ diaminobenzidine (DAB) solution $(0.5 \mathrm{mg} / \mathrm{ml}$ in PBS) for $20-30$ minutes. While the embryos were incubating, start 


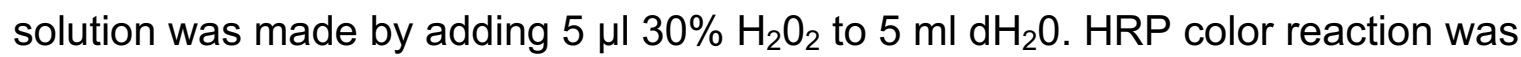
started by adding $75 \mu$ start solution to the embryos in the DAB solution. The color reaction was watched closely under dissecting scope. Once desired staining was achieved, the reaction was stopped replacing the DAB solution with stop solution (cold $0.2 \%$ azide in PBS). Embryos were washed $2 \times 5$ minutes in PBS and washed with $25 \%$, $50 \%$, and $70 \%$ glycerol in PBS. Finally, embryos were mounted in $70 \%$ glycerol.

Embryos can be stored in glycerol for extended periods before mounting if necessary.

\subsection{FLUORESCENT IMMUNOSTAINING}

Embryos were fixed in 4\% paraformaldehyde in PBS for at least 12 hours at $4^{\circ} \mathrm{C}$ or 6-8 hours at room temperature. Embryos were washed with IB (1 \% w/v BSA, 0.5\% $\mathrm{v} / \mathrm{v}$ Triton, and $1 \% \mathrm{v} / \mathrm{v}$ DMSO in PBS) $4 \times 30$ minutes then blocked once for 30 minutes in $\mathrm{IB}+1 \%$ horse serum (Sigma). Embryos were then incubated in primary antibody diluted in IB + horse serum overnight at $4^{\circ} \mathrm{C}$. Next day, embryos were washed $2 \times 30$ minutes with IB and blocked once for 30 minutes in IB $+1 \%$ horse serum. Embryos were then incubated in the dark (covered with aluminum foil) for 8-12 $\mathrm{h}$ in fluorescent secondary antibody diluted in 1:500 in IB+HS. Next day embryos were rinsed 3X5 minutes in PBS and fixed overnight in 4\% paraformaldehyde. After 4X5 minutes wash with PBS, embryos were put through a glycerol series $(25 \%, 50 \%$, then $70 \%$ glycerol) and mounted on $70 \%$ glycerol. It is important to store the embryos at $4^{\circ} \mathrm{C}$ in the dark to avoid fading of the fluorescent signals. Fluorescent signals are stable up to a week after placing in $70 \%$ glycerol. 


\subsection{ANTIBODY AND PROBE CONCENTRATION}

- Rabbit anti-GFP: 1:2000 (Invitrogen)

- Mouse anti-Zn8: 1:10 (Developmental Studies Hybridoma Bank)

- $\quad$ Mouse anti-Zn12: 1:250 (ZIRC)

- Rabbit anti-Cntn2: 1:500 (Kindly provided by Dr. Claudia Stuermer)

- Chicken anti-rabbit Alexa Flour 488: 1:500 (Invitrogen)

- Goat anti-mouse Alexa Flour 568: 1:500 (Life technologies)

- Donkey anti-rabbit Alexa Flour 568: 1:500 (Invitrogen)

- $\quad$ cntn2 ISH probe: 1:100

\subsection{QUANTIFICATION OF FBM NEURON}

Z-stack images (0.5 $\mu \mathrm{m}$ thickness, $150-200$ slices) were taken of dorsally-mounted stained embryos with Leica TCP SP8 MP confocal microscope at 40X magnification. Leica Application Suite X (LAS X) software was used to generate a 3D image of selected stacks of confocal images. FBM neurons at all dorsoventral levels were counted in the 3D image by examining each level.

\subsection{Dil LABELING OF AXONS AND PHOTOCONVERSION}

Embryos were fixed overnight at $4^{\circ} \mathrm{C}$ in $4 \%$ paraformaldehyde, washed in PBS, and embedded in $0.6 \%$ agarose (in Danieu buffer) on a glass slide. A $2 \mathrm{mg} / \mathrm{ml}$ solution of the lipophilic dye, 1,1'-Dioctadecyl-3,3,3',3'-Tetramethylindocarbocyanine Perchlorate (Dil, ThermoFisher Scientific) in dimethylformamide, was pressure injected into the 
structure of interest. Following overnight incubation at room temperature to allow for transport of Dil, the fluorescent signal was converted into a brown precipitate. Deyolked embryos were incubated in 3,3'-diaminobenzidine (DAB, Sigma; $0.5 \mathrm{mg} / \mathrm{ml}$ in $\mathrm{PO}_{4}$ buffer) for 15 minutes, mounted in DAB on a slide, and the fluorescence was photoconverted by examining the structures of interest under epifluorescence for $20-30$ minutes. Photoconverted embryos were post-fixed and mounted in $70 \%$ glycerol.

\subsection{WESTERN BLOTTING}

$10 \mathrm{ml}$ of lysis buffer was prepared fresh containing $1 \mathrm{ml}$ of $10 \mathrm{X}$ Cell Lysis Buffer (\#9803, Cell Signaling), $100 \mu \mathrm{l}$ of $0.1 \mathrm{M}$ PMSF, 1 complete protease inhibitor cocktail tablet (Roche), and $9 \mathrm{ml}$ distilled water. Protein was isolated using $200 \mu \mathrm{l}$ of lysis buffer per 100 decapitated $48 \mathrm{hpf}$ heads. Samples were homogenized on ice by mechanical grinding, then rotated at $4^{\circ} \mathrm{C}$ for 20 minutes at 13,000 and immediately stored at $-80^{\circ} \mathrm{C}$ at least overnight. Upon thawing, samples were centrifuged at $13,000 \mathrm{rpm}$ for 5 minutes to pellet insoluble material. $30 \mu \mathrm{l}$ of the supernatant from each sample was diluted 1:1 in 2X loading buffer (prepared from 4X NuPAGE LDS Sample Buffer, Invitrogen) with $20 \%$ $\beta$ - mercaptoethanol, and boiled for 5 minutes at $95^{\circ} \mathrm{C}$. Western blotting was performed using standard protocols (Pan et al., 2014).

\subsection{TOUCH EVOKED ESCAPE RESPONSE ASSAY}

Larvae at $2 \mathrm{dpf}$ were placed in Petri dishes in E3 medium and aligned to the center of the field of view (20 $\mathrm{mm}$ in diameter). The tactile stimulus was a single touch to the head or trunk of the larva with a dissection needle. Each larva was tested only 
once. The larval responses were binned into three categories: No response (no movement after touch), Weak Response (muted movement with larva remaining in the field of view), and Strong Response (rapid and vigorous movement with larva swimming out of the field of view).

\subsection{SWIMMING ANALYSIS}

For analysis of swimming behavior, $7 \mathrm{dpf}$ larvae were placed singly in individual wells of a 24-well plate. Swimming activity was monitored at room temperature (21$22^{\circ} \mathrm{C}$ ) using a DanioVision system and EthoVision XT 8.0 locomotion tracking software (Noldus). Larvae were allowed to acclimate in the DanioVision system for 30 minutes in the dark. Swimming activity was then monitored for an hour each in light and dark phases. Distance moved and moving duration parameters were analyzed using EthoVision. Larvae were processed for genotyping, and tracking data were pooled for analysis according to genotype. All of this analysis was performed at Georgia Southern University in Dr. Sittaramane's lab. 


\section{CHAPTER 3: DISTINCT ROLES FOR THE CELL ADHESION MOLECULE CONTACTIN2 IN THE DEVELOPMENT AND}

FUNCTION OF NEURAL CIRCUITS IN ZEBRAFISH

Suman Gurung ${ }^{1}$, Emilia Asante ${ }^{1}$, Devynn Hummel ${ }^{1}$, Ashley Williams ${ }^{2}$, Oren Feldman-

Schultz $^{3}$, Mary C. Halloran ${ }^{3}$, Vinoth Sittaramane ${ }^{2}$, and Anand Chandrasekhar ${ }^{1}$

1, Division of Biological Sciences and Bond Life Sciences Center, University of Missouri, Columbia, MO 65211, USA.

2, Department of Biology, Georgia Southern University, Statesboro, GA 30458, USA.

3, Department of Integrative Biology, Department of Neuroscience, University of Wisconsin, Madison, WI 53706, USA.

Corresponding Author:

Dr. Anand Chandrasekhar

Division of Biological Sciences

Room 340D Bond Life Sciences Center

1201 Rollins St

University of Missouri, Columbia, MO 65211-7310

Running Title: Distinct roles for Cntn2 adhesion molecule in zebrafish

Keywords: zebrafish; Neuronal Migration; Axon guidance; Facial Branchiomotor Neuron; nucMLF; Cell adhesion molecule; Cntn2; VangI2; Morpholino; CRISPR/Cas9 Submitted to Mechanisms of Development 


\subsection{ABSTRACT}

Contactin2 (Cntn2)/Transient Axonal Glycoprotein 1 (Tag1), a neural cell adhesion molecule, has established roles in neuronal migration and axon fasciculation in chick and mouse. In zebrafish, antisense morpholino-based studies have indicated roles for cntn2 in the migration of facial branchiomotor (FBM) neurons, the guidance of the axons of the nucleus of the medial longitudinal fascicle (nucMLF), and the outgrowth of Rohon-Beard (RB) central axons. To study functions of Cntn2 in later stages of neuronal development, we generated cntn2 mutant zebrafish using CRISPR-Cas9. Using a null mutant allele, we detected genetic interactions between cntn2 and the planar cell polarity gene vang/2, as shown previously with cntn 2 morphants, demonstrating a function for cntn2 during FBM neuron migration in a sensitized background of reduced planar cell polarity signaling. In addition, maternal-zygotic (MZ) cntn2 mutant larvae exhibited aberrant touch responses and swimming, suggestive of defects in sensorimotor circuits, consistent with studies in mice. However, the nucMLF axon convergence, FBM neuron migration, and RB outgrowth defects seen in morphants were not seen in the mutants, and we show here that they are likely offtarget effects of morpholinos. However, MLF axons exhibited local defasciculation in MZcntn2 mutants, consistent with a role for Cntn2 in axon fasciculation. These data demonstrate distinct roles for zebrafish cntn2 in neuronal migration and axon fasciculation, and in the function of sensorimotor circuits. 


\subsection{INTRODUCTION}

Neuronal migration and axon guidance are critical developmental processes that are essential for establishing functional neural circuits underlying complex cognitive and motor functions. During development of the central nervous system, many newborn neurons travel long distances away from the germinal zone to their final locations, whereupon they extend axons and dendrites to assemble functional neural networks. Precise neuronal migration and axon guidance are dependent upon cell-cell and cellsubstrate interactions (Chao et al., 2009; Marin et al., 2010), which are mediated by a number of membrane-associated molecules. Membrane proteins playing important roles in neurodevelopment include the cell adhesion molecules of the immunoglobulin superfamily (IgSF-CAMs), which are anchored to the membrane by a glycosylphosphatidyl inositol (GPI) linkage or a single transmembrane domain, have varying numbers of Ig-like extracellular domains, and are widely expressed during vertebrate neural development (Gennarini et al., 2016; Stoeckli, 2004; Stoeckli and Landmesser, 1995).

Contactin2 (Cntn2)/Transient Axonal Glycoprotein 1 (Tag1), a GPI-linked IgSFCAM, has six Ig-like domains and four fibronectin domains (Furley et al., 1990), and can bind homophilically (Kunz et al., 1998) as well as heterophilically with other IgSF CAMs such as L1, NgCAM, and NrCAM (Buchstaller et al., 1996; Fitzli et al., 2000; Kuhn et al., 1991; Kunz et al., 1998; Pavlou et al., 2002; Suter et al., 1995). Cntn2 can also interact with contactin-associated protein-like 2 (Caspr2) at the juxtaparanode region in neurons (Tzimourakas et al., 2007). Cntn2 is broadly expressed by various cortical, cranial 
nerve, and spinal neurons and axons (Dodd et al., 1988; Yamamoto et al., 1986). It has been demonstrated to play a role in the fasciculation and guidance of dorsal root ganglion axons in mice (Kunz et al., 1998; Law et al., 2008) and spinal commissural axons in chick (Stoeckli and Landmesser, 1995), in the migration of mitral cells in the mouse olfactory bulb (Bastakis et al., 2015), and in sensorimotor gating and coordination in mice (Savvaki et al., 2008).

In zebrafish, cntn2 is expressed in specific neuronal types during development (Liu and Halloran, 2005; Sittaramane et al., 2009; Warren et al., 1999; Wolman et al., 2008) (Fig. 3.1). Morpholino (MO)-mediated knockdown studies indicated that cntn2 is required for the organization of the midbrain nucleus of the medial longitudinal fascicle (nucMLF) and the convergence of nucMLF axons into the fascicle (Wolman et al., 2008), the growth and fasciculation of Rohon-Beard (RB) central axons in the spinal cord (Liu and Halloran, 2005), and the caudal migration of facial branchiomotor (FBM) neurons in the hindbrain (Sittaramane et al., 2009). Interestingly, cntn2 genetically interacts with the planar cell polarity gene vangl2, which encodes a four-pass transmembrane protein (Jessen et al., 2002), during FBM neuron migration (Sittaramane et al., 2009), suggesting that Cntn2 and Vangl2 participate in a common mechanism to regulate this process. While these MO-based data are consistent with Cntn2-associated functions in mouse and chick, potential later functions for zebrafish cntn2 in sensorimotor circuits and behavior could not be examined because of reduced efficacy of MO-mediated knockdown after 1-2 days of development (Bill et al., 2009). 
Moreover, although appropriate controls (Eisen and Smith, 2008) were performed, a caveat of MO-mediated knockdown experiments is the possibility of off-target effects.

Therefore, to investigate roles for zebrafish cntn2 at later developmental stages and to address potential concerns with the MO knockdown studies, we generated lossof-function mutations in cntn2 using CRISPR/Cas9. We generated two cntn2 mutant lines (cntn2 $2^{\text {zou20 }}$ and cntn2 $2^{\text {zou22 }}$ ) representing null alleles. Using the null mutants, we detected genetic interactions between cntn2 and vang/2, as shown previously with morphants, definitively demonstrating a function for cntn2 in FBM neuron migration. In addition, maternal-zygotic (MZ) cntn2 mutant larvae exhibited aberrant touch responses and swimming, suggestive of defects in sensorimotor circuits, consistent with studies in mice. However, the nucMLF axon convergence, FBM neuron migration defects, and RB outgrowth defects seen in cntn2 morphants were not seen in MZcntn2 mutants, and our data indicate that the morphant phenotypes are likely off-target effects of the morpholino. Interestingly, MLF axons exhibited local defasciculation in MZcntn2 mutants, consistent with a role for $\mathrm{Cntn} 2$ in axon fasciculation. These data demonstrate distinct developmental roles for zebrafish cntn2 in neuronal migration and axon fasciculation, and in the function of sensorimotor circuits.

\subsection{MATERIALS AND METHODS}

\subsubsection{Animals}


Zebrafish (Danio rerio) were maintained following standard protocols and University of Missouri ACUC guidelines as described previously (Sittaramane et al., 2013; Westerfield, 1995). Embryos were grown in E3 medium at $28.5^{\circ} \mathrm{C}$, staged by hours post fertilization (hpf) (Kimmel et al., 1995), and embryo age was verified at somitogenesis stages. $\operatorname{Tg}($ is/1:gfp) (Higashijima et al., 2000) and $\operatorname{Tg}($ pitx2c:gfp) (Wolman et al., 2008) fish were used to analyze FBM neuron migration, and nucMLF neurons and axons, respectively. The following two mutant lines were used: trilobite (tri ${ }^{\text {tc240a }}$, (Hammerschmidt et al., 1996)) and sleepy (sly ${ }^{\text {ti263a }}$, (Odenthal et al., 1996); kindly provided by Dr. Clarissa Henry, University of Maine, Orono) fish carrying mutations in the vang/2 and lamc1 genes, respectively. CRISPR-Cas9 cntn2 mutant lines were generated in the $T g(i s / 1: g f p)$ background.

\subsubsection{Generation of cntn2 null alleles using CRISPR-Cas9}

The sgRNA design tool at http://crispr.mit.edu was used to identify possible target sites. Two complementary oligonucleotides (5' TAGGCTAACAATGGTGCCGCAT-3'; 5'-AAACATGCGGCACCATTGTTAG-3') corresponding to the sgRNA target site in exon 4 (Fig. 3.2A) were used. sgRNA and Cas9 RNA were synthesized as described (Jao et al., 2013). The following primers were used for genotyping: Fwd: 5' -GACATATCATTTGCTGAAGAGTCG- 3'; Rev: 5' CTCGCGTTAGCAAGATACAAGTTA -3'. PCR products spanning the target site were digested overnight with Banl (NEB), and successful mutagenesis was indicated by the loss of the Banl site and the presence of an undigested PCR product (Fig. 3.2C). 
Injected embryos were grown to adulthood to generate founder fish, which were identified by outcrossing to wildtype fish and genotyping the F1 progeny for mutations in cntn2.

\subsubsection{Morpholino injections}

Antisense cntn2 morpholino (5' -CCACACCCAGACCAGACACTTATTT- 3';(Liu and Halloran, 2005; Sittaramane et al., 2009) and standard control morpholino (5' CCTCTTACCTCAGTTACAATTTATA- 3') were obtained from Gene Tools (Corvallis, OR). The cntn2 MO used in this study was previously shown to knockdown Cntn2 protein levels (Liu and Halloran, 2005), which we validated (Fig. 3.3).

\subsubsection{Immunohistochemistry and in situ hybridization}

Immunohistochemistry and in situ hybridization were performed using standard protocols (Sittaramane et al., 2009; Vanderlaan et al., 2005). The following primary antibodies were used: rabbit anti-GFP (Invitrogen, 1:2000 dilution), zn5/8 (Developmental Studies Hybridoma Bank (DSHB), 1:10 dilution), rabbit anti-Cntn2 (kindly provided by Dr. Claudia Stuermer, University of Konstanz (Lang et al., 2001); 1:500 dilution), zn-12 (ZIRC, 1:250 dilution). The following secondary antibodies were used: chicken anti-rabbit Alexa Fluor 488 (Invitrogen; 1:500 dilution), goat anti-mouse Alexa Flour 568 (Life technologies, 1:500 dilution), donkey anti-rabbit Alexa Flour 568 (Invitrogen, 1:500 dilution). Images were taken with an Olympus BX60 or Leica TCP 
SP8 MP confocal microscope, processed for brightness and contrast, and composed into Figures using Photoshop or Powerpoint software.

\subsubsection{Western blot}

Total protein was isolated from the heads of $2 \mathrm{dpf}$ larvae as previously described (Hoffman et al., 2016). Western blotting was performed using standard protocols (Pan et al., 2014). The following primary antibodies were used: rabbit anti-Cntn2 ((Lang et al., 2001); 1:500 dilution), anti-acetylated $\alpha$-tubulin (Sigma, 1:2000 dilution). The following secondary antibodies were used: goat anti-rabbit lgG, HRP conjugate (Santa Cruz Biotechnology, 1:5000 dilution) and HRP-goat anti-mouse IgG $(\mathrm{H}+\mathrm{L})$ (Invitrogen, 1:2500 dilution).

\subsubsection{Quantification of FBM neurons in the hindbrain}

Z-stack images (0.5 $\mu \mathrm{m}$ thickness, $150-200$ slices) were taken of dorsallymounted stained embryos with Leica TCP SP8 MP confocal microscope at 40X magnification. Leica Application Suite X (LAS X) software was used to generate a 3D image of selected stacks of confocal images. FBM neurons at all dorsoventral levels were counted in the 3D image by examining each level.

\subsubsection{Genotyping immunostained embryos}


The head fragments of antibody-stained embryos were mounted for quantification of FBM neuron migration, and the corresponding tail fragments were genotyped. Genomic region spanning the point mutation in the trilobite $\left(\operatorname{tri}^{\mathrm{t}}{ }^{240 a}\right)$ allele (Jessen et al., 2002) was PCR amplified using following primers: Fwd: 5'GCCTGGATGGTCACAGATTT-3'; Rev: 5' CCGGAAGTTTATCAGTATGGGAAACAC-3'. PCR product was purified using a PCR purification kit (Qiagen) according to the manufacturer's instructions and sequenced to detect the point mutation in each sample.

\subsubsection{Dil injections and photoconversion of Dil fluorescence}

Embryos were fixed overnight at $4^{\circ} \mathrm{C}$ in $4 \%$ paraformaldehyde, washed in PBS, and embedded in $0.6 \%$ agarose (in Danieu buffer) on a glass slide. A $2 \mathrm{mg} / \mathrm{ml}$ solution of the lipophilic dye, 1,1'-Dioctadecyl-3,3,3',3'-Tetramethylindocarbocyanine Perchlorate (Dil, ThermoFisher Scientific) in dimethylformamide, was pressure injected into the nasal retina, targeting the retinal ganglion cell layer. Following overnight incubation at room temperature to allow for transport of Dil, the fluorescent signal was converted into a brown precipitate as previously described (Chandrasekhar et al., 1997). Deyolked embryos were incubated in 3,3'-diaminobenzidine (DAB, Sigma; $0.5 \mathrm{mg} / \mathrm{ml}$ in $\mathrm{PO}_{4}$ buffer) for 15 min, mounted in DAB on a slide, and the fluorescence was photoconverted by exposing the labeled axons to epifluorescence for 20-30 min. Photoconverted embryos were post-fixed, and mounted in $70 \%$ glycerol. 


\subsubsection{Quantification of outgrowth of central axons of Rohon- Beard (RB) neurons}

Rohon-Beard neurons were sparsely labeled by injecting 12.5 pg of ngn1:GFPcaax DNA (Andersen et al., 2011) and 25 pg of Tol2 transposase RNA per embryo at the one-cell stage. Injected embryos were fixed at $18 \mathrm{hpf}$ for 4 hours at room temperature, and immunostained for GFP. RB neurons were binned into three groups based on the position of the cell body along the anterior-posterior (A-P) axis (1-5 somites, $6-10$ somites, $11-16$ somites). As a proxy to measuring the lengths of the central axons, the somite numbers corresponding to the tips of the anteriorly- and posteriorly-extending axons (ascending and descending axons, respectively) were recorded.

\subsubsection{Analysis of larval behaviors}

Larvae at $2 \mathrm{dpf}$ were placed in petri dishes in E3 medium, and aligned to the center of field of view (20 mm in diameter). The tactile stimulus was a single touch to the head or trunk of the larva with a dissection needle. Each larva was tested only once. The larval responses were binned into three categories (Fig. 3.9): No response (no movement after touch), Weak Response (muted movement with larva remaining in the field of view), and Strong Response (rapid and vigorous movement with larva swimming out of the field of view). 
For analysis of swimming behavior, $7 \mathrm{dpf}$ larvae were placed singly in individual wells of a 24-well plate. Swimming activity was monitored at room temperature (21$22^{\circ} \mathrm{C}$ ) using a DanioVision system and EthoVision XT 8.0 locomotion tracking software (Noldus). Larvae were allowed to acclimate in the DanioVision system for 30 minutes in the dark. Swimming activity was then monitored for an hour each in light and dark phases. Distance moved and moving duration parameters were analyzed using EthoVision. Larvae were processed for genotyping the cntn2 locus, and tracking data were pooled for analysis according to genotype.

\subsubsection{Statistical analysis}

We performed the chi-square tests using online software (www.quantpsy.org/calc.htm) to detect any significant differences in the distribution of: 1) FBM neuron migration phenotypes among groups used for genetic interaction studies, 2) touch-evoked escape responses for cntn2+/+ and MZcntn2 mutant embryos following a head touch or a trunk touch, and 3) MLF defasciculation phenotypes in cntn2+/+ and MZcntn2 mutants. We performed unpaired t-tests using GraphPad Prism 7 software to detect any significant differences in: 1) distance moved and moving duration between cntn2+/- and MZcntn2 mutant embryos, 2) RGC axon fascicle thickness in cntn2+/+, cntn2+/- and MZcntn2 mutant embryos, and 3) RB central axon length in cntn2+/+ and MZcntn2 mutant embryos.

\subsection{RESULTS}




\subsubsection{CRISPR-generated cntn2 alleles are null}

To examine roles for cntn2 in larval zebrafish, and to test the validity of the cntn2 morphant phenotypes, we generated loss-of-function mutations in cntn2 using CRISPRCas9 technology (Jao et al., 2013). We injected a single guide RNA (sgRNA) targeting exon 4, which encodes the second Ig domain of Cntn2 (Fig. 3.2A, B). We identified three independent alleles (cntn2 $2^{\text {zou20 }}, c n t n 2^{\text {zou21 }}$, and $\left.c n t n 2^{\text {zou22 }}\right)$, and established lines for the cntn2 $2^{z 0 u 20}$ and $c n t n 2^{z o u 22}$ alleles. The $c n t n 2^{z o u 20}$ allele has a 7 base pair (bp) insertion, and the cntn $2^{z o u 22}$ allele has an 11 bp deletion (Fig. 3.2A, C), which generate frameshifts and premature stop codons (Fig. 3.2B). Whole-mount in situ hybridization revealed that cntn2 expression is reduced in cntn2 $2^{\text {zou20 }}$ (Fig. 3.2D) and cntn2 ${ }^{\text {zou22 }}$ (data not shown) homozygous mutant embryos, consistent with nonsense-mediated decay (Chang et al., 2007). Importantly, a polyclonal antibody raised against the full-length Cntn2 protein (Lang et al., 2001) failed to detect any protein following immunohistochemistry (Fig. 3.2D) or western blotting (Fig. 3.2E) in both zygotic cntn $2^{\text {zou20 }}$ and cntn $2^{\text {zou22 }}$ homozygous mutants. These results strongly suggest that the CRISPR-generated cntn2 alleles are null. Interestingly, zygotic as well as maternalzygotic (MZ) cntn2 homozygous mutants are viable and fertile, and appear morphologically normal.

\subsubsection{FBM neurons migrate normally in cntn2 mutants}


In zebrafish, facial branchiomotor (FBM) neurons are born in rhombomere 4 (r4) and migrate posteriorly into r6 and r7 (Chandrasekhar et al., 1997; Higashijima et al., 2000) (Fig. 3.4A). cntn2 is expressed in FBM neurons in zebrafish and mouse (Garel et al., 2000; Sittaramane et al., 2009; Warren et al., 1999) (Fig. 3.1B). In cntn2 morpholino (MO)-injected embryos (morphants), FBM neurons exhibit migration defects, frequently failing to migrate out of $\mathrm{r} 4$ (Sittaramane et al., 2009). In cntn2 morphants (4-6 ng MO dose/embryo), FBM neurons migrated normally in $\sim 50 \%$ of the embryos, and either migrated poorly (Partial block) or largely failed to migrate (Severe block) in the remaining embryos (Fig. 3.4B, E). "Partial block" indicates reduced FBM neuron migration out of $\mathrm{r} 4$ on one or both sides, with FBM neurons found throughout the migratory pathway from $r 4$ to $r 7$, whereas "Severe block" indicates that a large majority of FBM neurons (estimated to be substantially greater than $50 \%$ ) remained in $\mathrm{r} 4$ on both sides, in the characteristic pattern seen previously in genetic mutants like trilobite (Bingham et al., 2002). To our surprise, FBM neurons migrated normally into r6 and $r 7$ in $100 \%$ of zygotic cntn2 homozygotes (Fig. 3.4C, E) of both alleles. The discrepancy in FBM migration phenotypes between cntn2 morphants and mutants may be explained by the presence of maternally contributed mRNA/protein in mutants but not in morphants. To test this possibility, we examined the migration of FBM neurons in maternal-zygotic (MZ) cntn2 mutants. FBM neurons migrated normally in 95\% of MZcntn2-/- embryos (Fig. 3.4D, E), strongly suggesting that cntn2 does not have an essential function during FBM neuron migration, and may play a subtle role at best. 


\subsubsection{Genetic interaction between cntn2 and vang/2 for FBM}

\section{neuron migration is preserved in mutants}

Vangl2, a four-pass transmembrane protein and a core component of Wnt/planar cell polarity pathway (Gray et al., 2011), is expressed ubiquitously in the hindbrain during the period of FBM neuron migration (Sittaramane et al., 2013; Sittaramane et al., 2009). We showed previously that cntn2 and vang/2 genetically interact during FBM neuron migration since injection of a suboptimal dose of cntn2 $\mathrm{MO}$ enhances the weak FBM neuron migration phenotype of vangl2 heterozygotes (Sittaramane et al., 2009). To test whether cntn2 mutants support this conclusion, we examined the offspring of MZcntn2-/- homozygous females and vang/2-/- homozygous mutant males, which are $100 \%$ double heterozygous embryos. FBM neuron migration defects were more severe and frequent in cntn2+/-; vang/2+/- double heterozygotes (Fig. 3.5D, E), compared to cntn2+/- or vang/2+/- embryos alone (Fig. 3.5B, C, E). FBM neurons migrated normally in $100 \%$ of cntn2+/- embryos (Fig. 3.5B, E), and failed to migrate out of $\mathrm{r} 4$ in only $5 \%$ of vang/2+/- embryos (Fig. 3.5C, E). By contrast, FBM neurons failed to migrate out of $r 4$ in $17 \%$ of cntn2+/-; vang/2+/- embryos (Fig. 3.5D, E). To further strengthen this subtle genetic interaction phenotype, we quantified FBM neuron migration in embryos obtained from a cross between vang/2+/- heterozygotes and cntn2-/- homozygous mutants, generating 50\% cntn2+/-; vang/2+/+ and 50\% double heterozygous (cntn2+/-; vang/2+/-) embryos. Since the genotypes of the embryos was unknown at the time of neuronal quantification, we selected ten immunostained embryos from each of three phenotypic 
groups corresponding to normal migration, partial and severe migration block (Fig. 3.5F$\mathrm{H}$ ), counted the number and examined the distribution of motor neurons in $r 4, r 5$, and (r6+r7) (Fig. 3.5I). Nearly $70 \%$ of FBM neurons failed to migrate out of $r 4$ in "severe block" embryos, while $87 \%$ and $65 \%$ of neurons migrated out of $r 4$ in "normal migration" and "partial block" embryos, respectively, providing high confidence in our phenotypic classes (Fig. 3.5E). Importantly, all embryos in the partial $(n=10)$ and severe block $(n=10)$ categories were subsequently genotyped as double heterozygotes, while most of the "normal migration" embryos (8/10) were genotyped as cntn2+/-; vang/2+/+. Taken together, the distribution of FBM migration phenotypes generated in two different genetic crosses supports the likelihood of genetic interactions between cntn2 and vang/2. This genetic requirement for cntn2, defined using a null allele, is revealed only in a sensitized genetic background of reduced planar cell polarity signaling, suggesting that cntn2 plays a definitive but minor role during FBM migration.

To further explore a role for cntn2 in FBM neuron migration, we examined another sensitized genetic background known to affect FBM neuron migration. We showed previously using suboptimal cntn2 $\mathrm{MO}$ doses and the bashful mutant that cntn2 genetically interacts with laminina1 (lama1), which encodes a broadly expressed Laminin subunit Laminina1 (Sittaramane et al., 2009). Since FBM neurons exhibit similar migration defects in bashful (lama1-/-) and sleepy (lamc1-/-) mutants (Chandrasekhar, 2004), which affect different Laminin subunits, we tested for genetic interactions between cntn2 and lamc1. We examined the offspring of MZcntn2-/homozygous females and lamc1+/- heterozygous males, $50 \%$ of which are double 
heterozygous embryos. We found no embryos with severe FBM neuron migration defects, and the fraction of embryos with partial defects was comparable to that seen in cntn2+/- and lamc1+/- populations (data not shown). These data indicate that cntn2 and lamc1 do not interact genetically for FBM neuron migration, and suggest that some morphant-associated phenotypes may not reflect true functions of cntn2.

\subsection{4 cntn2 mutants lack nucMLF axon convergence defects seen in morphants but exhibit MLF defasciculation}

Given the discrepancy between cntn2 morphant and mutant FBM neuron migration phenotypes, we examined other phenotypes previously seen in morphants, such as the nucMLF axon convergence defects (Wolman et al., 2008). First, we confirmed the morphant phenotype by injecting cntn2 MO into Tg(pitx2c:gfp) embryos, which express GFP in nucMLF cell bodies and axons (Wolman et al., 2008). In control MO-injected embryos, the nucMLF was found as bilateral groups of tightly clustered cells, which extended tight axon fascicles posteriorly (Fig. 3.6A, E). However, in cntn2 MO-injected embryos, the nucMLF cells were loosely packed, their axons were defasciculated and they failed to converge normally (Fig. 3.6B, E). In contrast to the morphants, we did not find any nucMLF axon convergence defects in either zygotic (Zcntn2-/-) or maternal-zygotic (MZcntn2-/-) embryos (Fig. 3.6C-E), consistent with the difference between morphant- and mutant-associated FBM neuron phenotypes (Fig. 3.4). 
We also examined the ability of MLF axons to maintain fasciculation in the absence of Cntn2. MLF axons remained tightly fasciculated as they extended through the midbrain and anterior hindbrain (Fig. 3.6F). Interestingly, MZcntn2-/- embryos showed defasciculation of MLF axons in these regions (Fig. 3.6G-I). Because the MLF axons are often loosely fasciculated posterior to $r 2$, we restricted our analysis to the area anterior to $\mathrm{r} 2$ (delineated by dotted line in Fig. 3.6F, corresponding to the hindbrain entry point for trigeminal sensory axons). We categorized embryos in blinded fashion into three groups based on the severity of the defasciculation phenotype: None (no defasciculation), Weak (small splits in the fascicle or very short stretches of defasciculated axons on one or both sides (Fig. 3.6G), and Strong (extensive stretches of split fascicles or axon defasciculation on one or both sides (Fig. 3.6H, I)). About $\sim 85 \%$ of MZcntn2 mutant embryos ( $n=32$ ) exhibited strong defasciculation, and $<5 \%$ showed no defects (Fig. 3.6J). In marked contrast, only $\sim 28 \%$ of $c n t n 2+/+$ embryos ( $n=32$ ) exhibited strong defasciculation, and $\sim 50 \%$ showed no defasciculation (Fig. 3.6J). These data suggest strongly that Cntn2 is involved in maintaining adhesions between MLF axons in the zebrafish midbrain and anterior hindbrain.

\subsubsection{FBM neuron and nucMLF defects in cntn2 morphants}

\section{are likely off-target effects}

The difference between cntn2 morphant and mutant phenotypes can be explained either by off-target effects of the morpholino (MO) (Eisen and Smith, 2008; 
Kok et al., 2015) or by genetic compensation from related genes in mutants but not in morphants (Rossi et al., 2015). To distinguish between these alternatives, we examined the effects of injecting cntn2 MO into cntn2+/+ and MZcntn2-/- embryos. If cntn2 MO injection causes defects in cntn2+/+ embryos but not in MZcntn2-/- embryos, then it would suggest genetic compensation (Fig. 3.7A). However, defects in both cntn2+/+ and MZcntn2-/- embryos following cntn2 MO injection would imply an off-target effect of the $\mathrm{MO}$ (Fig. 3.7A). As expected, FBM neuron migration and the development of nucMLF neurons and axons occurred normally in control MO-injected cntn2+/+ and MZcntn2-/- embryos (Fig. 3.7B-E, J, K). Consistent with previous studies (Sittaramane et al., 2009), migration of FBM neurons was strongly affected in cntn2+/+ embryos injected with cntn2 MO (Fig. 3.7F, J). Similarly, the organization and axonogenesis of nucMLF neurons was greatly perturbed in cntn2+/+ embryos injected with cntn2 MO (Fig. 3.7G, K), as shown previously (Wolman et al., 2008). Surprisingly, MZcntn2-/embryos, which lack functional cntn2 mRNA and contain no detectable Cntn2 protein, also exhibited FBM neuron migration and nucMLF convergence phenotypes following cntn2 $\mathrm{MO}$ injection (Fig. 3.7H-K). These data demonstrate that the FBM and nucMLF neuronal defects seen in cntn2 morphants are likely due to off-target effects of the MO.

\subsection{6 cntn2 mutants exhibit a defective escape response and have swimming deficits}


While some of the cntn2 morphant phenotypes appear to be off-target effects, the definitive phenotypes seen in MZcntn2 mutants (cntn2-vang/2 genetic interaction for FBM neuron migration and MLF defasciculation) motivated us to look for phenotypes in other cntn2-expressing cell types. In cntn2 morphant embryos, Rohon-Beard (RB) central axons grew more slowly and showed some defasciculation compared to control embryos (Liu and Halloran, 2005). To test whether the growth of RB central axons was affected in cntn2 mutants, we compared the lengths of RB central axons in equivalentstage cntn2+/+ and MZcntn2-/- embryos. RB neurons were sparsely labeled using a ngn1:GFP-caax Tol2 construct (Andersen et al., 2011). There was no reduction in RB central axon lengths in MZcntn2 mutants (Fig. 3.8), suggesting that the loss of Cntn2 does not affect RB central axon growth rates as seen in morphants. In addition, we did not observe obvious defasciculation of RB central axons in zn-12 antibody-stained MZcntn2-/- embryos at 24 hpf (data not shown). However, because the RB central axon defasciculation effect was variable in the morphants (Liu and Halloran, 2005), we reasoned that a behavioral test such as the touch-evoked escape response (Granato et al., 1996) would be a more sensitive assay for putative functional defects associated with these neurons. The escape responses of $2 \mathrm{dpf}$ larvae to head or trunk touch were binned (blinded to genotype) into three categories: No response, Weak response, and Strong response (see Materials and Methods). Larvae of both genotypes (cntn2+/+ and MZcntn2-/-) behaved similarly when touched on the head (Fig. 3.9A). However, larger numbers of MZcntn2-/- mutant larvae than cntn2+/+ larvae exhibited weak responses when touched in the trunk (Fig. 3.9B). Touch-evoked escape response defects in the 
trunk but not in the head suggest that MZcntn2 mutants may have a defect associated specifically with the RB neuron-mediated spinal sensorimotor circuit. Importantly, outgrowth of spinal motor axons was not affected in mutants (Fig. 3.10) consistent with a role for cntn2 in assembling the sensory component of the escape response circuit. While there was no clear defect in RB central axon outgrowth in mutants, we cannot rule out defects in other aspects of RB morphology or differentiation.

To further examine the effects of Cntn2 protein loss on the function of sensorimotor circuits, we analyzed swimming activity using the DanioVision system. Since Cntn2 is expressed in a subset of retinal ganglion cells (RGCs) and their axons (Fig. 3.1E-F), we examined light-evoked behaviors by measuring the swimming activity of $7 \mathrm{dpf}$ cntn2+/- and MZcntn2-/- larvae during "Lights off" and "Lights on" phases (60 minutes each) (Fig. 3.11A). Larval genotypes were determined after swimming activity measurements (See Materials and Methods). Total swimming distance and swimming duration were significantly lower in MZcntn2-/- mutants compared to cntn2+/- siblings during both phases (Fig. 3.11B, C). Importantly, total distance moved and activity during the "Lights off" phase were significantly lower than during the "Lights on" phase in both cntn2+/- and MZcntn2-/- larvae (Fig. 3.11B, C), indicating that light-dependent movement is conserved, and suggesting that the swimming deficits of MZcntn2 mutants result from circuit defects downstream of the visual pathway. We tested directly for visual pathway defects by examining the morphology of RGC axons following anterograde labeling with lipophilic dye Dil and photoconversion (see Materials and Methods). Since RGC axon fascicles were thinner in MZcntn2 mutants when compared 
to cntn2+/+ cousins, but not when compared to cntn2+/- siblings (Fig. 3.12), potential defects in the visual system cannot be ruled out. Taken together, the escape response and swimming activity phenotypes suggest that MZcntn2 mutants have deficits in sensorimotor circuitry, consistent with cntn2 expression in cell types (RB and RGC neurons) associated with these circuits.

\subsection{DISCUSSION}

A large number of membrane-associated molecules have been implicated in the guidance of migrating neurons and projecting axons during nervous system development (Maness and Schachner, 2007). With the advent and ease of application of CRISPR/Cas9 technology, it is now possible to readily test the biological role of any gene in zebrafish (Hwang et al., 2013). Here, we used CRISPR/Cas9 to generate a null mutant for a neural cell adhesion molecule Cntn2. Our data demonstrate that while some of the previously-described morphant phenotypes may result from off-target effects, cntn2 also plays definitive roles in assembling neural circuits in zebrafish.

\subsubsection{Comparison of cntn2 morphant and mutant phenotypes}

Morpholino-mediated knockdown experiments previously suggested roles for cntn2 in the caudal migration of FBM neurons (Sittaramane et al., 2009), the guidance of axons from the nucMLF (Wolman et al., 2008), and the growth of RB central axons (Liu and Halloran, 2005). However, MZcntn2 mutants did not phenocopy most of these morphant phenotypes. There are several explanations for the differences between the 
morphant and mutant phenotypes. First, the mutant might make partially functional Cntn2 protein due to translation in-frame from start codons downstream of the mutation in exon 4 . Second, the use of alternative, cryptic splice sites may skip exon 4 and yet generate truncated but functional Cntn2 protein. However, these outcomes appear unlikely since no Cntn2 protein is detected in MZ mutants (both alleles) using a polyclonal antibody generated using full-length Cntn2 protein (Lang et al., 2001). Such an antibody would likely recognize epitopes distributed throughout the length of the protein, and therefore detect truncated protein generated by alternative splicing or translational start sites. Exhaustive analysis of the genome assembly (GRCz11, 2017) did not identify any closely related genes (>75\% similarity in Immunoglobulin and Fibronectin domains), suggesting that the absence of a strong mutant phenotype is not due to genetic redundancy.

Although a few studies have shown that morphants and mutants for some genes have similar or overlapping phenotypes (Bill et al., 2009; Phillips et al., 2011), there are other cases where the two phenotypes do not match (Lebedeva et al., 2017; Moore et al., 2016). An analysis of several genes affecting development and organogenesis showed that $80 \%$ of the morphant phenotypes were not seen in mutants, and concluded that morpholinos produce a high false-positive rate (Kok et al., 2015). Two models can explain the discrepancy in phenotypes between morphants and mutants. In the first model, MOs can generate off-target effects by specifically knocking down the expression of another unidentified gene, but they can also be toxic and generate nonspecific phenotypes. In the second model, genetic compensation from related genes 
may occur in mutants, but not in morphants, resulting in the absence of a loss-offunction phenotype (Rossi et al., 2015). For instance, in egfl7 mutants, vascular defects generated in egfl7 morphants were not seen. However, several members of the emilin gene family were upregulated in mutants, potentially compensating for the loss of egfl7 (Rossi et al., 2015). It is possible that genomic disturbances caused by deleterious mutations activate compensatory pathways in mutants, while MOs, which only block the translation of mRNA, fail to do so.

In light of these studies, one explanation for the difference between the cntn2 morphant and mutant phenotypes is that other Cntn-like or other Ig superfamily CAM genes may be overexpressed and compensate for loss of cntn2 function in mutants but not morphants. The genetic compensation and off-target effect models were distinguished by examining the effect of injecting $\mathrm{MO}$ into the mutants (Fig. 3.7A; (Stainier et al., 2017)). Since the cntn2 morphant phenotypes for FBM and nucMLF neurons were readily seen in null mutants injected with cntn2 $\mathrm{MO}$, it is unlikely that these phenotypes are due to loss of Cntn2 function, but rather are due to off-target effects. Nevertheless, we have also documented cntn2 mutant-specific phenotypes associated with the FBM and nucMLF neurons, indicating that $c n t n 2$ plays definitive roles in the development of these neuronal populations.

\subsubsection{Role for Cntn2 in FBM neuron migration}

During nervous system development, migrating neurons respond to several cues in the environment, and interact with neighboring cells (Marin et al., 2010). Vangl2, a 
core component of Wnt/Planar Cell Polarity (PCP) pathway (Gray et al., 2011), is expressed ubiquitously in the hindbrain, and functions both within the FBM neurons and in the surrounding neuroepithelial cells for FBM neuron migration (Davey et al., 2016; Jessen et al., 2002; Sittaramane et al., 2013; Sittaramane et al., 2009). cntn2 is expressed in branchiomotor neurons, but not in the surrounding neuroepithelial cells, during FBM neuron migration (Sittaramane et al., 2009; Warren et al., 1999), which indicates that cntn2 likely functions in a cell-autonomous fashion. Laminina1 (lama1), which encodes a subunit of the extracellular matrix protein Laminin1, is expressed broadly (Sittaramane et al., 2009) and plays a role in FBM neuron migration (Paulus and Halloran, 2006). Using a combination of mutants and MOs, we previously showed genetic interactions between cntn2 and vang/2, and between cntn2 and lama1 (Sittaramane et al., 2009). Here, using only mutants, we again observed genetic interactions between cntn2 and vang/2, but not between cntn2 and lamc1, which encodes a different obligatory subunit of Laminin1. These data indicate that a role for cntn2 in FBM neuron migration can be discerned in a sensitized vang/2+/- background but not in a sensitized lamc1+/- background. In addition to vang/2 and lamc1, we tested for genetic interactions between cntn2 and other PCP (fzd3a, scrb1) and non-PCP genes (cdh2, gpr125) that have been implicated in FBM neuron migration (Li et al., 2013; Rebman et al., 2016; Stockinger et al., 2011; Wada et al., 2005; Wada et al., 2006). We detected genetic interactions for cntn2 only with vang/2, and not with any of the other genes tested (data not shown). Indeed, vang/2 is rather unique in exhibiting genetic interactions with several PCP and non-PCP genes (vang/2; fzd3a and vang/2; 
$c d h 2$, for example), while many of the other combinations (fzd3a; scrb1 or fzd3a; $c d h 2$, for example) also do not exhibit genetic interactions (Gurung, S., Hummel, D., Chandrasekhar, A., in preparation). Given the central role of Vangl2 in Wnt/PCP signaling, only the vang/2+/- background may be sensitive enough to detect a subtle role for Cntn2 in FBM neuron migration.

\subsubsection{Role for Cntn2 in nucMLF development}

The nucMLF neurons extend dendrites toward retino-recipient areas and project axons to the spinal cord (Gahtan et al., 2005; Kimmel et al., 1982), and have been implicated in modulating locomotor speed, optomotor response, escape swimming and prey capture (Sankrithi and O'Malley, 2010; Severi et al., 2014). Morpholino-mediated knockdown experiments suggested roles for cntn2 in the initial oriented growth of nucMLF axons, and their convergence into a tight fascicle (Wolman et al., 2008). We show here that MZcntn2 mutants do not recapitulate this nucMLF morphant phenotype. However, MLF axons in cntn2 mutants do exhibit defasciculation in the midbrain and anterior hindbrain (rhombomeres 1-2). These data suggest that Cntn2 facilitates adhesions between MLF axons, which is consistent with its ability to interact homophilically, as well as heterophilically with other cell adhesion molecules (Brummendorf and Rathjen, 1996).

FBM neurons migrate in close proximity to MLF axons along the entire pathway from r4 to r7 (Bingham et al., 2005; Wada et al., 2006; Wanner and Prince, 2013). The migration of FBM neurons from r5 to r6 depends upon interactions between FBM 
neurons and MLF axons mediated by the cell adhesion molecule Cadherin2 (Cdh2) (Wanner and Prince, 2013). Since MLF axons are frequently defasciculated in cntn2 mutants, it is possible that the interactions between FBM neurons and MLF axons are also partially disrupted in cntn2 mutants. It will be interesting to investigate potential roles for cntn2 and $c d h 2$ in regulating FBM neuron migration indirectly through MLF axon-FBM neuron interactions.

\subsubsection{Role for Cntn2 in sensorimotor circuits}

In the mouse Cntn2 mutant, retinal ganglion cell (RGC) sensory axons, which normally express Cntn2, lose their preference to extend on Cntn2-coated substrates, suggesting that RGC axon fasciculation requires homophilic Cntn2 interactions (Chatzopoulou et al., 2008). In zebrafish, Cntn2 is expressed on RGC neurons and axons (Fig. 3.11E-F). Although no obvious defects in RGC fasciculation or pathfinding were evident in MZcntn2 mutants (Fig. 3.12), the RGC axon fascicle was thinner in some cases, suggesting possible effects on outgrowth. We tested for a potential role in the visual system by measuring swimming activity in light and dark phases. While swimming duration and distance were significantly decreased in $M Z$ mutants compared to controls in both light and dark conditions, both measures of swimming were similarly affected in control and MZ mutants when switching from light to dark phase, indicating that the regulation of motor activity by the visual system was not affected in mutants. It is more likely that the swimming deficits are generated by deficits in circuits downstream of the visual system for two reasons. First, given the nucMLF fasciculation defects in 
MZcntn2 mutants, their swimming deficits are consistent with the role of the nucMLF in modulating locomotion in larval zebrafish (Severi et al., 2014). Second, some of the swimming deficits in $M Z$ mutants may be attributed to a role for cntn2 in the outgrowth or function of sensory neurons in the spinal motor circuits. While we did not find any growth defects in Rohon-Beard (RB) central axons in MZcntn2 mutants (Fig. 3.8), as seen previously in cntn2 morphants (Liu and Halloran, 2005), other aspects of RB morphology or differentiation could potentially be affected. Therefore, our finding that cntn2 mutants exhibit weaker escape responses to trunk touch compared to wildtype controls supports a role for cntn2 in the function of the RB sensorimotor circuit. Since there are no defects in motor and RB axon outgrowth in cntn2 mutants, defects in the connectivity and/or function of sensorimotor components seem more likely. Indeed, some of the motor deficits seen in the mouse Cntn2 mutant (Savvaki et al., 2008) may result from improper development or function of dorsal root ganglion sensory axons (Kunz et al., 1998; Law et al., 2008).

In conclusion, our characterization of the cntn2 CRISPR mutant has revealed definitive roles for the Cntn2 cell adhesion molecule in the zebrafish nervous system. It will be of interest to identify other membrane proteins that function in concert with Cntn2 to regulate FBM neuron migration and MLF axon fasciculation in zebrafish.

\subsection{ACKNOWLEDGEMENTS}

We thank members of the Chandrasekhar lab for discussion and fish care. We thank Dr. Clarissa Henry (University of Maine, Orono) for providing the sleepy (lamc1) 
mutant fish, and Dr. Claudia Stuermer (University of Konstanz) for providing the Cntn2 antibody. We would also like to thank Dr. Jimann Shin (Lila Solnica-Krezel lab, Washington University) for reagents, help and advice on CRISPR protocols, and Dr. Martha Bagnall (Washington University) for help and advice on touch-evoked escape response experiments. This work was supported by NIH grants R01NS086934 (MH) and R01NS040449 (AC), and bridge funds from the University of Missouri Research Board and the Bond Life Sciences Center (AC). 


\subsection{FIGURES AND LEGENDS}

Figure 3.1: Cntn2 protein expression during embryonic development
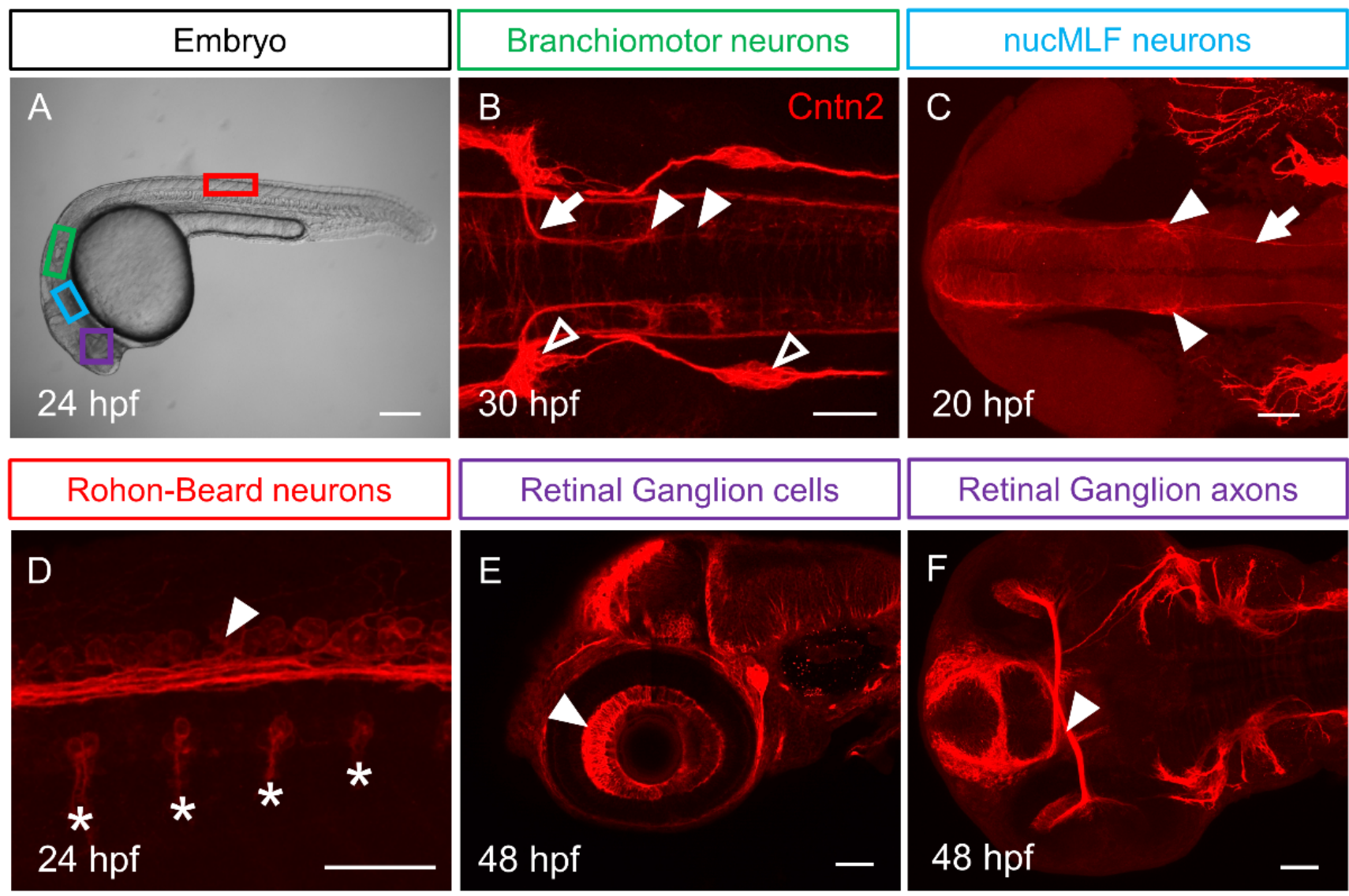

Retinal Ganglion axons

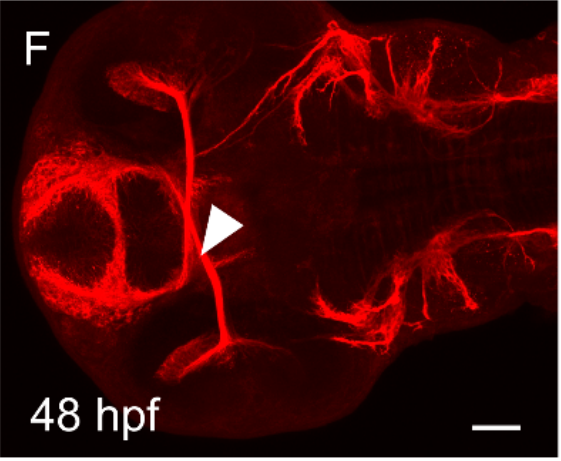


Figure 3.1: Cntn2 protein expression during embryonic development

(A) Lateral view of a $24 \mathrm{hpf}$ embryo with boxes indicating the location of different neuronal cell types in B-F. Panels B-F show confocal projections of embryos labeled with anti-Cntn2 antibody (red). Panel B shows a dorsal view, and $\mathrm{C}$ and $\mathrm{F}$ show ventral views. Panels $D$ and $E$ show lateral views. All panels show anterior to the left. (B) A 30 hpf embryo showing Cntn2 expression in FBM neuron cell bodies in $\mathrm{r} 6$ and $\mathrm{r} 7$ (arrowheads), and their axons (arrow). Open arrowheads mark sensory ganglia. (C) A 20 hpf embryo showing Cntn2 expression in nucMLF cell bodies (arrowheads), and their axons (arrow). (D) A 24 hpf embryo showing Cntn2 expression in central axons (arrowhead) of Rohon-Beard (RB) neurons. Asterisks indicate axons of primary motor neurons exiting the spinal cord. (E, F) A 48 hpf embryo showing Cntn2 expression in nasal RGC (arrowhead, E) as well as RGC axons (arrowhead, F). Scale bars: A, 200 $\mu \mathrm{m}$, and $\mathrm{B}-\mathrm{F}, 50 \mu \mathrm{m}$. 
Figure 3.2: Generation and validation of CRISPR-generated cntn2 mutant

A

cntn2 (86.708kb)

I

sgRNA

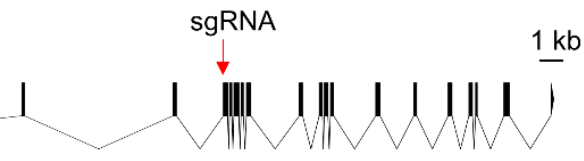

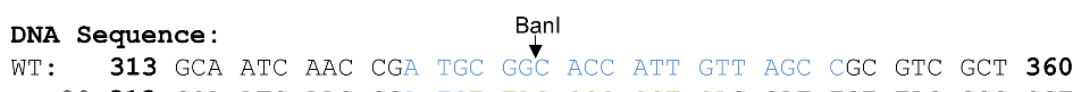

zou20:313 GCA ATC AAC CGA TGT TAG CCG CGT CAC CAT TGT TAG CCG CGT CGC T 367 +7bp(-5,+12)

zou22:313 GCA ATC A----------AC CAT TGT TAG CCG CGT CGC T $349-11 \mathrm{bp}$

Blue: Target sequence -----: Deletion Green: Insertion

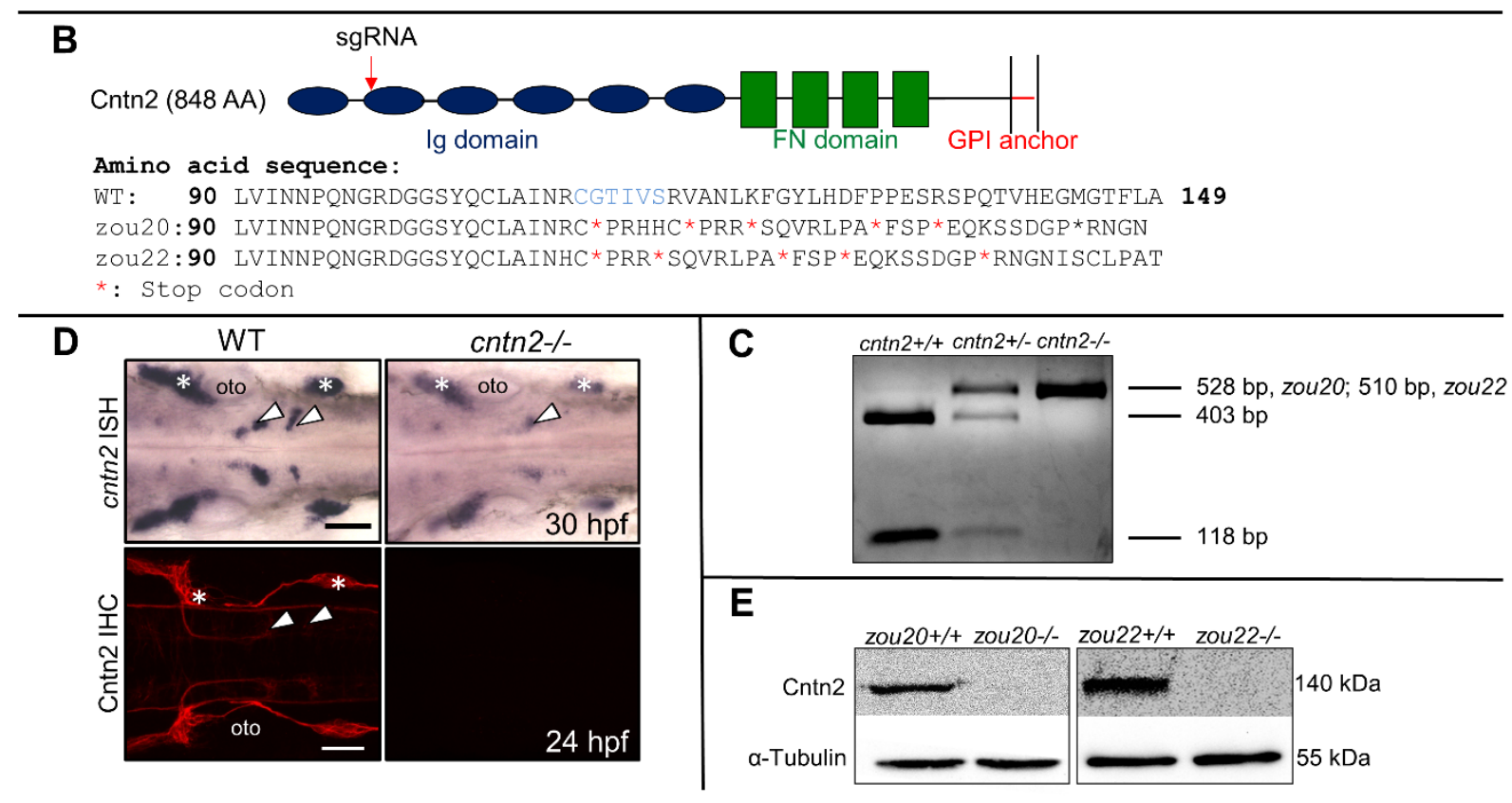


Figure 3.2: Generation and validation of CRISPR-generated cntn2 mutant (A) Genomic structure of cntn2 containing 19 exons, with the CRISPR target site (sgRNA) in the $4^{\text {th }}$ exon. The target site is highlighted in blue while the $7 \mathrm{bp}$ insertion and the $11 \mathrm{bp}$ deletion in cntn $2^{z 0 u 20}$ and $c n t n 2^{\text {zou22 }}$ alleles are highlighted in green and indicated by dash marks, respectively. (B) Domain structure of Cntn2 containing six immunoglobulin (Ig) domains, four fibronectin (FN) domains, and a glycosylphosphatidylinositol (GPI)-anchor linked to the plasma membrane. Predicted amino acid sequences of wildtype (WT) cntn2, and $c n t n 2^{\text {zou20 }}$ and $c n t n^{\text {zou22 }}$ alleles containing multiple stop codons $\left(^{*}\right)$. The highlighted AAs (blue) correspond to the CRISPR target site in the gene. (C) A PCR product (528bp, zou20; 510bp, zou22) spanning the target site digested with Banl differentiate between three genotypes: Wildtype ( 2 cut bands), heterozygote ( 1 uncut and 2 cut bands) and homozygote ( 1 uncut band). (D) Dorsal views of wildtype (WT) and mutant (zou20) hindbrains processed for cntn2 in situ hybridization (ISH) (upper panels) and anti-Cntn2 immunohistochemistry (IHC) (lower panels). Arrowheads indicate migrated FBM neurons and asterisks mark sensory ganglia. In cntn2 (zou20) mutants, cntn2 expression is greatly reduced, and Cntn2 protein is not detectable. Scale bar $=50 \mu \mathrm{m}$.

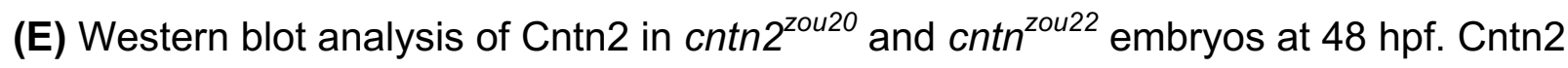
protein is not detectable in cntn2 mutants. Loading control is $\alpha$-tubulin. 
Figure 3.3: Knock down of Cntn2 expression with antisense MO
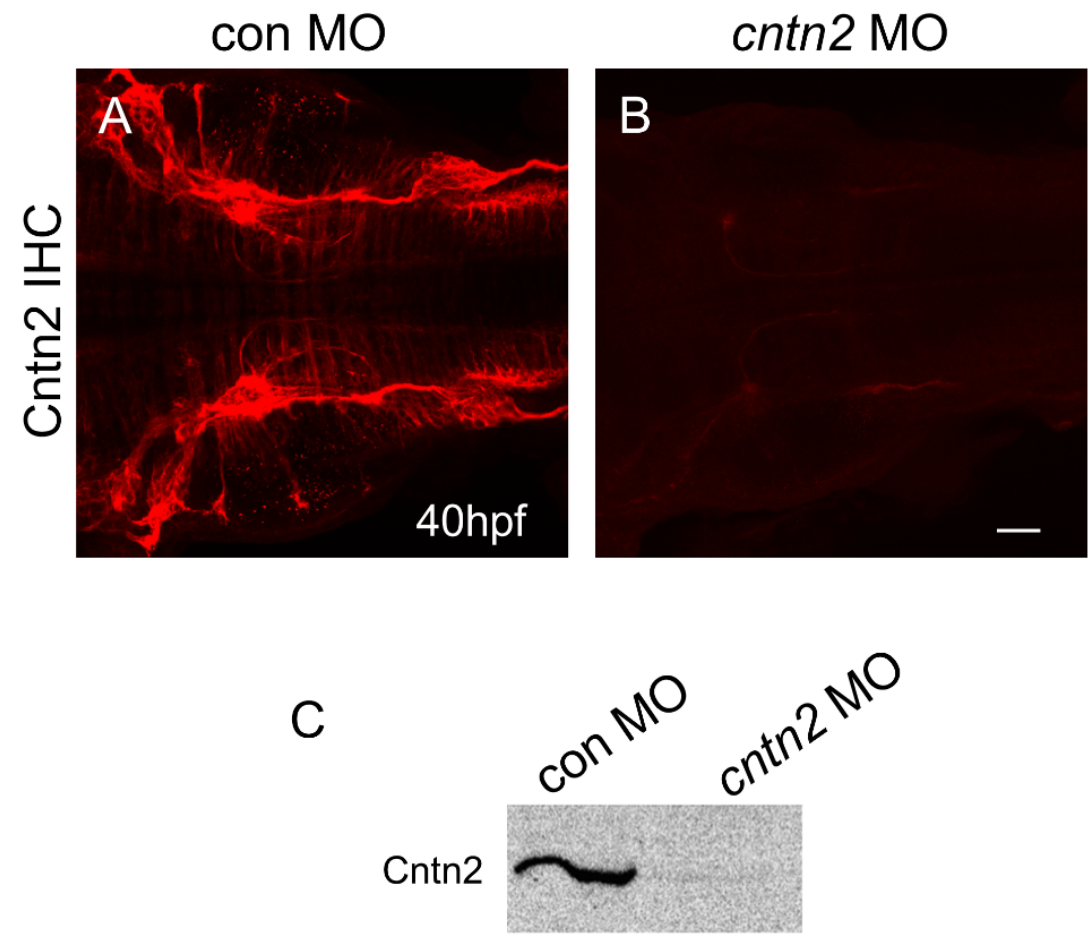

Tubulin 
Figure 3.3: Knock down of Cntn2 expression with antisense MO

Top panels show dorsal view of hindbrain, with anterior to the left, in embryos labeled with the anti-Cntn2 antibody. (A, B) Cntn2 is highly expressed in a control MO-injected embryo (A). Cntn2 expression is almost completely eliminated in a cntn2 MO-injected embryo (B). (C) Western blot analysis shows severe reduction of Cntn2 protein in cntn2 MO-injected embryos at $48 \mathrm{hpf}$. Scale bar in B, $50 \mu \mathrm{m}$ for A and B. 
Figure 3.4: FBM neuron migration is affected in cntn2 morphants but not in cntn2 mutants
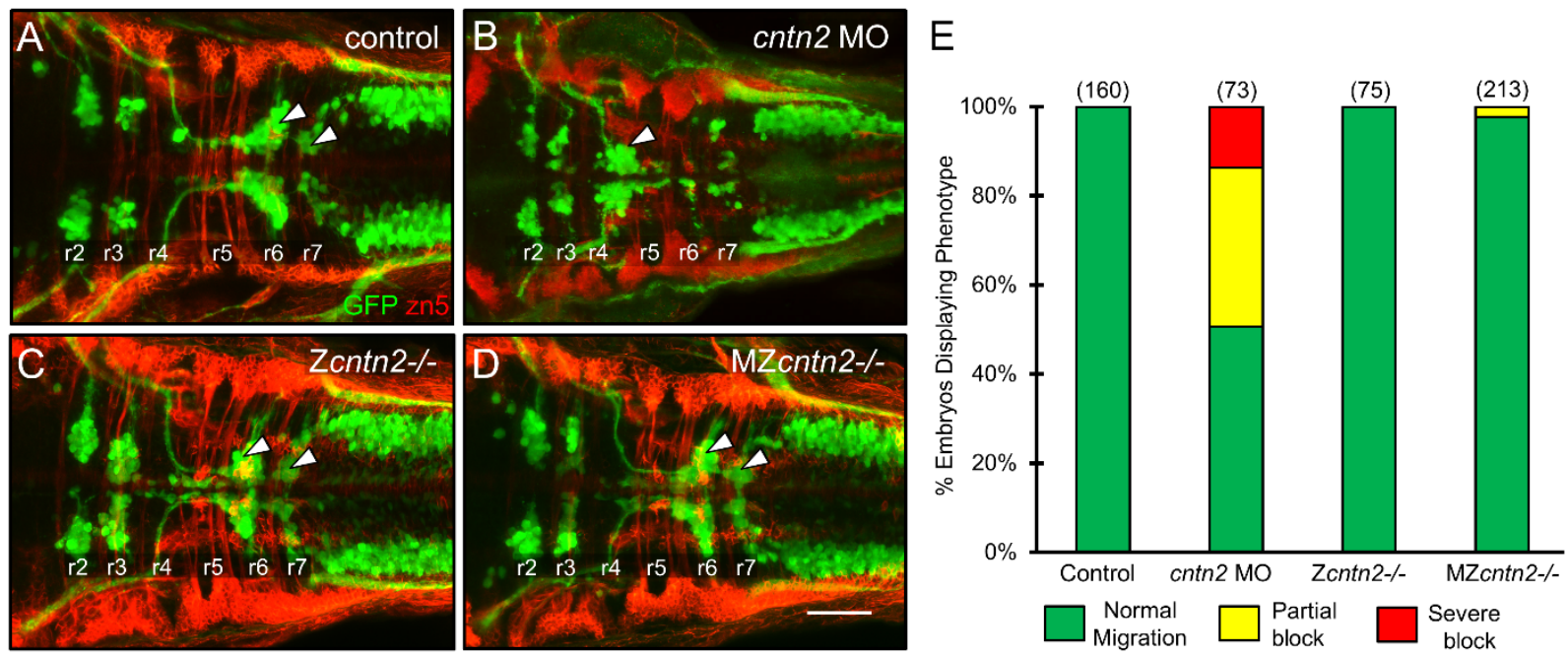
Figure 3.4: FBM neuron migration is affected in cntn2 morphants but not in cntn2 mutants

Panels A-D show dorsal views of the hindbrain with anterior to the left. $T g(i s / 1: g f p)$ embryos were fixed at $48 \mathrm{hpf}$, and processed for immunohistochemistry with zn5 antibody (red) to label hindbrain commissural neurons and axons at rhombomere boundaries, and anti-GFP antibody (green) to label FBM neurons (arrowheads). (A) FBM neurons (arrowheads) migrate normally into $r 6$ and $r 7$ in an uninjected embryo. (B) FBM neurons largely fail to migrate out of $\mathrm{r} 4$ in a cntn2 MO-injected embryo. (C, D) FBM neurons migrate normally in zygotic mutant (Zcntn2-/-) (C), and maternal-zygotic mutant (MZcntn2-/-) (D) embryos. Scale bar in D, $50 \mu \mathrm{m}$ for A-D. (E) Quantification of FBM neuron migration defects. Number in parenthesis denotes number of embryos. Data are from 3 to 4 experiments. 
Figure 3.5: cntn2 interacts genetically with vang/2 but not with lamc1
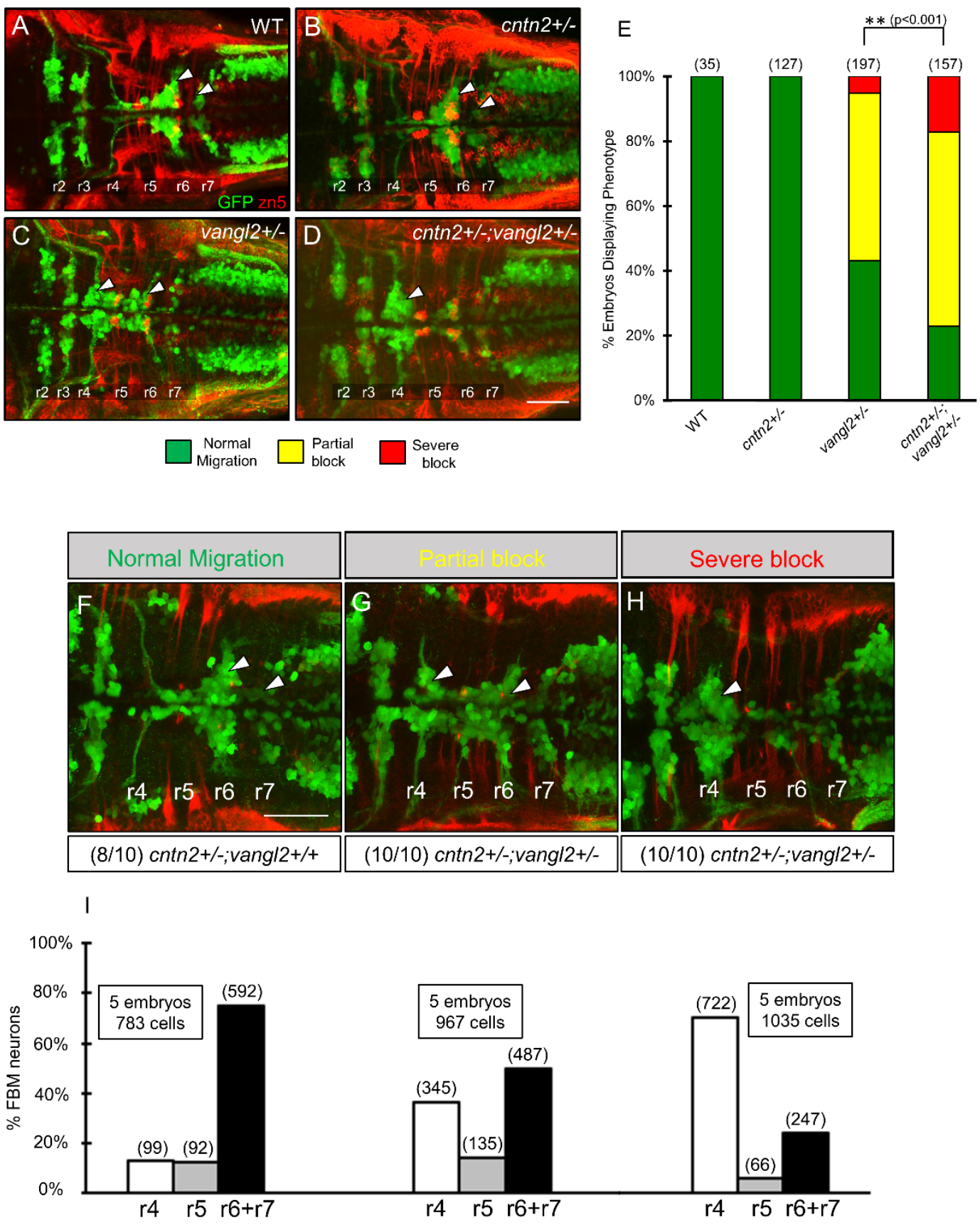


\section{Figure 3.5: cntn2 interacts genetically with vang/2 but not with lamc1}

Panels A-D and F-H show dorsal views of the hindbrain with anterior to the left.

$T g$ (is/1:gfp) embryos were fixed at $48 \mathrm{hpf}$, and processed for immunohistochemistry with zn5 antibody (red) to label hindbrain commissural neurons and axons at rhombomere boundaries, and anti-GFP antibody (green) to label FBM neurons (arrowheads). (A) FBM neurons migrate normally in a control embryo. (B) FBM neurons migrate normally in a cntn2 heterozygous (cntn2+/-) embryo. (C) FBM neurons migrate poorly in a vang/2 heterozygous (vang/2+/-) embryo, with neurons located along the entire migratory pathway from r4 to r6. (D) FBM neurons fail to migrate out of r4 in a cntn2; vang/2 double heterozygote (cntn2+/-; vang/2+/-). Scale bar in D, $50 \mu \mathrm{m}$ for A-D. (E) Quantification of genetic interaction data. Number in parenthesis denotes number of embryos. ${ }^{* *}$ Chi-square test at $p<0.001$; NS: not significant. Data are from 2 to 4 experiments. (F-H) Offsprings of vang/2+/- heterozygous and cntn2-/- homozygous mutants exhibit normal, partial block, and severe block phenotypes for FBM neuron migration. Embryos exhibiting partial block (10/10) and severe block (10/10) were all identified as cntn2; vang/2 double heterozygote (cntn2+/-; vang/2+/-) and a majority of embryos (8/10) exhibiting normal migration were identified as cntn2+/-; vang/2+/+ by genotyping. (I) Quantification of non-migrated FBM neurons in r4, partially migrated FBM neurons in $\mathrm{r} 5$ and fully migrated FBM neurons in $\mathrm{r} 6$, and $\mathrm{r} 7$. Number in parenthesis denotes number of cells. Scale bar in F, $50 \mu \mathrm{m}$ for F-H. 
Figure 3.6: cntn2 mutants show MLF defasciculation but lacks nucMLF defects seen in morphants

nucMLF axon convergence
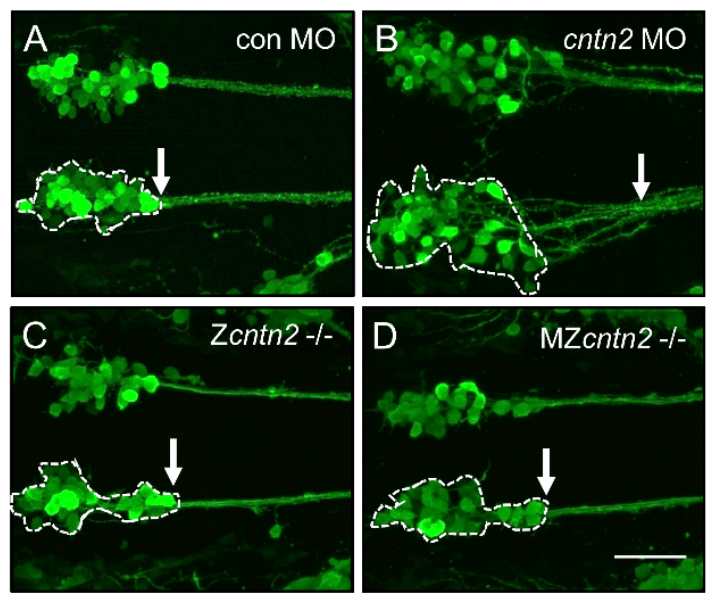

E

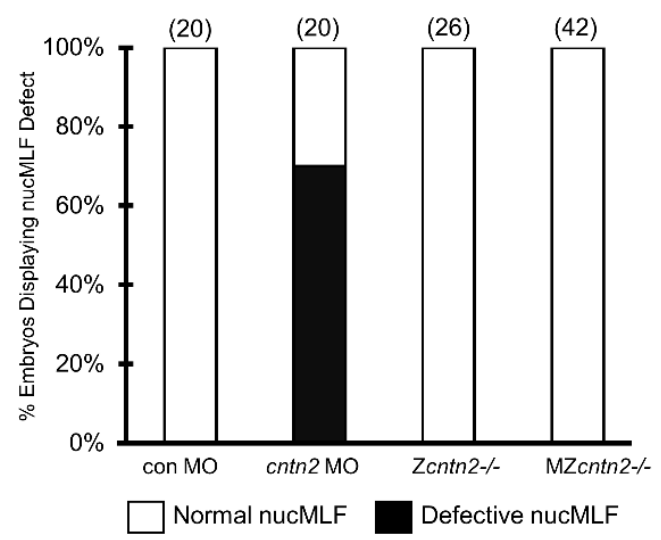

MLF fasciculation

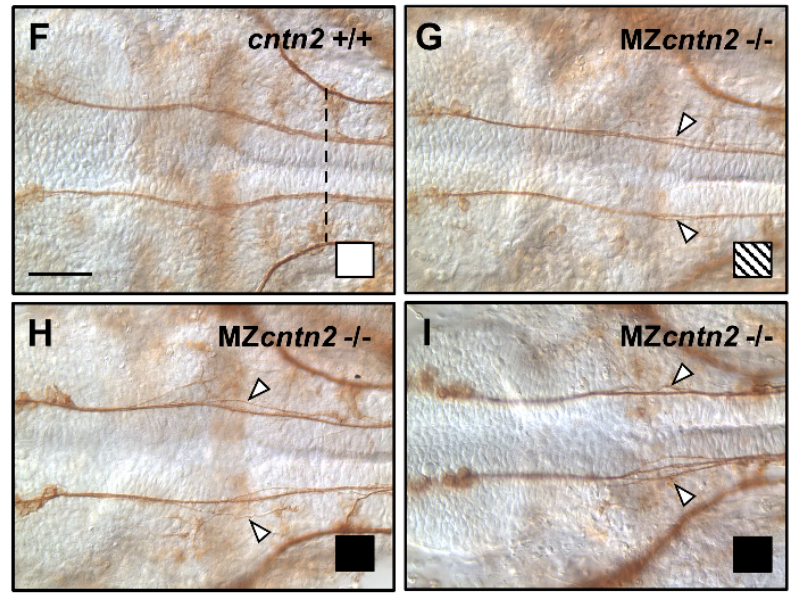

$J$

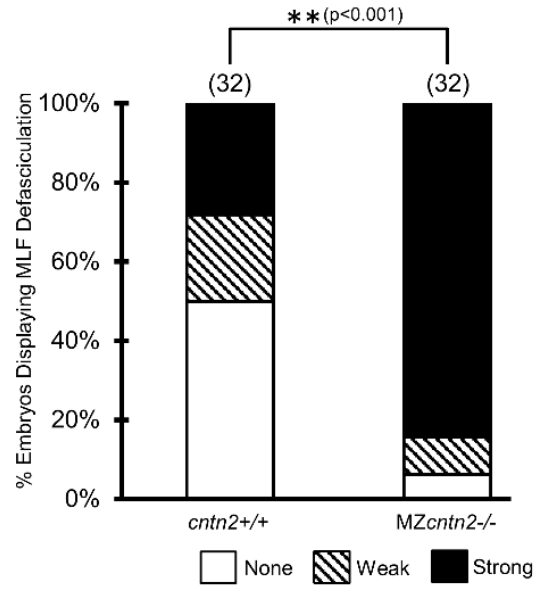


Figure 3.6: cntn2 mutants show MLF defasciculation but lacks nucMLF defects seen in morphants

Panels A-D show ventral views of the midbrain with anterior to the left. (A-D) Confocal projections of $T g$ (pitx2c:gfp) embryos labeled with anti-GFP antibody at 24 hpf. Panels F-I show ventral views of the midbrain and anterior hindbrain region with anterior to the left. (A) In a control MO-injected embryo, the nucMLF is found as bilateral groups of tightly clustered cells (delineated by dashed outline). Their axons form tight fascicles (arrow) immediately posterior to the neuron clusters. (B) In a cntn2 MO-injected embryo, the nucMLF neurons are loosely packed, and their axons are defasciculated. (C, D) The nucMLF neurons and axons converge normally in zygotic (Zcntn2-/-) and maternal-zygotic (MZcntn2-/-) mutants. (E) Quantification of nucMLF defects. Number in parenthesis denotes number of embryos. Data are from 2 to 4 experiments. (F-I) Zn-12 antibody labeling of the MLF axons in 24 hpf embryos. MLF axons in a cntn2+/+ form a tight fascicle (F); however, MLF axons are defasciculated (arrowheads) in MZcntn2-/embryos (G-I). Black dotted line in F shows the cut-off point (for scoring) where the trigeminal sensory axons enter the hindbrain in r2. (J) Quantification of MLF defasciculation defects. Number in parenthesis denotes number of embryos. Data are from 2 experiments. Scale bar in D, $50 \mu \mathrm{m}$ for A-D; Scale bar in F, $50 \mu \mathrm{m}$ for F-I. 
Figure 3.7: Some neuronal defects in cntn2 morphants are likely to be off-target effects

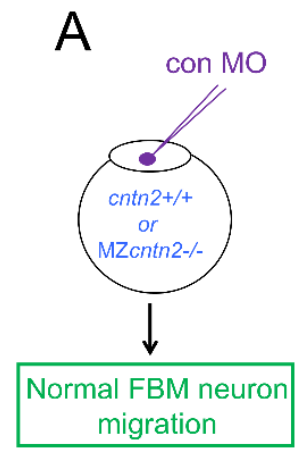

Normal nucMLF

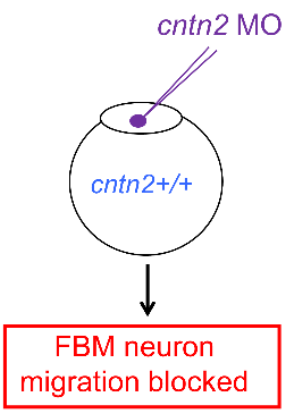

nucMLF defect
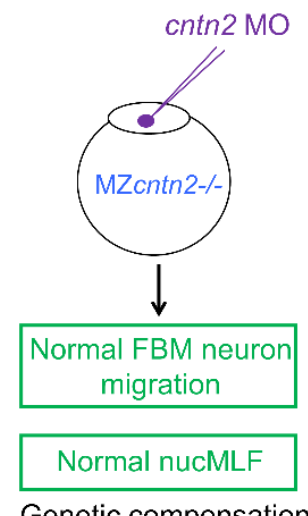

Genetic compensation of cntn2 null
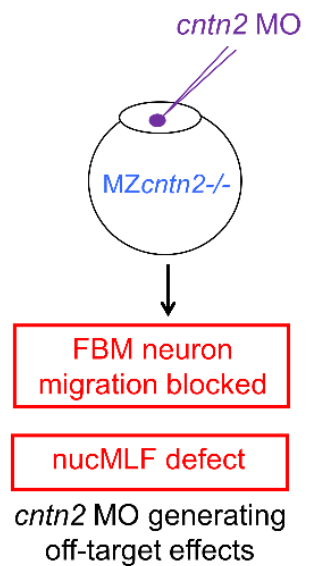

FBM neuron migration
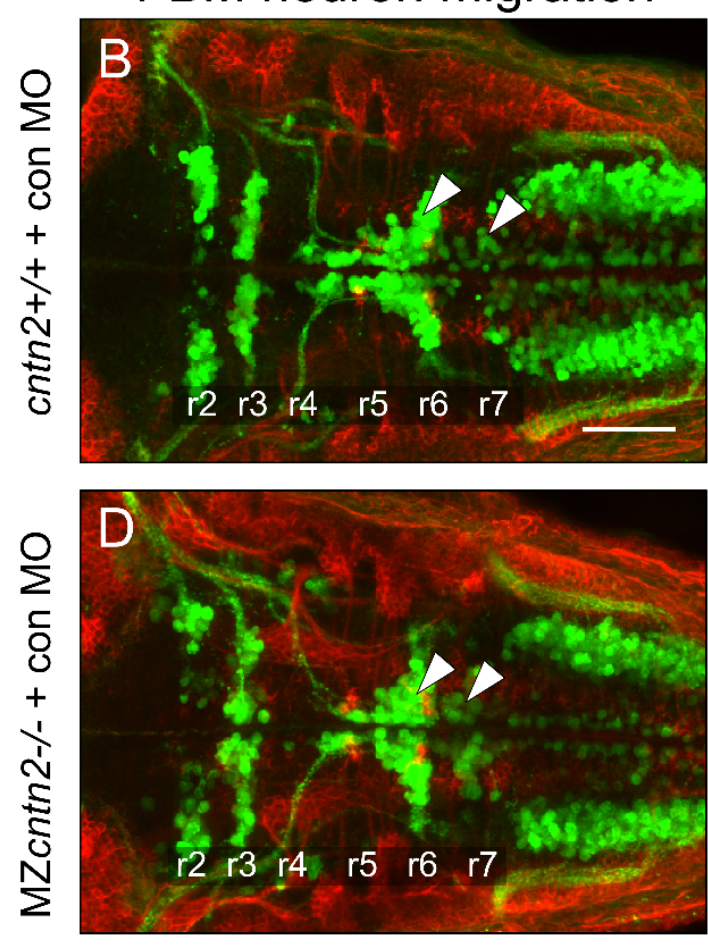
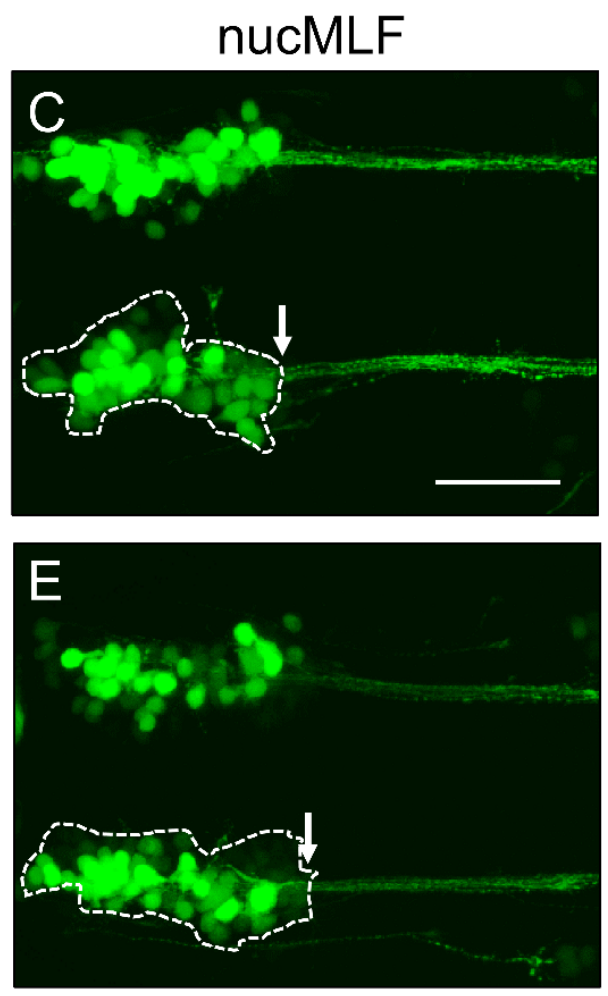
Figure 3.7: Some neuronal defects in cntn2 morphants are likely to be off-target effects (continued)
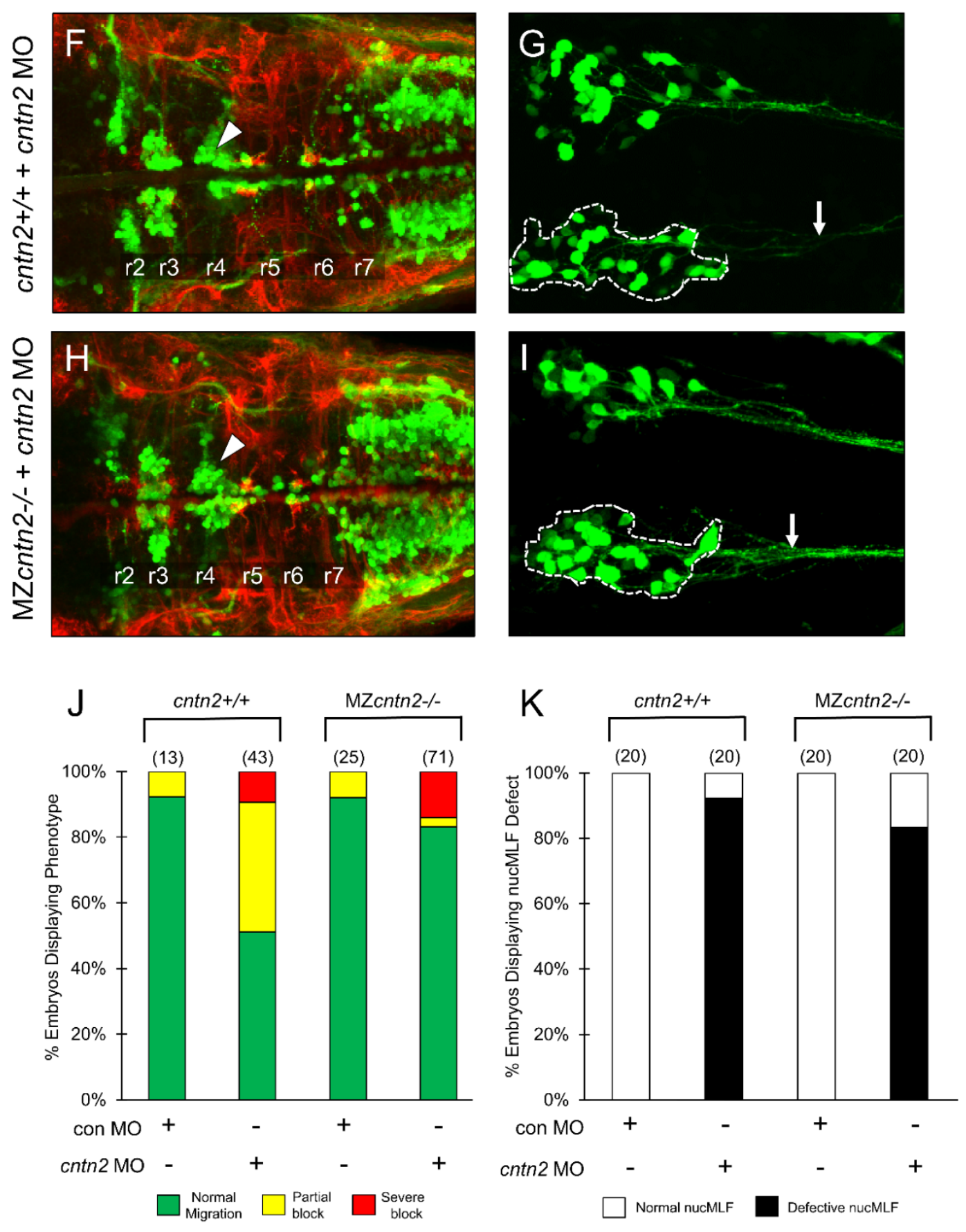
Figure 3.7: Some neuronal defects in cntn2 morphants are likely to be off-target effects

(A) Experiments to distinguish between genetic compensation in MZcntn2-/- mutants, and off-target effects of the cntn2 MO. Normal development of FBM and nucMLF neuron in MZcntn2-/- mutants injected with cntn2 MO would suggest compensation. However, defective development of both cell types in these embryos would suggest offtarget effects. $(B, D, F, H)$ Dorsal views of the hindbrain with anterior to the left. $T g$ (is/1:gfp) embryos were fixed at $48 \mathrm{hpf}$, and processed for immunohistochemistry with zn5 antibody (red) to label hindbrain commissural neurons and axons at rhombomere boundaries, and anti-GFP antibody (green) to label FBM neurons (arrowheads). (B, D) FBM neurons migrate normally in control MO-injected cntn2+/+ (B) and in MZcntn2-/(D) embryos. $(\mathbf{F}, \mathbf{H})$ Migration of FBM neurons is greatly reduced in cntn2 MO-injected cntn2+/+ $(F)$ and MZcntn2-/- $(H)$ embryos. $(C, E, G, I)$ Ventral views of the midbrain, with anterior to the left, of $T g$ (pitx2c:gfp) embryos labeled with anti-GFP antibody. (C, E) Normal nucMLF development in control MO-injected cntn2+/+ (C) and MZcntn2-/- (E) embryos. (G, I) Defective nucMLF development in cntn2 MO-injected cntn2+/+ (G) and MZcntn2-/- (I) embryos. (J) Quantification of data presented in B, D, F and H. Number in parenthesis denotes number of embryos. (K) Quantification of data presented in C, E, G and I. Number in parenthesis denotes number of embryos. Scale bar in B, $50 \mu \mathrm{m}$ for B, $D, F$, and $H$; Scale bar in $F, 50 \mu m$ for $C, E, G$, and I. 
Figure 3.8: Outgrowth of Rohon-Beard central axons is not affected in MZcntn2 mutants

A

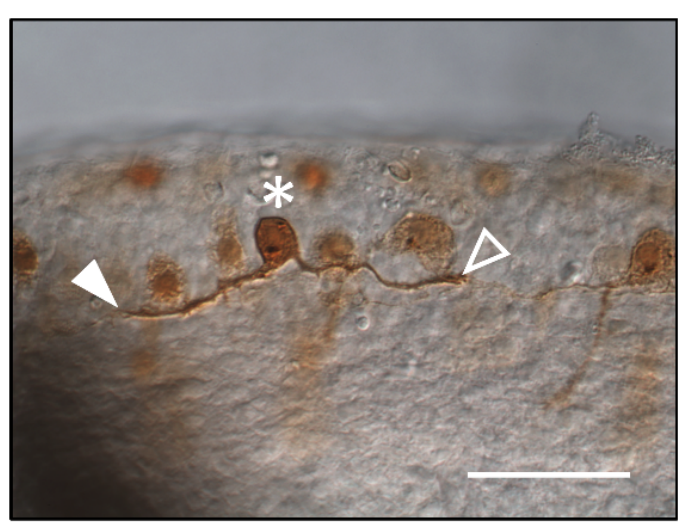

C

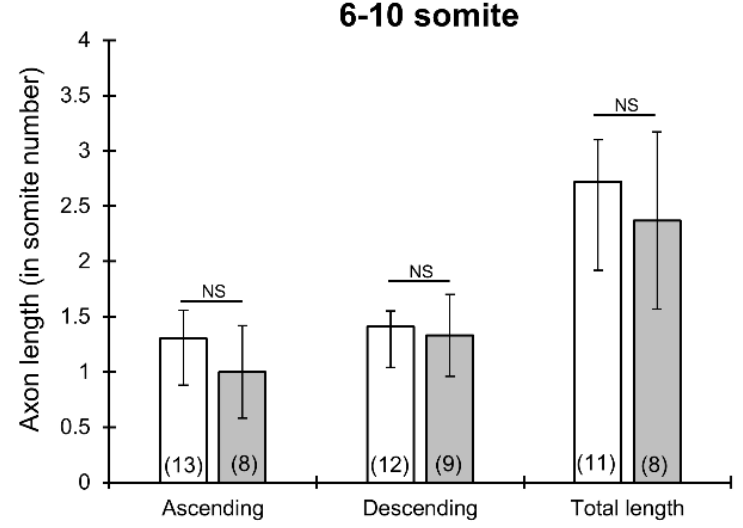

B

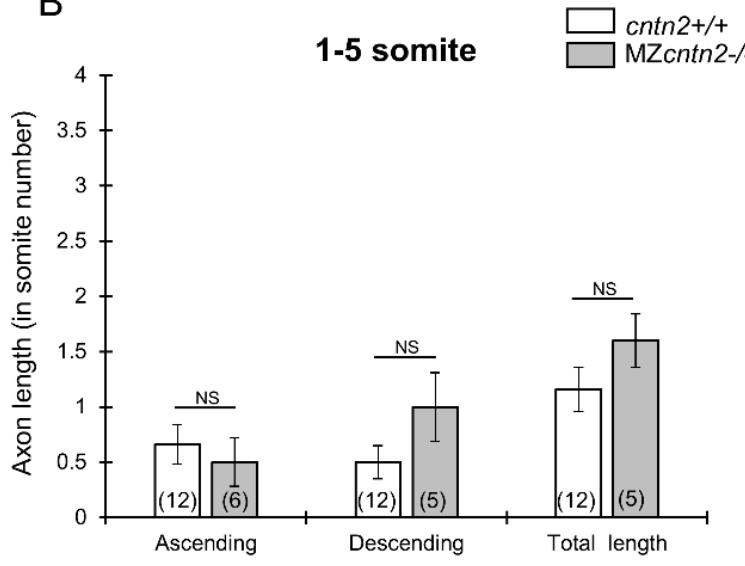

D

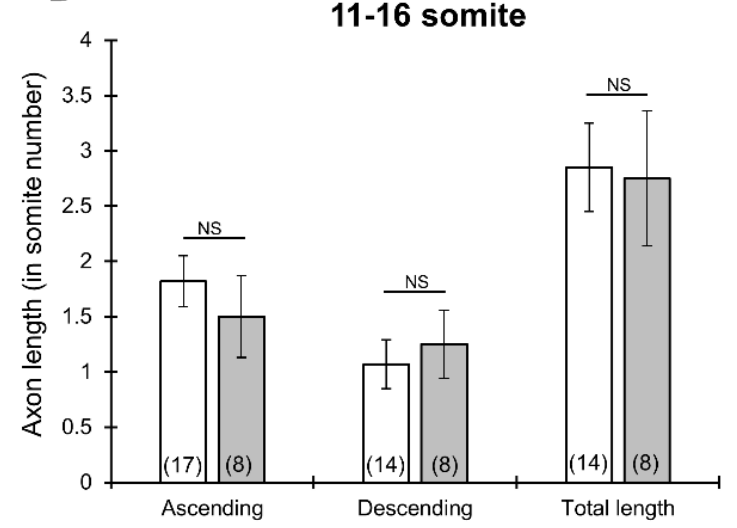


Figure 3.8: Outgrowth of Rohon-Beard central axons is not affected in MZcntn2 mutants

(A) Embryos injected with ngn1:GFP-caax DNA and Tol2 transposase RNA were fixed at $18 \mathrm{hpf}$, and processed for immunohistochemistry with anti-GFP antibody to label Rohon-Beard (RB) neurons . Asterisk indicates cell body, and the filled and open arrowheads indicate ascending and descending central axons, respectively, of a labeled RB neuron. Scale bar, $50 \mu \mathrm{m}$. (B-D) Quantification of the lengths of ascending and descending central axons, and total length (sum of ascending and descending lengths) of central axons of RB neurons located in the anterior (somites 1-5), intermediate (somite 6-10) and posterior (somites 11-16) regions of the spinal cord. There were no significant differences in the length of RB central axons between wildtype and MZcntn2 mutant embryos. Number of RB neurons scored is shown in parenthesis. Unpaired ttest; NS: not significant. Error bars show Mean \pm SEM. 
Figure 3.9: cntn2 mutants exhibit defective touch responses

A

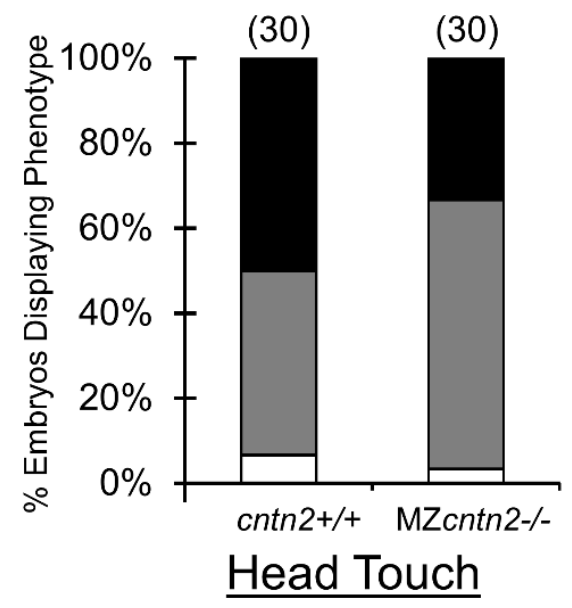

B

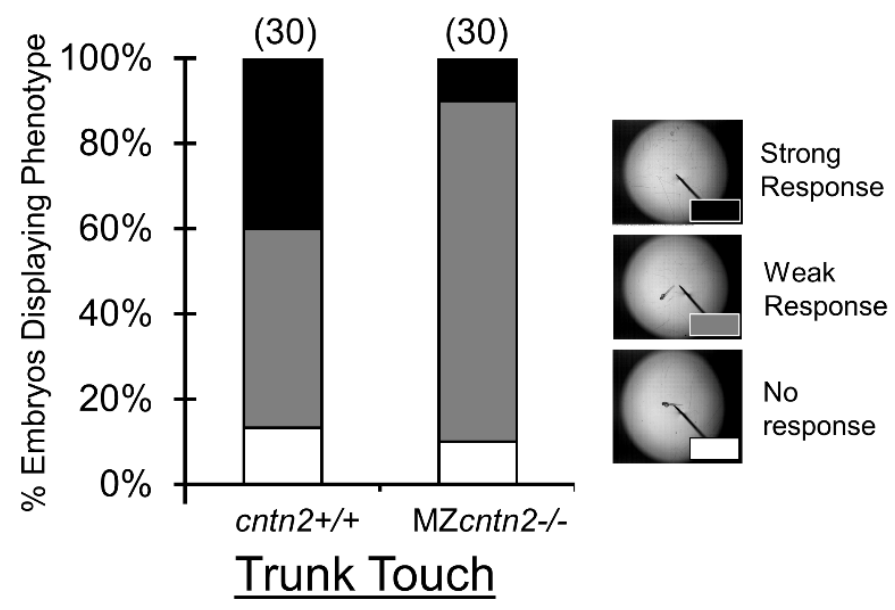


Figure 3.9: cntn2 mutants exhibit defective touch responses

(A, B) Distribution of touch-evoked escape responses of $2 \mathrm{dpf}$ cntn2+/+ and MZcntn2-/embryos following a head touch $(A)$ or a trunk touch $(B)$. The larval responses were binned into three categories: No response (no movement after touch), Weak Response (muted movement with larva remaining in the field of view), and Strong Response (rapid and vigorous movement with larva swimming out of the field of view). MZcntn2-/mutants responded similarly to control cntn2+/+ larvae when touched on the head. However, they exhibited much weaker escape responses compared to cntn2+/+ larvae when touched in the trunk. Data pooled from 2 experiments (number of embryos in parenthesis). ${ }^{*}$ Chi-square test at $p<0.05$; NS: not significant. 
Figure 3.10: Spinal motor axons develop normally in cntn2 mutants
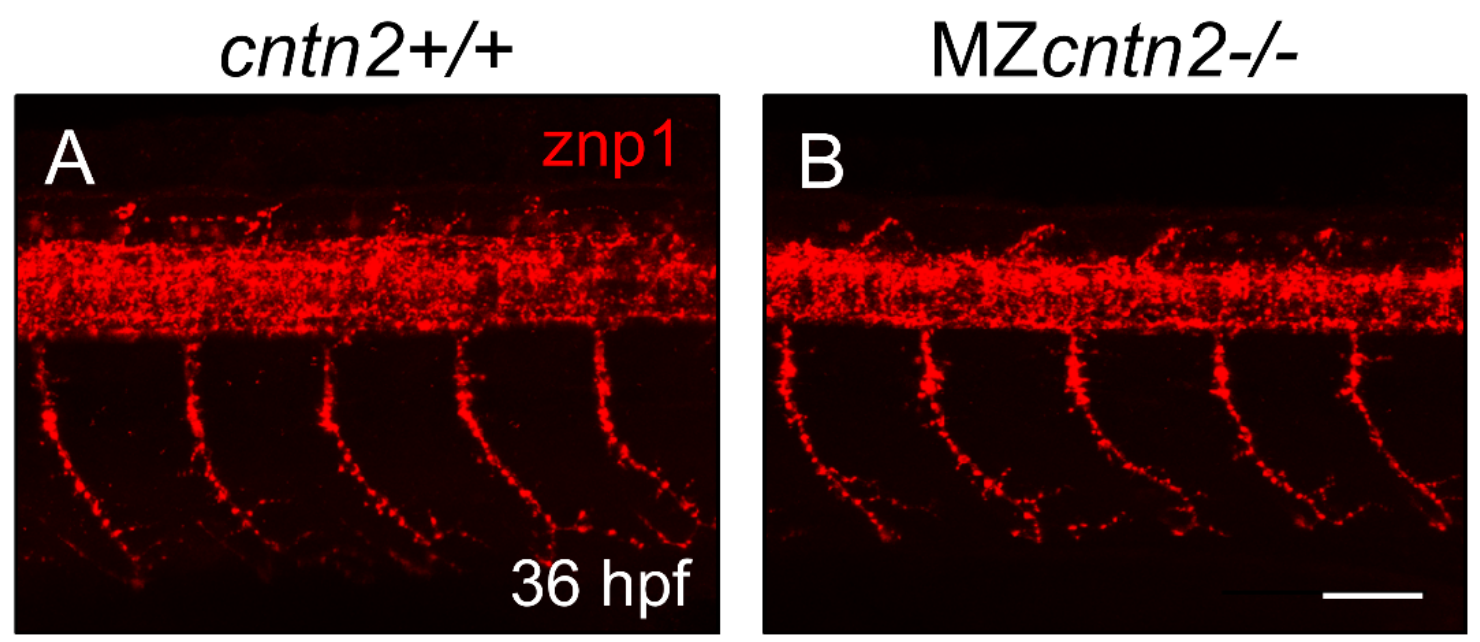
Figure 3.10: Spinal motor axons develop normally in cntn2 mutants

Lateral views of the trunk with anterior to the left, in $36 \mathrm{hpf}$ embryos stained with znp1 antibody. (A) In a wildtype embryo, the motor axon fascicles have extended into the ventral trunk musculature. (B) In a MZcntn2 mutant, the pattern of motor axon outgrowth is not affected. Scale bar in B, $50 \mu \mathrm{m}$ for A and B. 
Figure 3.11: cntn2 mutants exhibit swimming deficits

A

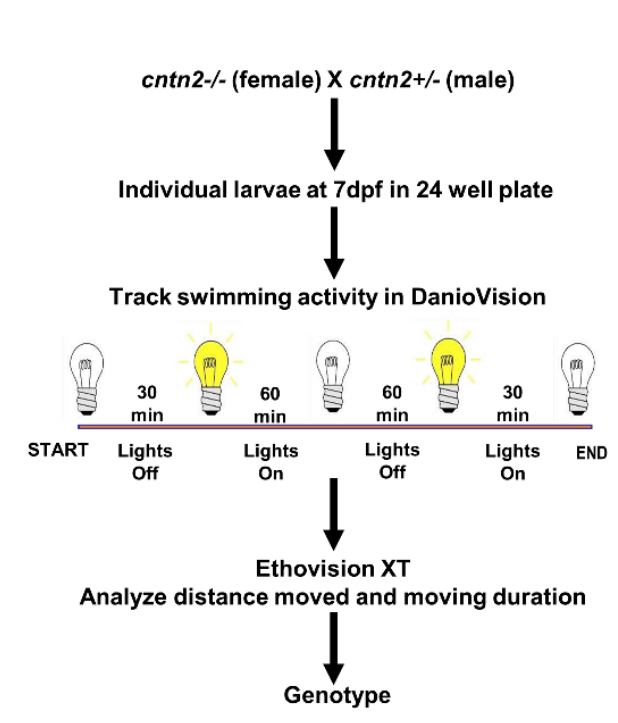

B

\section{Lights off}
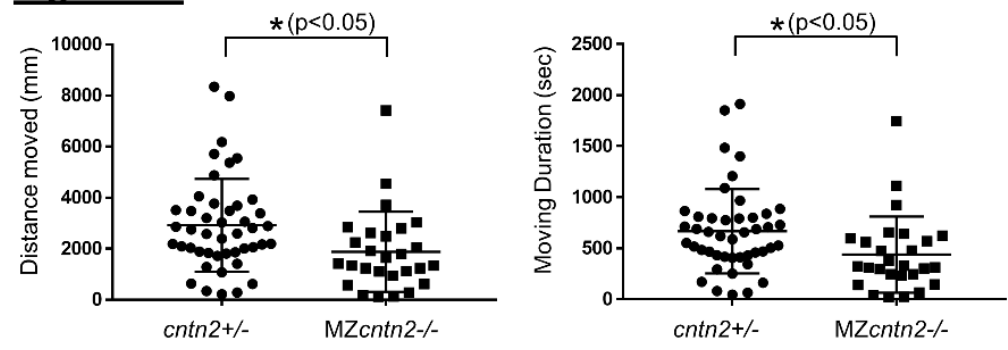

C

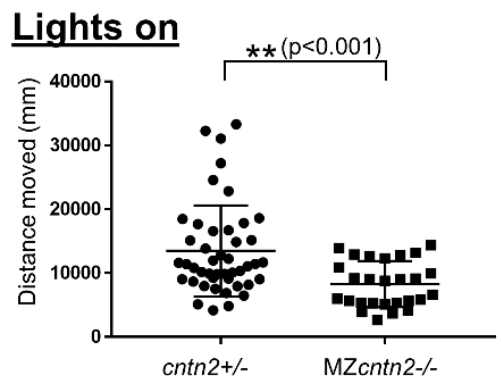




\section{Figure 3.11: cntn2 mutants exhibit swimming deficits}

(A) Swimming assay and analysis. (B, C) Distance moved and moving duration are compared between cntn2+/- heterozygote and MZcntn2-/- mutant larvae during Lights off (B) and Lights on (C) phases. There were significant differences between cntn2+/$(n=43)$ and MZcntn2-/- $(n=27)$. MZcntn2-/- larvae moved less than cntn2+/- siblings during both lights off (unpaired t-test, ${ }^{*} p<0.05$ ) and lights on (unpaired t-test with Welch's correction, $\left.{ }^{* *} \mathrm{p}<0.001\right)$ phases $(\mathrm{B}, \mathrm{C})$. MZcntn2-/- larvae also moved for shorter duration compared to $c n t n 2+/-$ siblings during both lights off (unpaired t-test, ${ }^{*} p<0.05$ ) and lights on (unpaired t-test, ${ }^{* *} p<0.001$ ) phases $(B, C)$. Error bars show Mean $\pm S D$. 
Figure 3.12: Retinal ganglion cell axon fascicles are variably affected in cntn2 mutants
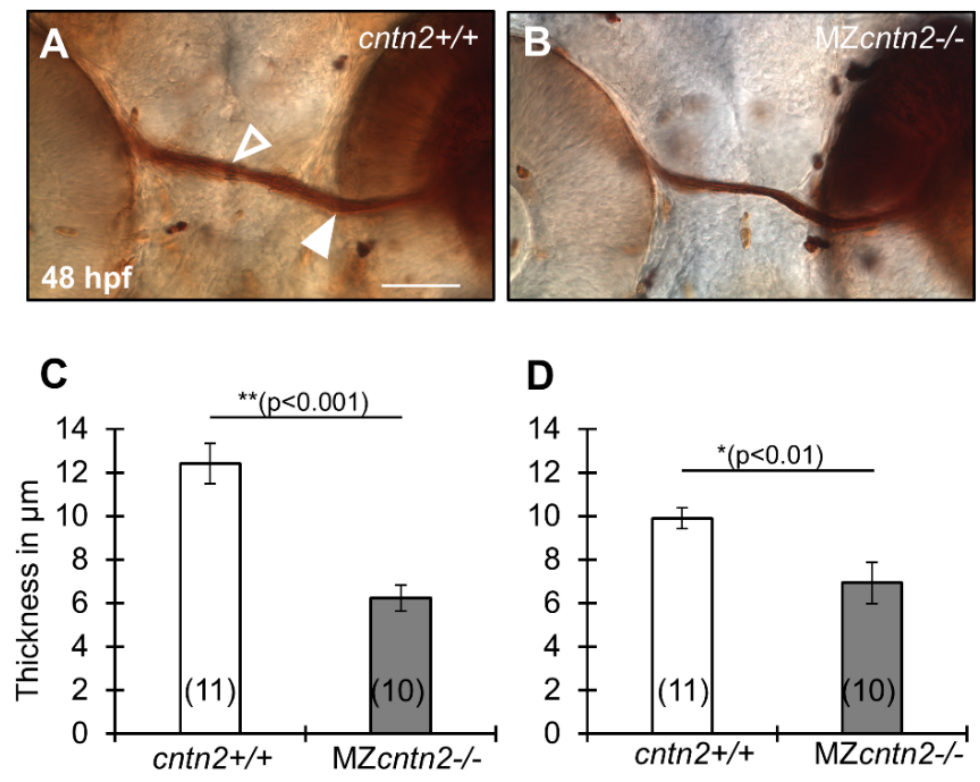

D

Midline

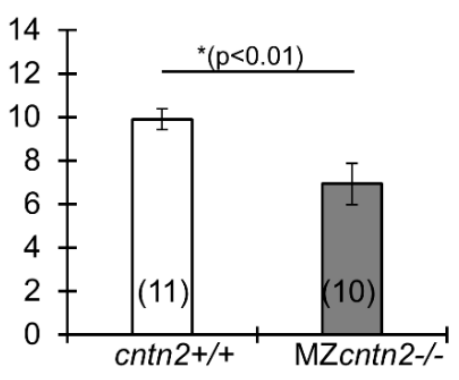

Exit point
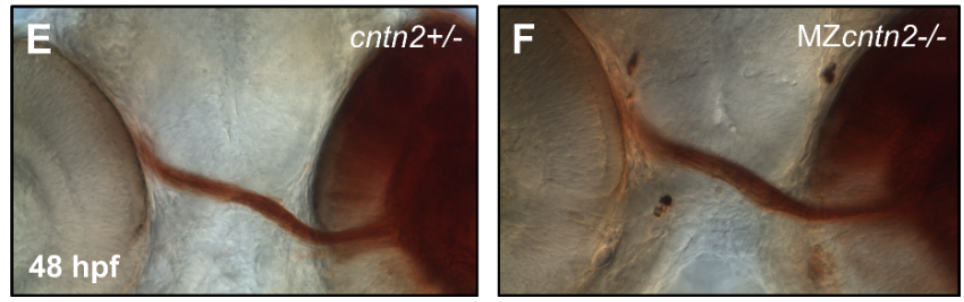

G
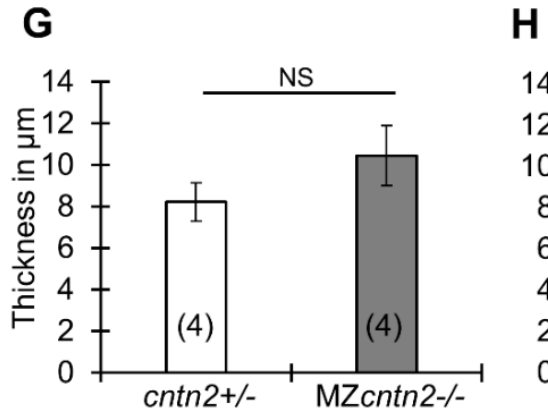

Midline

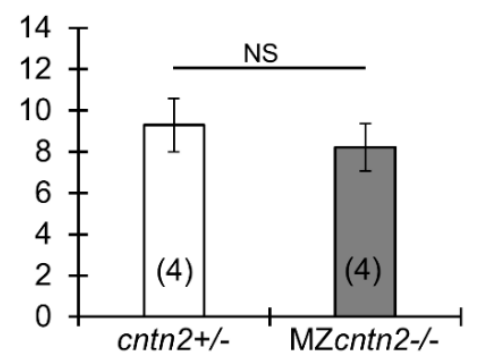

Exit point 
Figure 3.12: Retinal ganglion cell axon fascicles are variably affected in cntn2 mutants

(A, B, E, F) Ventral view of Dil-labeled retinal ganglion cell (RGC) axon fascicles after photoconversion in cntn2+/+ (A), cntn2+/- (E) and MZcntn2-/- (B, F) embryos at $48 \mathrm{hpf}$. The Dil-injected region targeting the nasal RGCs appears as a dark brown area on the right side in each panel. The filled arrowhead marks an RGC axon fascicle at the exit point from the eye, and the open arrowhead marks an RGC axon fascicle at the midline. (C, D, G, H) Summary of RGC axon fascicles thickness in wild-type and MZcntn2-/embryos. The RGC axon fascicles are significantly thinner at the midline (C) and at the exit point from the eye (D) in MZcntn2-/- mutants (B) compared to cntn2+/+ cousins (A). However, these differences were not seen $(G, H)$ when MZcntn2-/- mutants $(F)$ were compared to cntn2+/- siblings (E). Scale bar in A, $50 \mu \mathrm{m}$ for A, B, E, and F. Unpaired ttest; NS: not significant. Error bars show Mean \pm SEM. 


\section{CHAPTER 4: DIFFERENTIAL ROLES FOR SPECIFIC PLANAR CELL POLARITY (PCP) AND NON-PCP GENES DURING}

\section{FACIAL BRANCHIOMOTOR NEURON MIGRATION IN}

\section{ZEBRAFISH}

Suman Gurung ${ }^{1}$, Devynn Hummel ${ }^{1}$, Isabelle Roszko ${ }^{2}$, Diane S. Sepich ${ }^{2}$, Lilianna

Solnica-Krezel ${ }^{2}$, and Anand Chandrasekhar ${ }^{1}$.

1, Division of Biological Sciences and Bond Life Sciences Center, University of Missouri, Columbia, MO 65211, USA.

2, Department of Developmental Biology, Washington University School of Medicine, St Louis, MO 63110, USA.

Corresponding Author:

Dr. Anand Chandrasekhar

Division of Biological Sciences

Room 340D Bond Life Sciences Center

1201 Rollins St

University of Missouri, Columbia, MO 65211-7310

Running Title: Role of PCP and non-PCP genes in facial branchiomotor neuron migration

Keywords: Zebrafish; Neuronal Migration; Facial Branchiomotor Neuron; Wnt/PCP;

Vangl2; Gpr125; Cdh2; Fzd3a; Scrb1; Cntn2; Genetic interaction

\section{Manuscript in preparation}




\subsection{ABSTRACT}

The caudal migration of facial branchiomotor (FBM) neurons in the vertebrate hindbrains are controlled by many genes. Many Wnt/Planar Cell Polarity (PCP) proteins are demonstrated or postulated to act at the cell surface, however, the contributions for the various proteins to neuron migration are unclear. Several but not all genes encoding components of the PCP pathway have been shown to play essential roles in FBM neuron migration in zebrafish. PCP proteins Vangl2, Pk1a, Scrb1, and Fzd3a are necessary while non-PCP proteins Cntn2, Gpr125, and Cdh2 have relatively minor roles for FBM neuron migration. In this study, we have evaluated the relative contributions of the PCP and non-PCP genes by performing several pairwise genetic interaction analyses. Several genetic combinations involving vangl2 with PCP and non-PCP genes generate strong effects on FBM neuron migration. However, most combinations not involving vang/2 generate weak or no effects. Together, Vangl2 appears to play a unique role in FBM neuron migration and suggest that it may play a key role in regulating the function of several PCP and non-PCP genes during FBM neuron migration. 


\subsection{INTRODUCTION}

Neuronal migration is essential for the assembly of neural circuits that control complex behaviors. Many human brain disorders result from incorrect neuronal migration (McManus and Golden, 2005; Vajsar and Schachter, 2006). Therefore, an understanding of the molecular mechanisms regulating neuronal migration can provide insight into the pathological processes of these disorders. Migrating neurons respond to chemotactic cues in the environment and modulate cell-cell interactions with neighboring cells as they navigate from where they are born to where they end up forming functional neural circuits (Marin et al., 2010). During early development of the nervous system, migrating neurons employ two modes of migration: radial (parallel to radial glia) or tangential (orthogonal to radial glia, either occurring along the mediallateral or anterior-posterior axes) (Pearlman et al., 1998; Rakic, 1974). Radially migrating neurons use radial glial fibers as a substrate (Campbell and Gotz, 2002), however, tangentially migrating neurons use interactions with neighboring cells and environmental cues to navigate to their final position. Cell-cell interactions, which are critical for precise neuronal migration, are mediated by a number of membraneassociated molecules (Marin et al., 2010).

Due to their conserved organization as well as the stereotypic migration, the facial branchiomotor (FBM) neurons have become an excellent model to study neuronal migration in the vertebrate embryo (Chandrasekhar, 2004; Song, 2007). Branchiomotor neurons are generated in specific rhombomeres in the hindbrain and innervate muscles 
that arise in the pharyngeal arches (Chandrasekhar et al., 1997; Lumsden and Keynes, 1989; Noden, 1983). In zebrafish, the facial branchiomotor (FBM) neurons undergo a characteristic tangential migration along the rostral-caudal axis from rhombomere $4(\mathrm{r} 4)$ to r6 and r7 where they form the facial motor nucleus (Chandrasekhar et al., 1997; Higashijima et al., 2000). Through forward genetic screens, several genes encoding components of the Wnt/Planar Cell Polarity (PCP) pathway have been shown to play essential roles in FBM neuron migration in zebrafish. The presumptive Wnt receptor Frizzled3a (Wada et al., 2006), the transmembrane protein Vangl2 (Bingham et al., 2002; Jessen et al., 2002), atypical cadherins Celsr 1a, 1b, and 2 (Wada et al., 2006), cytoplasmic adaptors Prickle1a (Carreira-Barbosa et al., 2003), Prickle1b (Mapp et al., 2011), and Scribble1 (Wada et al., 2005) are required for caudal migration of FBM neurons. However, in addition to these PCP genes, several non-PCP genes encoding Laminina1 (Grant and Moens, 2010; Sittaramane et al., 2009), Cadherin2 (Rebman et al., 2016; Stockinger et al., 2011), MAM domain containing glycosylphosphatidylinositol anchor 2A (Mdga2a) (Ingold et al., 2015) , chemokine receptor Cxcr4 (Cubedo et al., 2009), WAVE homology domain protein Nhsl1b (Walsh et al., 2011), RE1-silencing transcription factor (Rest) (Love and Prince, 2015), and elongation factor Spt5 (Cooper et al., 2005) have also been shown to be involved in FBM neuron migration. Interestingly, vang/2 interacts genetically with non-PCP genes like hdac1, which encodes a histone deacetylase (Nambiar et al., 2007), cntn2, which encodes Ig superfamily cell adhesion molecule (Sittaramane et al., 2009) (Gurung et al., submitted to Mechanisms of Development), and gpr125, which encodes an adhesion GPCR (Li et 
al., 2013), for FBM neuron migration. Moreover, mosaic analyses in zebrafish suggest that vang/2, fzd3a, celsr2, and scrib largely function non-cell autonomously during FBM neuron migration (Jessen et al., 2002; Wada et al., 2005; Wada et al., 2006), and potential roles for non-neuronal cell types in regulating FBM neuron migration cannot be ruled out (Sittaramane et al., 2013). Furthermore, a "collective mode" of migration which depends on interactions between FBM neurons may work in a PCP-independent manner to regulate migration (Davey et al., 2016; Walsh et al., 2011). Thus far, the roles of most genes in FBM neuron migration have been examined in isolation. A notable exception is Vangl2, which plays a central role in Wnt/PCP signaling. It has been demonstrated to exhibit genetic interactions with several non-PCP (Li et al., 2013; Sittaramane et al., 2009) and PCP genes (Carreira-Barbosa et al., 2003; Montcouquiol et al., 2003; Murdoch et al., 2014). However, some of these data were generated using morpholinos. Therefore, to address the concerns with morpholino-based studies, and to obtain a more comprehensive picture of the genetic interaction landscape, we performed an extensive pairwise analysis of several PCP and non-PCP genes. We compared the FBM neuron migration phenotypes of several combinations of double heterozygotes to those of their single heterozygote siblings. We found that vang/2 is rather unique in exhibiting genetic interactions with several PCP and non-PCP genes (vang/2; fzd3a and vang/2; cdh2, for example), while many of the other combinations ( $f z d 3 a$; scrb1 or fzd3a; $c d h 2$, for example) did not exhibit genetic interactions. These data highlight the central role of Vangl2 in Wnt/PCP signaling and suggest that it may 
play a key role in regulating the function of other PCP and non-PCP genes during FBM neuron migration.

\subsection{RESULTS}

\subsection{1 vang/2 genetically interacts with gpr125 for FBM neuron migration}

Migrating neurons likely regulate their behavior by interacting with neighboring cells. vang/2 is expressed ubiquitously during the period of FBM neuron migration and is required for and functions non-cell autonomously during FBM neuron migration (Bingham et al., 2002; Jessen et al., 2002; Sittaramane et al., 2013). Gpr125, an adhesion $\mathrm{G}$ protein-coupled receptor, is maternally provided and broadly expressed ( $\mathrm{Li}$ et al., 2013) in the zebrafish. However, morpholino-mediated knockdown of gpr125 rarely causes FBM neuron migration defects (Li et al., 2013). Consistent with this, we did not observe FBM neuron migration defects in MZgpr125 mutants (data not shown) suggesting that gpr125 does not have an essential function during FBM neuron migration. However, gpr125 has been shown to genetically interact with vangl2 during FBM neuron migration since injection of gpr125 MO enhances the weak FBM neuron migration phenotype of vang/2 heterozygotes (Li et al., 2013). Since these data were obtained from MO studies, we asked if the genetic interaction between vang/2 and gpr125 seen previously is preserved in mutants. To test this, we examined the offspring of MZgpr125-/- homozygous females and vangl2-/- homozygous mutant males, which 
are $100 \%$ double heterozygous embryos. The FBM neuron migration in double heterozygous embryos was binned into three categories based on the positioning of FBM neurons in different rhombomeres. 1) Normal migration indicates complete migration with more than $90 \%$ (estimated) of FBM neurons ending up in r6 and r7. 2) Partial block indicates reduced FBM neuron migration out of $r 4$ on one or both sides, with FBM neurons found throughout the migratory pathway from r4 to $r 7$, whereas 3 ) severe block indicates that a large majority of FBM neurons (estimated to be substantially greater than $50 \%$ ) remained in $r 4$ on both sides. FBM neuron migration defects were more severe and frequent in gpr125+/-; vang/2+/- double heterozygotes (Fig. 1D), compared to gpr125+/- or vangl2+/- embryos alone (Fig. 4.1B, C). FBM neurons migrated normally in $100 \%$ of gpr $125+/$ - embryos (Fig. 4.1B, E), and failed to migrate out of $\mathrm{r} 4$ in only $5 \%$ of vang/2+/- embryos (Fig. 4.1E). By contrast, FBM neurons failed to migrate out of $\mathrm{r} 4$ in $45 \%$ of gpr125+/-; vang/2+/- embryos (Fig. 4.1D, E). This genetic interaction between gpr125 and vang/2 using null mutant alleles is revealed only in sensitized genetic background, suggesting that gpr125 plays a definitive but minor role during FBM migration.

To further explore a role for gpr125 in FBM neuron migration, we examined another sensitized genetic background known to affect FBM neuron migration. Li et al., 2013 showed previously using gpr125 MO into the scribble1 (scrb1) mutant that gpr125 genetically interacts with scrb1, a PCP gene shown previously to regulate tangential migration of FBM neuron (Wada et al., 2005). We examined the offspring of MZgpr125-/- homozygous females and scrb1+/- heterozygous males, $50 \%$ of which are 
double heterozygous embryos. We found no embryos with severe FBM neuron migration defects, and the fraction of embryos with partial defects was comparable to that seen in gpr125+/- and scrb1+/- populations (data not shown). These data indicate that gpr125 and scrb1 do not interact genetically for FBM neuron migration and suggest that some morphant-associated phenotypes may not reflect true functions of gpr125 as seen previously between cntn2 and lamc1 (Gurung et al., submitted to Mechanisms of Development).

\subsection{2 vang/2 genetically interacts with cell adhesion gene $c d h 2$ and PCP gene fzd3a for FBM neuron migration}

Cadherin-2 (N-cadherin; Cdh2), a family of calcium-dependent cell adhesion molecules, has been implicated to play a role in various processes during neural development including neuron migration, axon elongation, pathfinding and fasciculation, target recognition and synaptogenesis (Suzuki and Takeichi, 2008). Cadherin-2 is expressed in both migrating FBMNs and the surrounding neuroepithelial cells (Hong and Brewster, 2006; Lele et al., 2002; Stockinger et al., 2011), and is required for FBM neuron migration (Rebman et al., 2016; Stockinger et al., 2011; Wanner and Prince, 2013). $f z d 3 a$, a PCP gene, is expressed throughout the hindbrain during the migration of FBM neurons and is required for and functions non-cell autonomously during FBM neuron migration (Wada et al., 2006). Given the unique nature of vang/2 in exhibiting genetic interactions with several non-PCP (Li et al., 2013; Sittaramane et al., 2009) and 
PCP genes (Carreira-Barbosa et al., 2003; Montcouquiol et al., 2003; Murdoch et al., 2014), we tested for genetic interaction between vang/2-cdh2 and vang/2-fzd3a. To test this, we examined the offspring of vang/2+/- heterozygous and $c d h 2+/-$ heterozygous fish, which are $50 \%$ vang/2+/- heterozygous embryos, out of which $50 \%$ are vang/2+/-; $c d h 2+/-$ double heterozygous embryos. FBM neuron migration defects were more severe and frequent in embryos obtained from crossing vang/2+/- heterozygous and $c d h 2+/-$ heterozygous fish, compared to embryos obtained from crossing vangl $2+/-$ heterozygous fish to wildtype fish which also generates $50 \%$ vang/2+/- heterozygous embryos. FBM neurons failed to migrate out of $\mathrm{r} 4$ in only $10 \%$ of embryos obtained from crossing vang/2+/- heterozygous fish to wildtype fish (Fig. 4.2). By contrast, FBM neurons failed to migrate out of $\mathrm{r} 4$ in around $50 \%$ of embryos obtained from crossing vang/2+/- heterozygous and cdh2+/- heterozygous fish (Fig. 4.2).

To test for possible genetic interactions between vang/2 and $f z d 3 a$, we examined the offspring of vangl $2+/$ - heterozygous and $f z d 3 a+/$ - heterozygous fish, which are $50 \%$ vang/2+/- heterozygous embryos, out of which $50 \%$ are vang/2+/-; fzd $3 a+/-$ double heterozygous. FBM neuron migration defects were more severe and frequent in embryos obtained from crossing vang/2+/- heterozygous and $f z d 3 a+/$ - heterozygous fish, compared to embryos obtained from crossing vang/2+/- heterozygous fish to wildtype fish generating $50 \%$ vang/2+/- heterozygous embryos. FBM neurons failed to migrate out of $\mathrm{r} 4$ in only $10 \%$ of embryos obtained from crossing vang/2+/heterozygous fish to wildtype fish (Fig. 4.2). By contrast, FBM neurons failed to migrate out of $\mathrm{r} 4$ in around $25 \%$ of embryos obtained from crossing vang/2+/- heterozygous and 
$c d h 2+/-$ heterozygous fish (Fig. 4.2). Together these data suggest that vang/2 genetically interacts with $c d h 2$ and fzd3a for FBM neuron migration.

\subsubsection{Other PCP and non-PCP genes do not exhibit genetic}

\section{interactions for FBM neuron migration}

Since vangl2 genetically interact with $c d h 2$ and $f z d 3 a$ for FBM neuron migration. We tested for genetic interaction between $c d h 2$ and fzd3a. To do this, we crossed $c d h 2+/-$ heterozygous and $f z d 3 a+/-$ heterozygous fish and examined the offspring which are $50 \% c d h 2+/$ - heterozygous embryos, out of which $50 \%$ are $c d h 2+/-; f z d 3 a+/$ - double heterozygous. FBM neuron defects seen in embryos obtained from crossing $c d h 2+/-$ heterozygous and fzd3a+/- heterozygous fish was comparable to defects seen in $c d h 2+/-$ heterozygotes suggesting that $c d h 2$ and $f z d 3 a$ do not interact with each other for FBM neuron migration. We also tested for genetic interaction between $f z d a 3 a$ and PCP gene (scrb1) and non-PCP genes (lamc1 and gpr125). However, we did not observe any pairwise genetic interaction for FBM neuron migration involving fzd3a, scrb1, lamc1 and gpr125. (Fig. 4.3A).

Contactin2 (Cntn2)/Transient Axonal Glycoprotein 1 (Tag1), a GPI-linked IgSFCAM, is implicated in the caudal migration of facial branchiomotor neuron (Sittaramane et al., 2009). Interestingly, cntn2 and vang/2 genetically interact during FBM neuron migration since injection of a suboptimal dose of cntn2 $\mathrm{MO}$ enhances the weak FBM neuron migration phenotype of vang/2 heterozygotes (Sittaramane et al., 2009). We 
tested for genetic interaction between chtn2 and several other PCP (vang/2, fzd3a and scrb1) and non-PCP genes (gpr125, cdh2 and lamc1) using genetic mutants. We detected interaction between cntn2 and vangl2 (see Chapter 3, figure 3.5) for FBM neuron migration. However, we did not observe any pairwise genetic interaction for FBM neuron migration involving cntn2, cdh2, fzd3a, lamc1, gpr125 and scrb1 (Fig. 4.3 and 4.4).

\subsection{DISCUSSION}

During the development of the nervous system, migrating neurons respond to several cues in the environment as well as the neighboring cells (Marin and Rubenstein, 2003). A large number of membrane associated molecules have been shown to play an important roles in guiding migrating neurons (Maness and Schachner, 2007). We performed an extensive pairwise analysis of several PCP (vang/2, fzd3a, scrb1) and non-PCP genes (cntn2, gpr125, cdh2, lamc1) that have been implicated in FBM neuron migration (Li et al., 2013; Rebman et al., 2016; Stockinger et al., 2011; Wada et al., 2005; Wada et al., 2006).

Vangl2, a core PCP molecule, has been shown to interact genetically with several PCP genes (Carreira-Barbosa et al., 2003; Montcouquiol et al., 2003; Murdoch et al., 2014) as well as non PCP (Li et al., 2013; Sittaramane et al., 2009) molecules. Using a combination of mutants and MOs, vang/2 has been previously shown to genetically interact with gpr125 for FBM neuron migration (Li et al., 2013). Interestingly, gpr125 has also been shown to genetically with scrb1, a PCP gene, for FBM neuron 
migration (Li et al., 2013). However, these data were generated using morpholinos. Therefore, to address the concerns with morpholino we only used genetic mutants to examine the genetic interaction. Here, using only mutants, we again observed genetic interaction between vang/2 and gpr125, but not between gpr125 and scrb1. These data indicate that a role for gpr125 in FBM neuron migration can be discerned in a sensitized vang/2+/- background but not in a sensitized scrb1+/- background. These data also suggest that some morphant-associated phenotypes may not reflect true functions of gpr125.

Given the unique nature of vang/2 in exhibiting genetic interactions we tested for genetic interaction between vang/2 and a cell adhesion gene $c d h 2$ and PCP gene $\mathrm{fzd} 3 \mathrm{a}$ for FBM neuron migration. We observed strong pairwise genetic interaction between vang/2; cdh2 as well as vangl2; fzd3a. However, we did not observe any interaction between $c d h 2$ and $f z d 3 a$ further suggesting the unique nature of vang/2 for FBM neuron migration.

Together, after careful analysis of pairwise genetic interaction between several PCP and non- PCP genes, we observed strong pairwise genetic interaction involving vangl2 with gpr125, cdh2, cntn2 (Chapter 3 ) and fzd3a but failed to see any pairwise genetic interactions not involving vang/2. Vangl2 appears to play a unique role in FBM neuron migration, distinct from the roles of other PCP and non-PCP genes. The connection between identified genetic interactions and the underlying molecular mechanisms is unclear. However, these genetic interactions suggest that these proteins may participate in a multi-component signaling complex at the FBM neuron cell 
membrane with membrane proteins on adjacent FBM neurons or non-neuronal cells (floor plate, neuroepithelial) to regulate neuronal migration. These data highlight the central role of Vangl2 in Wnt/PCP signaling and suggest that it may play a key role in regulating the function of other PCP and non-PCP genes during FBM neuron migration. On-going biochemical studies will determine if these proteins indeed are in a multiprotein complex. 


\subsection{FIGURES AND LEGENDS}

Figure 4.1: vang/2 genetically interacts with gpr125 for FBM neuron migration
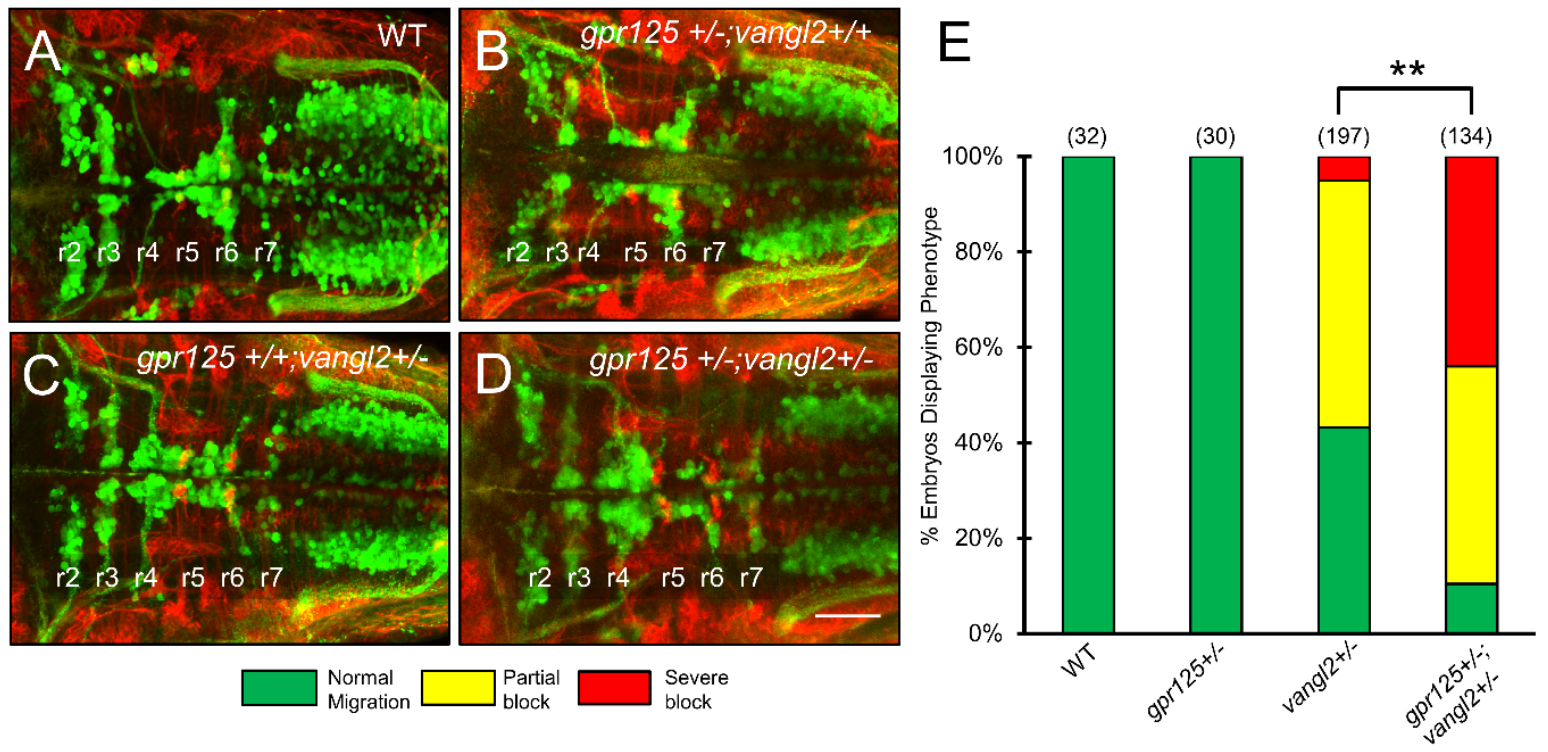
Figure 4.1: vang/2 genetically interacts with gpr125 for FBM neuron migration All panels show dorsal views of the hindbrain with anterior to the left. $T g$ (is/1:gfp) embryos were fixed at $48 \mathrm{hpf}$, and processed for immunohistochemistry with zn8 antibody (red) t0 label commissural neurons and axons at rhombomere boundaries (AD), and anti-GFP antibody (green) to label FBM neurons (A-D; arrowheads). (A) FBM neurons migrate normally in a control embryo. (B) FBM neurons migrate normally in a gpr125 heterozygous (gpr125+/-) embryo. (C) FBM neurons migrate poorly in a vang/2 heterozygous (vang/2+/-) embryo, with neurons located along the entire migratory pathway from r4 to r6. (D) FBM neurons fail to migrate out of $r 4$ in a gpr125; vang/2 double heterozygote (gpr125+/-; vang/2+/-). Scale bar in D, $50 \mu \mathrm{m}$ for A-D. (E) Quantification of genetic interaction data. Number in parenthesis denotes number of embryos. ${ }^{* *}$ Chi-square test at $p<0.001$; NS: not significant. Data are from 2 to 4 experiments. 
Figure 4.2: vang/2 genetically interacts with cell adhesion genes cdh2 and PCP gene fzd3a for FBM neuron migration

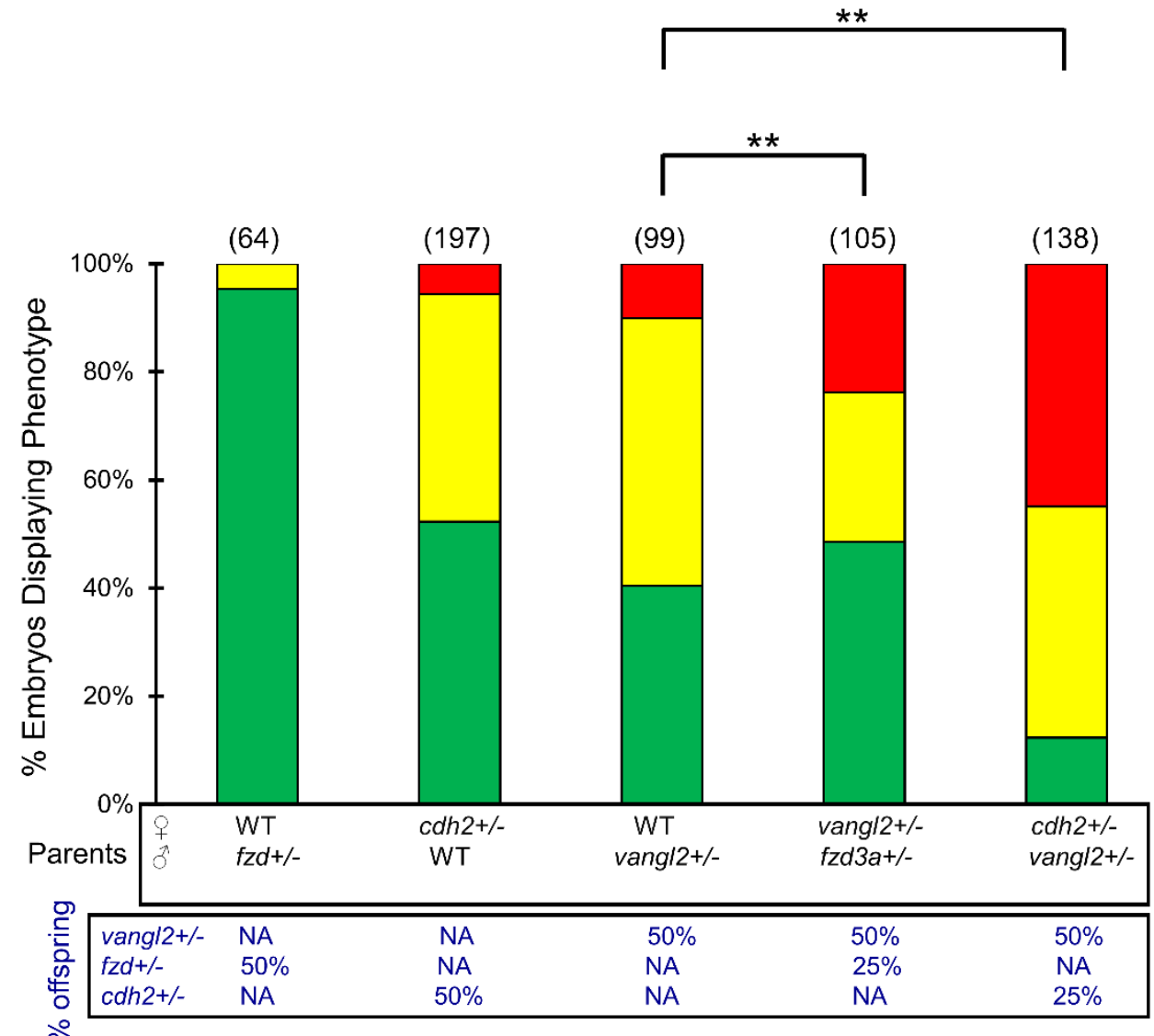


Figure 4.2: vang/2 genetically interacts with cell adhesion genes cdh2 and PCP gene fzd3a for FBM neuron migration

Quantification of genetic interaction data. Number in parenthesis denotes number of embryos. ${ }^{*}$ Chi-square test at $p<0.01$; NS: not significant. Data are from 2 to 4 experiments. 
Figure 4.3: Other PCP and non-PCP genes do not exhibit genetic interactions for FBM neuron migration

A
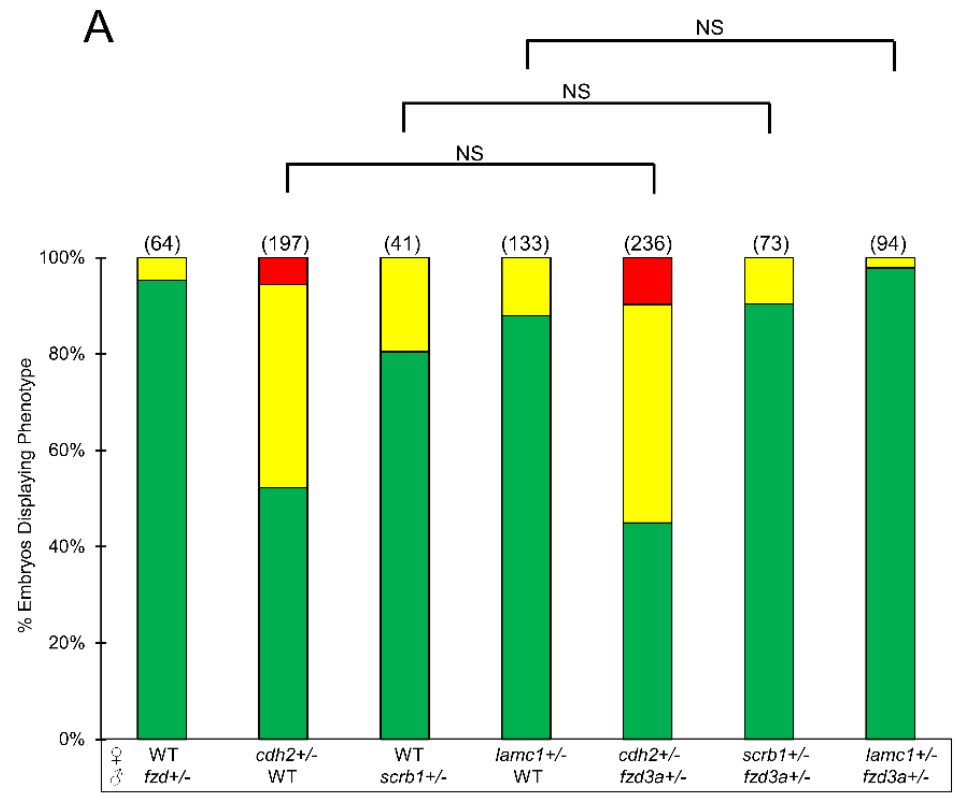

B
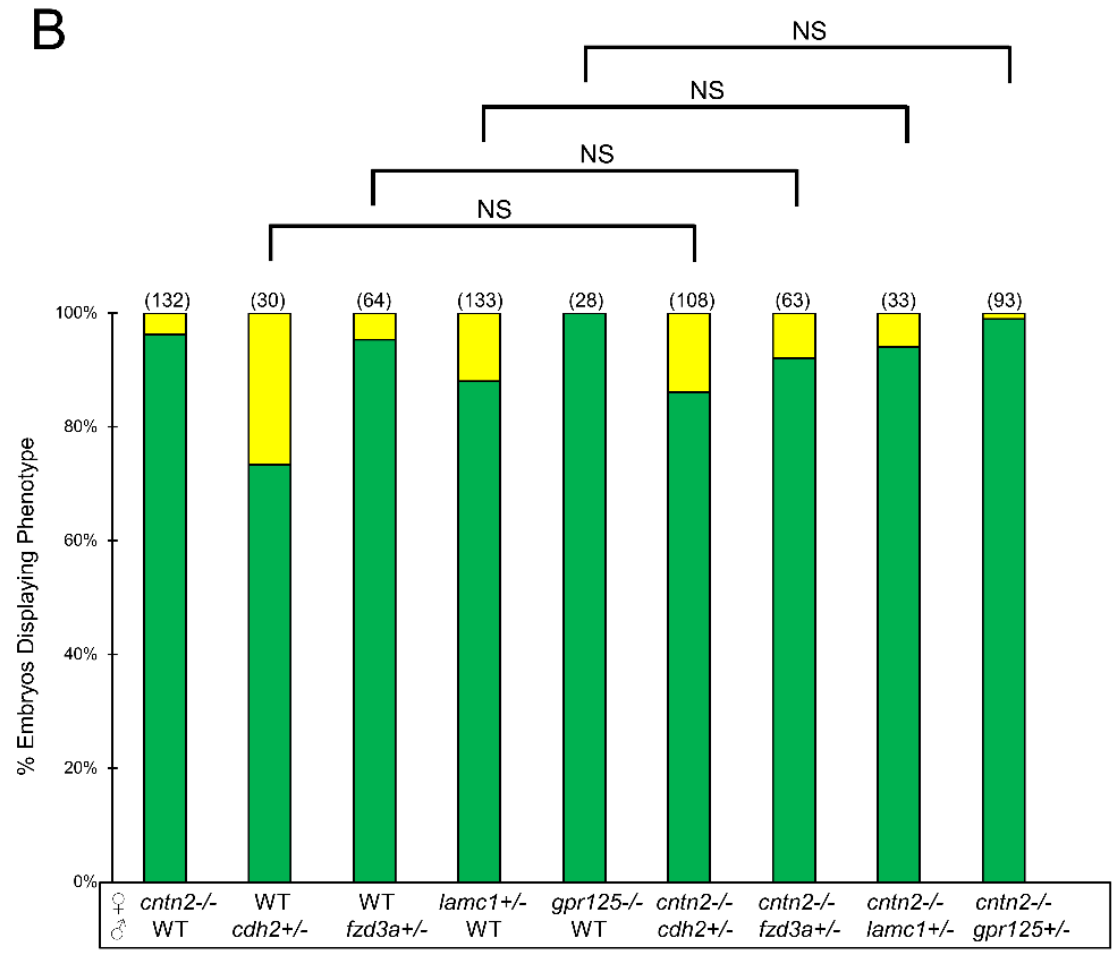
Figure 4.3: Other PCP and non-PCP genes do not exhibit genetic interactions for FBM neuron migration

Quantification of genetic interaction data. Number in parenthesis denotes number of embryos. Chi-square test. NS: not significant. Data are from 2 to 4 experiments. 
Figure 4.4: Summary of pairwise genetic interaction analyses

\begin{tabular}{|c|c|c|c|c|c|c|c|}
\hline & Vanql2 & Fzd3a & Scribble 1 & Gpr125 & Lamc1 & Cdh2 & Cntn2 \\
\hline Vangl2 & Yes & & & & & & \\
\hline Fzd3a & Yes & Yes & & & & & \\
\hline Scribble 1 & In progress & No & Yes & & & & \\
\hline Gpr125 & Yes & No & No & No & & & \\
\hline Lamc1 & No & No & In progress & In progress & Yes & & \\
\hline Cdh2 & Yes & No & In progress & In progress & In progress & Yes & \\
\hline Cntn2 & Yes & No & No & No & No & No & No \\
\hline
\end{tabular}


Figure 4.4: Summary of pairwise genetic interaction analyses

Pairwise genetic interaction tested for FBM neuron migration in this study. "Yes"

indicates that the genes interact genetically for FBM neuron migration whereas "No"

indicates no such genetic interaction was observed. 


\section{CHAPTER 5: CONCLUSIONS AND FUTURE DIRECTIONS}

In the preceding chapters, I investigated the roles of the Ig superfamily cell adhesion molecule Cntn2 in development and function of neural circuits in zebrafish.

First, I used CRISPR/Cas9 to generate cntn2 null mutants and demonstrated that cntn2 has a definitive but subtle role during FBM neuron migration (Chapter 3). Surprisingly, other phenotypes documented previously using morpholino-mediated cntn2 knockdown were not seen in cntn2 null mutants. My data showed that the dramatic morphant phenotypes were likely due to off-target effects of the cntn2 morpholino. Importantly, however, specific neuronal and behavioral defects were seen in cntn2 null mutants. Maternal-zygotic (MZ) cntn2 mutants exhibited axon fasciculation defects consistent with a role of Cntn2 in axon fasciculation. In addition, MZcntn2 mutant larvae exhibited aberrant touch responses and swimming, suggestive of defects in sensorimotor circuits, consistent with studies in mice. These data demonstrate distinct roles for zebrafish cntn2 in neuronal migration and axon fasciculation, and in the function of sensorimotor circuits. An exhaustive genetic interaction analysis of several planar cell polarity (PCP) and non-PCP genes (Chapter 4) demonstrated that vang/2 is rather unique in exhibiting genetic interactions with several PCP and non-PCP genes for FBM neuron migration. By contrast, all (fzd3a, scrb1, gpr125, lamc1, cdh2) other genes, PCP and non-PCP, involved in neuronal migration did not exhibit significant genetic interactions. These data suggest that vang/2 might be playing a central role in regulating function of many PCP and non-PCP genes for FBM neuron migration. I discuss below some questions and ideas for future experimentation. 


\subsection{Do genetic interactions reflect physical interactions}

\section{between membrane proteins during FBM neuron migration?}

We observed genetic interactions between vang/2 and three non-PCP genes cntn2, gpr125, and cdh2, and between vangl2 and fzd3a, a PCP gene, for FBM neuron migration. These data suggest that Vangl2 might play a central role in regulating the function of many PCP and non-PCP proteins. These genetic interactions suggest that these proteins may participate in a multi-component signaling complex at the FBM neuron cell membrane with membrane proteins on adjacent FBM neurons or nonneuronal cells (floor plate, neuroepithelial) to regulate neuronal migration. We can perform biochemical studies to determine if these proteins can indeed interact within a multi-protein complex. To do this, these proteins can be tagged with distinct epitopes (GFP, V5, Myc), and the proteins can be transiently expressed in zebrafish embryos by RNA injection. Co-immunoprecipitation analysis would reveal whether any specific protein pairs are present within a membrane complex, potentially interacting physically. We have previously expressed tagged full-length Vangl2, Cntn2, and Itga6 constructs separately in zebrafish embryos (Fig. 5.1). These analyses will be challenging because 1) all proteins being considered are membrane-associated, and coimmunoprecipitation analysis of membrane proteins is technically difficult, and 2) the proposed complexes are postulated to exist on a small group of migrating neurons and their neighboring cells in the zebrafish hindbrain, whereas these studies will employ protein-overexpressing cells isolated from the zebrafish at an earlier developmental stage. 


\subsection{What structural motif in Cntn2 is important for MLF axon}

\section{fasciculation?}

Cntn2 can act homophilically, as well as heterophilically, in cell adhesion and neurite outgrowth, and the immunoglobulin (Ig) and fibronectin (FN) domains have been implicated in both functions (Kunz et al., 2002; Pavlou et al., 2002). The FN domains and the two $\mathrm{N}$-terminal Ig domains have been implicated in cell adhesion while neurite outgrowth functions may be uniformly distributed amongst the lg and $\mathrm{FN}$ domains. We could test whether the Ig and FN domains have separable roles in MLF axon fasciculation by evaluating the abilities of various deletion constructs to rescue MLF defasciculation in MZcntn2 mutants. Variant proteins can be expressed specifically but transiently in MLF neurons by injecting Tol2-based plasmids containing cntn2 cDNAs under the control of the pitx2c element (Essner et al., 2000; Kikuta and Kawakami, 2009) (see Chapter 3, Fig. 3.4). If transgene expression is variable or rescue is inconsistent in these transient-expression studies, we can perform the rescue experiments by generating stable transgenic lines for the various Cntn2 deletion variants.

\subsection{Examine the function of genes misregulated in vang/2- deficient embryos}


The membrane protein Vangl2, a core component of Wnt/Planar Cell Polarity (PCP) pathway, functions independently of PCP signaling to regulate the caudal migration of facial branchiomotor (FBM) neurons in the vertebrate hindbrain (Glasco et al., 2012; Sittaramane et al., 2013). To gain insight into the underlying mechanisms, we used transcriptomic and proteomic analyses to identify genes or proteins whose expression levels are altered in hindbrains isolated from $18 \mathrm{hpf}$ Vangl2-deficient zebrafish embryos. Following bioinformatic analysis and manual curation of the top 300 misregulated genes, we generated a short list of roughly 50 candidate genes, and itga6a, Irrtm1, and sema6dl were among our top candidates for further analyses. These candidate genes are expressed in or around FBM neurons during the period of neuron migration (Fig. 5.2). Integrins are heterodimeric transmembrane receptors that mediate cell-adhesion (Hynes, 2002). Integrins interact with several extracellular and intracellular proteins to mediate their effects (Arnaout et al., 2007). Itga6 is a laminin binding integrin (Niculescu et al., 2011) . In zebrafish, itga6a is expressed broadly in the hindbrain. Interestingly, our preliminary data from morpholino studies that itga6a plays a role in FBM neuron migration (V. Sittaramane, S. Gurung, and A. Chandrasekhar, unpublished data). Leucine-rich repeats (LRR) are protein modules that are commonly involved in ligand receptor interactions or in cell adhesion (Kobe and Kajava, 2001).

Lrrtm1 is thought to be involved in brain development, neuronal connectivity, and synaptogenesis (Francks et al., 2007; Linhoff et al., 2009). Interestingly, Irrtm1 is expressed in FBM neurons. The semaphorin gene family contains a large number of transmembrane and secreted proteins and function as repulsive and attractive cues of 
axon guidance during development (Nakamura et al., 2000; Raper, 2000). Sema6dl is expressed in specific rhombomeres during the period of FBM neuron migration.

To directly examine the roles of these candidate genes in FBM neuron migration, I generated loss of function mutations in these genes using CRISPR-Cas9. I designed gRNAs (one target per gene) to generate frameshift mutations in several genes (itga6a, Irrtm1, sema6dl) at specific locations where small indels would likely cause loss of protein function. We have identified alleles for itga6a, Irrtm1, and sema6dl causing frameshifts that introduce early stop codons. Despite extensive analysis, I have not detected any defects in FBM neuron migration in zygotic or maternal zygotic mutants for any of these genes. However, other neuronal and behavioral phenotypes have not yet been characterized. Therefore, subtle phenotypes, as seen in MZcntn2 mutants, may be discovered in these mutants also. 


\subsection{FIGURES AND LEGENDS}

Figure 5.1: Tagged constructs and their detection by western blotting.

A

\begin{tabular}{|c|c|c|}
\hline Myc & Vangl2 & \\
\hline GFP & Cntn2 & \\
\hline & Itga6 & $\mathrm{HA}$ \\
\hline
\end{tabular}

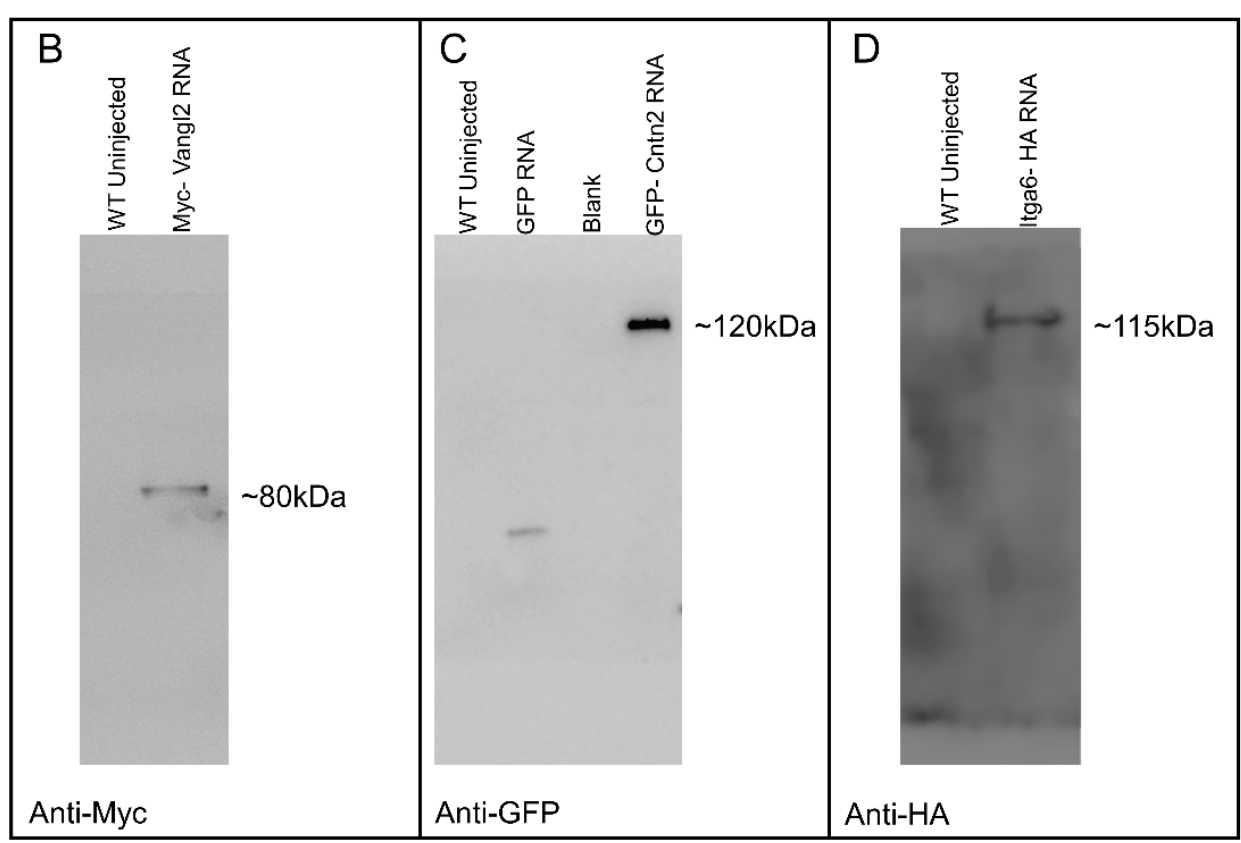


Figure 5.1: Tagged constructs and their detection by western blotting. Tagged constructs (A) Map of the tagged Vangl2, Cntn2, and Itga6 constructs. (B, C, D) Western blots to detect tagged Vangl2, Cntn2, and Itga6 proteins in lysates of $10 \mathrm{hpf}$ zebrafish embryos injected with the synthetic capped mRNAs. 
Figure 5.2: Expression pattern of candidate genes identified from transcriptomic analysis.

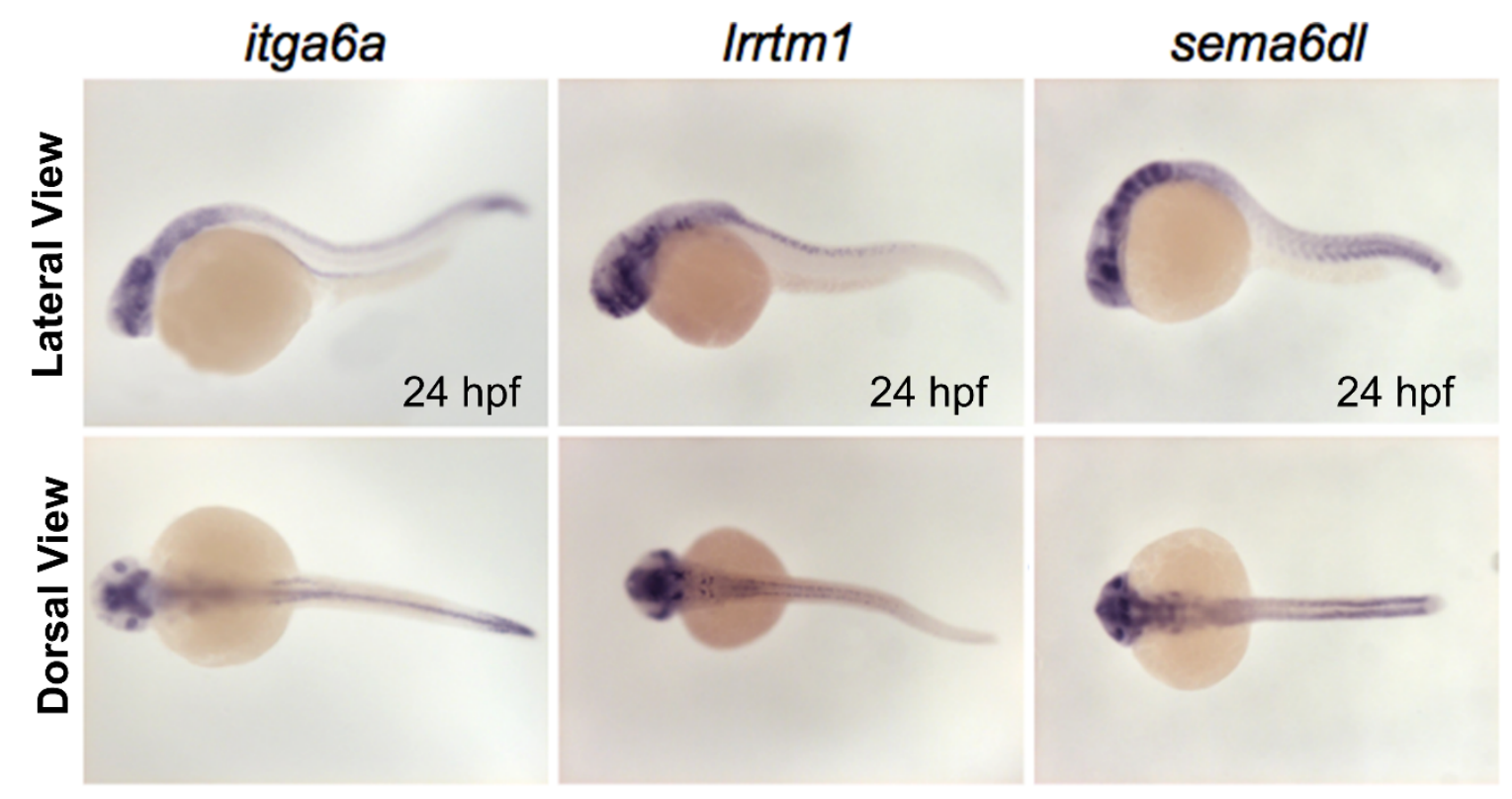


Figure 5.2: Expression pattern of candidate genes identified from transcriptomic analysis. In-situ hybridization performed at $24 \mathrm{hpf}$ embryos reveal that candidate genes are expressed in or around FBM neurons during the period of neuronal migration.

Itga6a is expressed in the lens, pronephric ducts, caudal somites and broadly in the hindbrain. Lrrtm1 is expressed in the spinal cord, retina proliferative zone, telencephalon and diencephalon. In the hindbrain, Irrtm1 is specifically expressed in the facial branchiomotor neuron. Sema6dl is expressed at the rhombomeres boundaries, somites, and the lens. In-situ images from zfin.org 


\subsection{TABLES}

Table 5.1 CRISPR/Cas9 target site and oligonucleotides used to make gRNA for itga6a, Irrtm1, and sema6dl

\begin{tabular}{|c|c|c|c|c|}
\hline Target gene & $\begin{array}{l}\text { Target gene } \\
\text { region }\end{array}$ & Target site & \multicolumn{2}{|c|}{ Oligonucleotides for annealing } \\
\hline itga6a & exon 5 & GGTCGGGGATGAAAACCGTCTGG & TAGGTCGGGGATGAAAACCGTC & AAACGACGGTTTTCATCCCCGA \\
\hline Irrtm1 & exon 1 & GGGCTGATACTGTGCTCCCTGGG & TAGGGCTGATACTGTGCTCCCT & AAACAGGGAGCACAGTATCAGC \\
\hline sema6dl & exon 3 & GGTATATTTGGTGAGTCTGAGGG & TAGGTATATTTGGTGAGTCTGA & AAACTCAGACTCACCAAATATA \\
\hline
\end{tabular}

Sequences of the genomic target sites and oligonucleotides for making the gRNA are shown. Sequence present in the gRNAs are in bold. PAM is underlined.

Table 5.2 Summary of CRISPR/Cas9 generated alleles of itga6a, Irrtm1, and sema6dl

\begin{tabular}{|c|c|c|c|}
\hline Gene & Allele 1 & Allele 2 & Allele 3 \\
\hline itga6a & $-11 \mathrm{bp}$ (premature stop) & $-4 \mathrm{bp}$ (premature stop) & +7 (premature stop) \\
\hline Irrtm1 & $-4 \mathrm{bp}$ (premature stop) & $+4 \mathrm{bp}$ (premature stop) & $-20 \mathrm{bp}$ (premature stop) \\
\hline sema6dl & $-9 \mathrm{bp}$ (in-frame deletion) & $-6 \mathrm{bp}$ (in-frame deletion) & $-11 \mathrm{bp}$ (premature stop) \\
\hline
\end{tabular}




\section{CHAPTER 6: A NOVEL GENETIC CELL ABLATION}

\section{TECHNOLOGY IN ZEBRAFISH}

Suman Gurung ${ }^{1}$, Marina M. Hanson ${ }^{2}$, Devynn Hummel ${ }^{1}$, Miriam Hankins ${ }^{2}$, Xuebin Qin ${ }^{3}$,

Elizabeth C. Bryda ${ }^{2}$, and Anand Chandrasekhar ${ }^{1}$

1, Division of Biological Sciences and Bond Life Sciences Center, University of

Missouri, Columbia, MO 65211, USA

2, Department of Veterinary Pathobiology, College of Veterinary Medicine, University of

Missouri, Columbia, MO 65211, USA

3, Department of Neuroscience, Temple University School of Medicine, Philadelphia,

PA 19140, USA

Corresponding Author:

Dr. Anand Chandrasekhar

Division of Biological Sciences

Room 340D Bond Life Sciences Center

1201 Rollins St

University of Missouri, Columbia, MO 65211-7310

Tel: (573) 882-5166; Fax: (573) 884-9676

Email: AnandC@missouri.edu

Running Title: Genetic cell ablation technology in zebrafish

Keywords: zebrafish; cell ablation; hCD59; intermedilysin; zCREST, genetic; inducible

$\underline{\text { To be submitted to Zebrafish }}$ 


\subsection{ABSTRACT}

Targeted cell ablation is a powerful approach to for investigating cellular functions, tissue regeneration, developmental processes and pathogenesis of human disease. Several methods have been developed to accomplish cell ablation, however, current methodologies have limitations including relatively slow onset of cell ablation. Intermedilysin (ILY) is a cytolytic pore-forming toxin secreted by Streptococcus intermedius that exclusively lyse human cells by specifically binding to human CD59 receptor. In this study, we have optimized physiological parameters for the application of the hCD59-ILY system in zebrafish. Embryos injected with RNA encoding wildtype hCD59 exhibited rapid tissue lysis upon ILY treatment. However, embryos injected with RNA encoding mutant hCD59 RNA, which lacks the ILY binding site, did not exhibit any cell lysis, demonstrating the specificity of the hCD59-ILY system. In addition, to validate the hCD59-ILY system in an intact embryo expressing hCD59 in specific neural cell types, we generated transgenic fish expressing hCD59 in branchiomotor neurons using zCREST promoter. ILY treatment of hCD59-expressing neurons plated in culture from this transgenic line resulted in rapid lysis, definitively demonstrating that zebrafish cells are susceptible to ablation using this system. We are currently testing the efficacy of the hCD59-ILY system to mediate rapid ablation of hCD59-expressing cells in intact embryos. 


\subsection{INTRODUCTION}

Cell-specific ablation is a powerful tool for understanding cell lineage, cell function, developmental processes and disease mechanisms (Buch et al., 2005; Lahl et al., 2007; Luquet et al., 2005; Saito et al., 2001). Physical, chemical, and genetic methods have been developed to ablate cells ablation in various model organisms (Curado et al., 2007; Lewandoski, 2001; McGuire et al., 2004). However, current methodologies have limitations including lack of cell-type specificity, off-target effects, narrow pharmacological windows, and relatively slow onset of cell death.

Zebrafish is an excellent model system for studying development and disease. Due to their optical transparency, zebrafish embryos have proven to be an excellent experimental system for analyzing dynamic cellular processes including cell migration, axon guidance, and myelination (Bingham et al., 2002; Pogoda et al., 2006; Wolman et al., 2008), which involve intercellular interactions and signaling. Due to its regenerative abilities, zebrafish are also an excellent model system for understanding the mechanisms underlying tissue regeneration. To study the roles of specific cell types and interactions during development and regeneration, various cell ablation methods employing physical, chemical and genetic approaches have been developed. Physical ablation which includes laser ablation or tissue removal by surgery allows both temporal and spatial control (Gahtan and Baier, 2004; Yang et al., 2004). However, these methods are crude and often lack specificity, hence difficult to replicate. Furthermore, these methods are labor-intensive, requiring manual manipulation of individual samples during laser ablation or surgery. While chemical ablation such as 2-butoxyethanol (BE) 
for erythrocyte ablation (Koshkaryev et al., 2003) can be less labor-intensive and timeconsuming than physical ablation, it is difficult to induce ablation efficiently without toxic off-target effects (Dornfest et al., 1990; Klinken et al., 1987).

Genetically-mediated ablation involves the expression of toxin-producing enzymes or toxin-specific receptors in the cells being targeted for ablation. For example, the expression of diphtheria toxin A (DTA) using cell type-specific promoters can induce tissue-specific ablation (Kurita et al., 2003; Lee et al., 2015). However, off-target effects, as well as toxicity, have been observed. In addition, due to the high potency of DTA, leakiness of the minimal promoter can prevent the generation of transgenic zebrafish lines (Wan et al., 2006). Another genetic cell ablation system using bacterial Nitroreductase (NTR) and a nontoxic prodrug Metronidazole (Mtz), has been widely used in zebrafish to ablate cells with spatial and temporal control (Ariga et al., 2010; Chen et al., 2011; Zhao et al., 2009). Within cells expressing Nitroreductase, the enzyme converts the prodrug Mtz into a cytotoxic DNA cross-linking agent that induces cell death by apoptosis (Knox et al., 1988) (Curado et al., 2008). While the NTR/Mtz method offers clear advantages over physical and chemical ablation approaches, it can have bystander effects on neighboring cells when dying cells release the toxic drug (Grohmann et al., 2009). Another caveat of this method is the relatively slow onset of cell death. Therefore, it would be highly desirable to develop a genetic approach for targeted, rapid cell ablation.

We describe a novel cell ablation system for zebrafish that utilizes the human CD59 receptor (hCD59), and the bacterial toxin intermedilysin (ILY) (Hu et al., 2008). 
hCD59 is a widely expressed cell surface 128 amino acid glycoprotein linked to the membrane through a glycosylphosphatidylinositol (GPI) anchor (Wickham et al., 2011). ILY is approximately $50 \mathrm{kDa}$ toxin secreted by a Gram-positive pathogenic bacterium, Streptococcus intermedius, and is a member of cholesterol-dependent cytolysins (CDCs). ILY selectively binds to hCD59, but not to cognate receptors from other vertebrates (Nagamune et al., 1996), and induces pore formation and cell lysis. In mice, hCD59 has been specifically expressed particular cell types, which were lysed (ablated) rapidly following ILY treatment (Feng et al., 2016; Hu et al., 2008). We have tested the effectiveness of the hCD59-ILY cell ablation system in zebrafish.

\subsection{MATERIALS AND METHODS:}

\subsubsection{Zebrafish husbandry and maintenance}

Zebrafish (Danio rerio) were maintained following standard protocols and University of Missouri ACUC guidelines as described previously (Sittaramane et al., 2013; Westerfield, 1995). Embryos were grown in E3 medium at $28.5^{\circ} \mathrm{C}$, staged by hours post fertilization (hpf) (Kimmel et al., 1995), and embryo age was verified at somitogenesis stages.

\subsubsection{Testing for salt tolerance}

For salt tolerance testing, embryos were dechorinated at $10 \mathrm{hpf}$, and exposed to a range of $\mathrm{NaCl}$ concentrations in $\mathrm{E} 3$ medium. Different $\mathrm{NaCl}$ concentrations were 
generated by dilution using a $5 \mathrm{Molar} \mathrm{NaCl}$ stock solution. Mortality was assessed after overnight ( 14 hours) incubation.

\subsubsection{Assessing the effects of ILY treatment}

Wildtype embryos were dechorinated at $23 \mathrm{hpf}$ and incubated in E3 medium for one hour at $28.5^{\circ} \mathrm{C}$. At $24 \mathrm{hpf}$, embryos were placed in the wells of a 12-well plate (20 embryos per well) containing E3 medium with $90 \mathrm{mM} \mathrm{NaCl}$. Embryos were incubated at $37^{\circ} \mathrm{C}$ for 20 minutes to acclimate. Next, ILY was added to each well to a final concentration of 3-6 $\mathrm{ng} / \mu \mathrm{L}$, followed by incubation at $37^{\circ} \mathrm{C}$. Embryo mortality (and tissue integrity) was assessed after 1 hour of incubation.

\subsubsection{Intermedilysin (ILY)}

ILY is a member of cholesterol-dependent cytolysins (CDCs) is composed of four structural domains (D1-D4) and is approximately $50 \mathrm{kDa}$ (Nagamune et al., 1996; Ohkura et al., 2012; Tabata et al., 2014). ILY is secreted by the Gram-positive pathogenic bacterium, Streptococcus intermedius. Domain 1 is at the top of the molecule which acts as a foundation for other domains. Domain 2 connects domain 1 and 4. Domain 3 packs against domain 2 and forms the transmembrane $\beta$-barrel pore. Domain 4, which contains three membrane binding loops, contribute to the human specificity of ILY (Giddings et al., 2003; Giddings et al., 2004). ILY binding to CD59 triggers a series of conformational changes in ILY leading to its membrane 
oligomerization which causes pore formation (Soltani et al., 2007). ILY is relatively stable between $0^{\circ} \mathrm{C}$ and $30^{\circ} \mathrm{C}$ but starts to lose its activity above $40^{\circ} \mathrm{C}$ with a complete loss at temperatures above $50^{\circ} \mathrm{C}$ (Nagamune et al., 1996).

\subsection{5 hCD59 constructs and RNA synthesis}

CD59, a small protein of approximately 77 amino acids and weighing $20 \mathrm{kDa}$, is ubiquitously expressed in all human tissues (Davies et al., 1989). The pCS2P+ plasmid was obtained from Addgene (\#17095). In-fusion HD Cloning system (Clontech) was used to insert the ZsGreen-P2A-hCD59 fragment into the pCS2 backbone. The resulting construct is referred as wildtype (WT) $h C D 59$ from hereon. Mutant (Mut) $h C D 59$ was generated by modifying the pCS2- WT hCD59 plasmid to delete the implicated ILY-binding domain of hCD59 (Hughes et al., 2009). The plasmids were linearized with enzyme Pvull (NEB), and capped WT $h C D 59$ and mut $h C D 59$ mRNA was synthesized using the mMessage mMachine SP6 Kit (Ambion) following manufacturer's protocol. The mRNA was checked for purity and size by gel electrophoresis, and concentration was determined using a Nanodrop spectrophotometer (Thermo Scientific).

\subsubsection{Validation of $h C D 59$-mediated cell ablation in transient} expression assay 
Wildtype nontransgenic embryos at the 1-2 cell stage were microinjected with wildtype $h C D 59$, mutant $h C D 59$, or GFP mRNA. The injection volume (and mRNA dose) was calibrated by measuring the diameter of droplets injected into mineral oil placed on a slide containing an etched micrometer scale. Approximately 600-1200 pg mRNA was injected into each embryo with an MPPI-2 pressure injector (Applied Scientific Inc.). Uninjected embryos and GFP mRNA-injected embryos from the same clutch served as controls. Injected embryos were examined for strong ZsGreen expression. Embryos with ubiquitous and high level of ZsGreen expression were dechorinated at $23 \mathrm{hpf}$ and incubated in E3 medium for one hour at $28.5^{\circ} \mathrm{C}$. At $24 \mathrm{hpf}$, embryos were placed in wells of 12 well plate (10 embryos per well) containing E3 medium with $90 \mathrm{mM} \mathrm{NaCl}$, treated with ILY $(4 \mathrm{ng} / \mathrm{\mu l})$ or saline (control), and incubated at $37^{\circ} \mathrm{C}$. Embryo health and mortality were assessed at $1 \mathrm{~h}$ and $24 \mathrm{~h}$ after ILY addition.

\subsubsection{Generation of zCREST:hCD59 transgenic zebrafish}

We generated a transgenic line $\operatorname{Tg}(z C R E S T: Z s G r e e n-h C D 59)$, expressing ZsGreen and hCD59 in branchiomotor neurons using Tol2 transposon-based transgenesis. The ZsGreen-P2A-hCD59 fragment was released from the $\mathrm{pCS} 2-\mathrm{WT}$ $h C D 59$ vector and inserted into pME-MCS to generate pME-ZsGreen-P2A-hCD59 vector. The Tol2 kit was kindly provided by Kristen Kwan and Chi-Bin Chien, University of Utah (Kwan et al., 2007). We used p5E-zCREST, pME-ZsGreen-P2A-hCD59 vector, p3E-poly(A) and pDestTol2CG2 in a multiple cloning LR reaction (Kwan et al., 2007) to establish the final Tol2 vector for injecting into fertilized eggs (Fig. 6.4). $50 \mathrm{pg}$ of the 
Tol2-hCD59 vector was injected with $25 \mathrm{pg}$ of Tol2 transposase mRNA into each fertilized egg obtained from crosses of wildtype nontransgenic fish. Injected embryos were screened at 24-36 hpf for GFP expression in the heart (Tol2 marker) and for ZsGreen expression in branchiomotor neurons and raised to adulthood. F1 progeny were screened for germline transmission of the zCREST:ZsGreen-hCD59 transgene, and ZsGreen-expressing F2 embryos were raised to adulthood to establish the transgenic line.

\subsubsection{Primary culture of zebrafish embryonic neurons}

We employed a recently established protocol (Sassen et al., 2017) with minor modifications. Embryos ( 200) were anesthetized with tricaine at 2-3 dpf, transferred to a $50 \mathrm{ml}$ Falcon tube, and washed twice with 30\% Danieau buffer. Embryos were resuspended in deyolking buffer 50\% Ginzburg Fish Ringer (Whitlock and Westerfield, 2000) without calcium: $55 \mathrm{mM} \mathrm{NaCl}, 1.8 \mathrm{mM} \mathrm{KCl}, 1.25 \mathrm{mMNaHCO}$ ), and deyolked by repeated trituration with $1 \mathrm{ml}$-pipette tip and brief vortexing. Deyolked embryos were pelleted at $300 \mathrm{xg}$ for $1 \mathrm{~min}$, resuspended in $1 \mathrm{ml}$ of deyolking buffer, and transferred to a sterile cell strainer (40 $\mu \mathrm{m}$, Cat \# 22-363-547, ThermoFisher) fitted for $50 \mathrm{ml}$ Falcon tubes. The strainer containing deyolked embryos was dipped in $70 \%$ ethanol for 5 seconds to sterilize the tissue and submerged into culture medium (Leibovitz's L-15 medium supplemented with $10 \%(\mathrm{v} / \mathrm{v})$ filtered bovine serum and 10,000 units $/ \mathrm{ml}$ PenStrep). Embryos were transferred to a sterile $1.5 \mathrm{ml}$ tube in culture medium (1 $\mathrm{ml})$, and collagenase (Cat \# 234155, MilliporeSigma) prepared in culture medium was added 
to a final concentration of $4 \mathrm{mg} / \mathrm{ml}$ to obtain dissociated cells. The embryos were incubated on a rotator for 3 hours at room temperature with a brief vortex every 30 minutes. The dissociated embryo mixture was filtered through a sterile cell strainer into a $50 \mathrm{ml}$ Falcon tube to remove debris, and washed with $1 \mathrm{ml}$ of culture medium, and cells were pelleted by centrifugation ( $250 \mathrm{xg}, 5 \mathrm{~min})$. The cell pellet was gently resuspended in $1 \mathrm{ml}$ of culture medium, plated on poly-L-lysine coated plates (Cat \# 08$774-124$, ThermoFisher) and incubated at $28^{\circ} \mathrm{C}$ in a cell culture incubator without $\mathrm{CO}_{2}$. The culture medium was replaced every 6 hours for first 12 hours, and every 12 hours thereafter. Cell density and viability were monitored using a Nikon TMS inverted scope.

\subsubsection{Ablation in primary cell culture}

Embryos from $T g(z C R E S T: Z s G r e e n-h C D 59)$ crosses were screened for ZsGreen expression in branchiomotor neurons $T g(i s / 1: g f p)$ embryos (Higashijima et al., 2000), which express GFP in branchiomotor neurons, were used as controls. Primary cell cultures were established from ZsGreen- and GFP- expressing embryos, and ablation experiments were performed after 24 hours of plating. For imaging cells during the ablation process, a Zeiss Axiovert 200M inverted microscope with an environmental temperature control chamber was used with the chamber temperature set to $37^{\circ} \mathrm{C}$. The cell culture dish was incubated in the chamber for 30 min to gradually bring the cultured cells up to $37^{\circ} \mathrm{C}$. After locating small clusters or isolated ZsGreen- or GFP-expressing neurons, ILY was added to the culture medium, and the fluorescent cells were monitored for $30 \mathrm{~min}$. 


\subsection{RESULTS}

\subsubsection{Establishing optimal conditions for testing the efficacy of ILY treatment in zebrafish embryos}

Previous studies have shown that concentrations of $\mathrm{NaCl}$ below $50 \mathrm{mM}$ completely abolish the cytolytic activity of ILY at $37^{\circ} \mathrm{C}$ (Nagamune et al., 1996). To evaluate the feasibility of ILY-mediated ablation in zebrafish, we first tested the tolerance of zebrafish embryos to elevated $\mathrm{NaCl}$ and temperature levels. Dechorionated embryos were incubated in $\mathrm{E} 3$ medium from $10 \mathrm{hpf}$ at $28.5^{\circ} \mathrm{C}$ in $\mathrm{NaCl}$ concentrations ranging from $20 \mathrm{mM}$ to $140 \mathrm{mM}$. Untreated (E3 only) embryos and embryos treated with up to $90 \mathrm{mM} \mathrm{NaCl}$ did not display any gross morphological defects or mortality (Fig. 6 . 1A). However, embryos treated with $\mathrm{NaCl}$ concentrations greater than $90 \mathrm{mM}$ exhibited significant mortality with only $20 \%$ survival at $140 \mathrm{mM} \mathrm{NaCl}$ concentration (Fig. 6.1A). This result suggests that the ILY treatments can be performed in $90 \mathrm{mM} \mathrm{NaCl}$, which although not optimal, still supports ILY activity (Nagamune et al., 1996).

Next, to determine their tolerance to $37^{\circ} \mathrm{C}$, the optimal temperature for ILY activity, zebrafish embryos at $24 \mathrm{hpf}$ were incubated for 1 hour at $28^{\circ} \mathrm{C}$ and $37^{\circ} \mathrm{C}$. No gross morphological differences or mortality were observed between the two groups and all embryos survived (data not shown), indicating that brief incubations at $37^{\circ} \mathrm{C}$ to induce cell death are not detrimental.

Finally, to test whether ILY itself has any detrimental effect, nontransgenic embryos were immersed in varying concentrations of ILY at elevated $\mathrm{NaCl}$ 
concentration $(90 \mathrm{mM})$ and temperature $\left(37^{\circ} \mathrm{C}\right)$. In mammalian cells expressing hCD59, ILY induces rapid cell lysis (Hu et al., 2008). PBS-treated (control) embryos and embryos treated for 1 hour with up to $4 \mathrm{ng} / \mathrm{\mu l}$ of ILY did not exhibit any adverse effects or mortality (Fig. 6.1B). However, embryos treated with 5-6 $\mathrm{ng} / \mu \mathrm{l}$ of ILY became morbid, with extensive embryo death (Fig. 6.1B), suggesting that ILY (or a breakdown product or other contaminant in the preparation) can have non-specific detrimental effects on zebrafish embryos at concentrations above $4 \mathrm{ng} / \mu \mathrm{l}$. Together, these data establish that the hCD59-ILY system can be tested for feasibility in zebrafish within a defined range of $\mathrm{NaCl}$ and ILY concentrations.

\subsubsection{ILY induces mortality in hCD59-expressing embryos, and requires the ILY-binding site}

To rapidly assess the efficacy of ILY-mediated cell ablation in zebrafish, we tested the ability of ILY to kill cells in embryos expressing hCD59 transiently but ubiquitously following injection of synthetic mRNA. Uninjected or GFP mRNA-injected embryos were used as controls. Embryos injected with either $h C D 59$ or GFP mRNAs were positive for ZsGreen and GFP, respectively (Fig. 6.2B; data not shown). Following ILY addition, control embryos remained healthy for several hours (Fig. 6.2C, F, G), with no visible effect on any tissues. In sharp contrast, about $25 \%$ of WT hCD59-expressing embryos had dark, condensed yolk sacs within 1 hour of ILY exposure (Fig. 6.2D, F). At higher magnification, WT hCD59 expressing embryos showed vacuolation in the trunk 
(Fig. 6.3). Moreover, all (100\%) of the WT hCD59-expressing embryos were dead after 24 hours of ILY treatment, whereas uninjected embryos remained completely healthy (Fig. 6.2G). To determine whether ILY was inducing specific effects, we tested the effects of ILY treatment on embryos expressing a mutant hCD59 lacking the ILY-binding domain (hCD59 $\triangle \mathrm{IBD}$; Fig. 6.2A). Embryos expressing hCD59 $\triangle \mathrm{IBD}$ remained completely healthy for 24 hours following ILY addition (Fig. 6.2E-G), suggesting strongly that ILY is specifically binding to hCD59 to induce tissue breakdown, leading to embryo mortality.

\subsubsection{ILY-induced ablation of a specific cell type in transgenic}

\section{embryos}

To test the efficacy of the hCD59/ILY system for spatiotemporally-controlled cell ablation in zebrafish, we used the Tol2 system to generate a stable transgenic line in which ZsGreen and hCD59 are specifically expressed in branchiomotor neurons using the zCREST enhancer ((Uemura et al., 2005); Fig. 6.4A)). The generated transgenic line stably expresses ZsGreen-hCD59 in branchiomotor neurons (Fig. 6.4B). Initially we attempted to ablate ZsGreen-hCD59 expressing branchiomotor neurons by ILY treatment in transgenic embryos. However, we did not see any ablation of branchiomotor neurons after ILY treatment in intact embryos (Fig. 6.5).

Since it was unclear whether hCD59 cells within the intact embryo were being ablated following ILY treatment, we tested whether ILY could ablate hCD59-expressing cultured neurons. We cultured cells isolated from $\operatorname{Tg}(z C R E S T: Z s G r e e n-h C D 59)$ embryos, which express ZsGreen and hCD59 specifically in branchiomotor neurons 
(See Materials and Methods). Cells from $\operatorname{Tg}($ is/1:gfp) embryos, which express GFP in branchiomotor neurons, were used as controls. Cultured fluorescent cells expressing GFP or ZsGreen could frequently be identified as neurons due to the presence of neurites or axonal processes. To detect dying cells unambiguously, only isolated fluorescent cells and small cell clusters ( $<5$ cells) were analyzed. Within 5 min of ILY addition, there was a reduction of ZsGreen fluorescence intensity in these hCD59expressing cells (Fig. 6.6B'). By 30 min, ZsGreen fluorescence intensity was greatly reduced or completely lost in the majority of cells analyzed (Fig. 6.6B", C). In contrast, GFP fluorescence intensity in control neurons cultured from $\mathrm{Tg}$ (islet1:gfp) embryos remained unchanged up to $30 \mathrm{~min}$ after ILY addition (Fig. 6.6A' and 6.6A"). These data demonstrate that the ILY effectively ablated cultured zebrafish neurons expressing hCD59, while having no effect on cells not expressing hCD59. The data also suggest that the ILY-hCD59 system is working as expected in tissues within live embryos.

\subsection{DISCUSSION}

A large number of cell ablation techniques have been developed which have been instrumental in understanding the role of a specific cell lineage or tissue in developmental or physiological processes (Buch et al., 2005; Lahl et al., 2007; Luquet et al., 2005; Saito et al., 2001). Genetic cell ablation methods have proven to be superior over physical and chemical ablation methods which are labor intensive, timeconsuming, and lack specificity. Current genetic cell ablation methods include expression of herpes simplex virus 1 thymidine kinase (HSVtk), bacterial nitroreductase, 
and the diphtheria toxin (DT) receptor coupled with transgenic or viral delivery strategies. However, current genetic methodologies have limitations including lack of cell-type specificity, off-target effects, narrow pharmacological windows, and relatively slow onset of cell death.

To obviate these limitations, in this study, we aimed to provide proof of concept that ILY administration to ablate cells expressing hCD59 provides a sensitive and specific tool for cell ablation in zebrafish. The potent cytotoxin ILY, secreted by Streptococcus intermedius (SI), binds exclusively to the human cell membrane protein CD59 (hCD59) but not to CD59 of any other species tested to date (Nagamune et al., 1996). Once bound, ILY rapidly (within seconds) lyses the cells likely without creating an inflammatory reaction and without significant off-target effects. hCD59/ILY system has been recently proven successful at lysing a variety of cell types (e.g., erythrocytes and endothelia cells) in mouse transgenic models (Hu et al., 2008).

Zebrafish have become a favorite organism for studying vertebrate development. Zebrafish have large progeny clutch, breed all year, and are easily maintained. They also have transparent embryos that develop outside the mother allowing exquisite visual inspection of cell types for analysis of dynamic cellular processes. In addition, zebrafish embryo develops rapidly forming most of its tissues and organ primordia by 24 hours after fertilization. Therefore, in zebrafish, there is a great need for a rapid cell ablation system. In zebrafish, targeted conditional cell ablation system by combining the expression of bacterial Nitroreductase (NTR) enzyme in a tissue-specific manner, with treatment with the prodrug Metronidazole (Mtz), which is converted to a cytotoxic DNA 
crosslinking agent by the enzymatic activity of NTR have been developed (Curado et al., 2008). The NTR/Mtz system is spatially controllable and strictly confined to the target cell population, temporally inducible, germline transmissible, and reversible (Curado et al., 2007). However, to achieve ablation using this system, fish must be treated with Mtz at near-toxic concentrations for 24 hours. In addition, a delayed continuous cell loss have been observed after Mtz removal (Mathias et al., 2014). Therefore, given this limitation of NTR/Mtz ablation system involving slow onset of cell death, we aimed to develop a rapid ablation technique and demonstrate the wide application and utility of the hCD59-ILY cell ablation system in zebrafish. In this study, using transient RNA injection and development of stable zebrafish transgenic line, we have shown that the hCD59/ILY system can be used to effectively ablate cells in zebrafish.

Since the system was optimized for mammals with different physiology than fish, our initial work involved finding optimal physiological parameters, optimal route of administration, and optimal dose. Since zebrafish embryos can easily uptake drugs in water, we employed immersion which is a non-invasive commonly used route of administering drugs in fish (Pisharath and Parsons, 2009). Moreover, this method also offers temporal control because the drugs can be added to and removed from the fish water at will (Curado et al., 2007).

Previous study has shown that the activity of ILY begins to decrease at temperatures below $20^{\circ} \mathrm{C}$ and is completely lost at salt concentrations less than $50 \mathrm{mM}$ $\mathrm{NaCl}$ (Nagamune et al., 1996). Since zebrafish embryos are kept in E3 medium 
(containing only $5 \mathrm{mM}$ of $\mathrm{NaCl}$ ) at $28.5^{\circ} \mathrm{C}$, we tested salt and temperature tolerance of zebrafish embryos. No gross morphological differences or mortality were observed when zebrafish embryos were incubated at $37^{\circ} \mathrm{C}$ for up to 24 hours. To address the concern of decreased ILY activity with decreasing salt concentrations, we performed salt tolerance testing in zebrafish embryos. We found that zebrafish embryos can tolerate up to $95 \mathrm{mM} \mathrm{NaCl}$ (90 mM supplemented $+5 \mathrm{mM}$ in E3 medium) without exhibiting any morphological defects or mortality. As this exceeds the $50 \mathrm{mM}$ concentration required for ILY activity, we elected to perform all experiments with E3 medium supplemented with $90 \mathrm{mM} \mathrm{NaCl}$ for a final concentration of $95 \mathrm{mM} \mathrm{NaCl}$.

ILY has previously shown to be potently hemolytic on human erythrocytes but not to nine other animal species tested including mouse, rat, chicken, rabbit, dog, cat, horse, cow and sheep (Nagamune et al., 1996). Since human CD59 receptor is more closely related to the rodent orthologue than the zebrafish, we hypothesized that ILY would not bind to zebrafish CD59 (Sun et al., 2013) and induce lysis. However, to our surprise, we found that ILY induces death at a concentration above $4 \mathrm{ng} / \mu \mathrm{l}$ in zebrafish embryos. It is likely that other components present in the ILY could be contributing to cell death in a non-specific manner but in a dose-dependent way. Performing mass spectrometry would help identify any toxic components present in ILY which might be contributing to death in ILY treated zebrafish embryos. Given the toxic nature of ILY at doses higher that $4 \mathrm{ng} / \mu \mathrm{l}$, we performed all our immersion experiments at $4 \mathrm{ng} / \mathrm{\mu l}$ concentration. Embryos transiently expressing WT hCD59 began to die 1 hour after ILY immersion leading up to $100 \%$ mortality after one day. However, embryos which 
transiently expressed the mutant form of hCD59 survived and did not exhibit any morphological defects suggesting that ILY is specific to hCD59 at lower concentrations where potential toxic components are diluted.

To further test the specificity of the hCD59/ILY system in zebrafish and to overcome some of the limitations of mRNA injection including the variation in expression levels of hCD59, we generated stable transgenic line expressing ZsGreen/hCD59 in branchiomotor neurons. Primary cells cultured from this transgenic line were rapidly (within 5 minutes) lysed by ILY treatment further validating the system. Ongoing studies aim to validate the hCD59-ILY system in intact transgenic embryos. 


\subsection{FIGURES AND LEGENDS}

Figure 6.1: Assessment of high salt and ILY-induced mortality
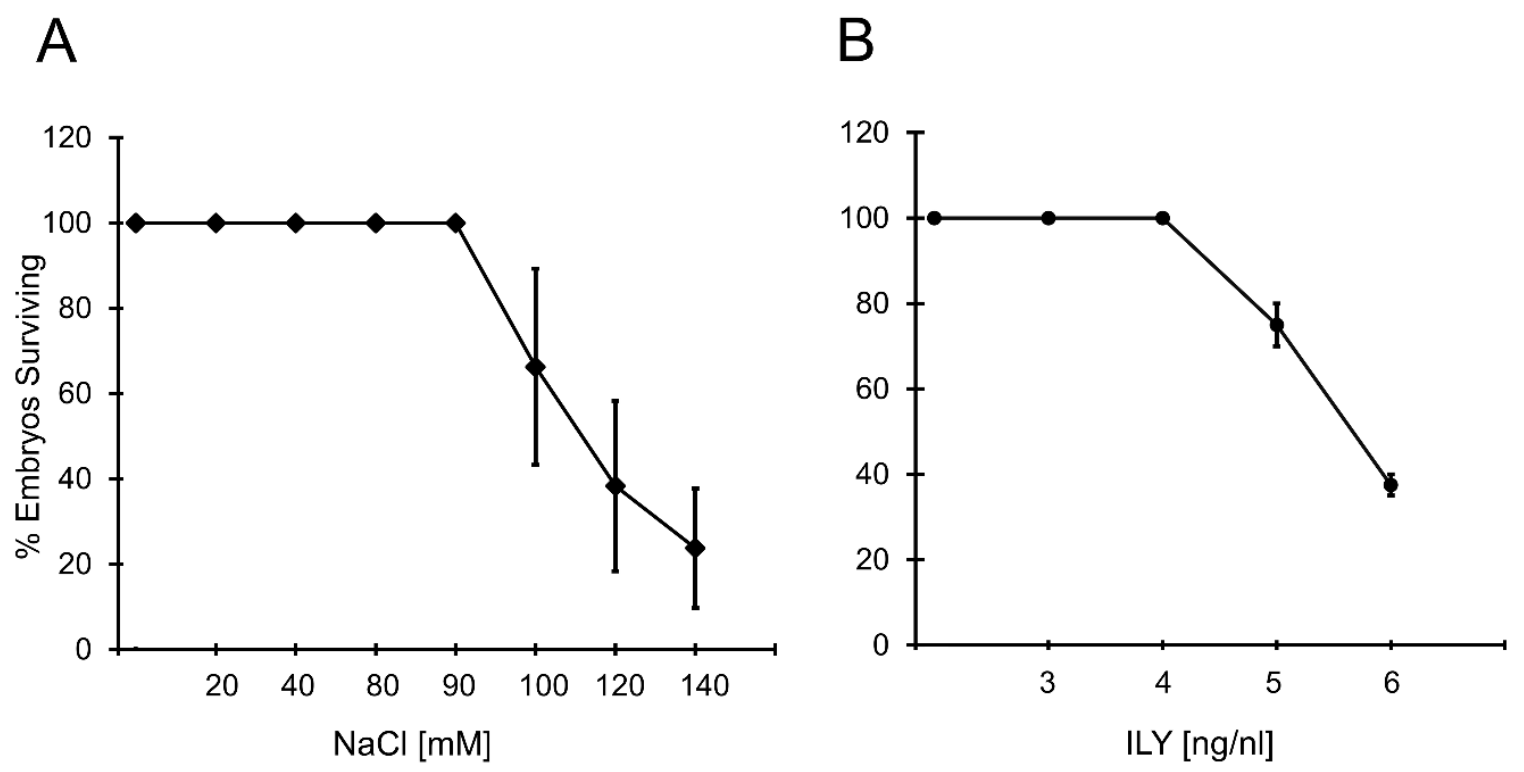
Figure 6.1 Assessment of high salt and ILY-induced mortality

(A) Wildtype (nontransgenic) embryos were treated with indicated concentrations of $\mathrm{NaCl}$ starting at $10 \mathrm{hpf}$. The number of the viable embryo was counted after overnight (14 hours) incubation at room temperature. Data are averages from two trails; $n=40$; error bars are SEM. (B) Wildtype (nontransgenic) embryos were treated with indicated concentrations of ILY at $24 \mathrm{hpf}$. The number of the viable embryo was counted after 1 hour incubation at $37^{\circ} \mathrm{C}$. Data are averages from two trails; $n=40$; error bars are SEM. 
Figure 6.2: Mortality upon exposure of mRNA injected embryos to ILY

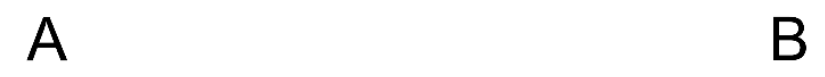

WT hCD59

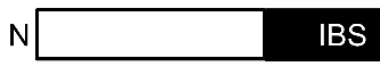

hCD59 $\triangle$ IBS
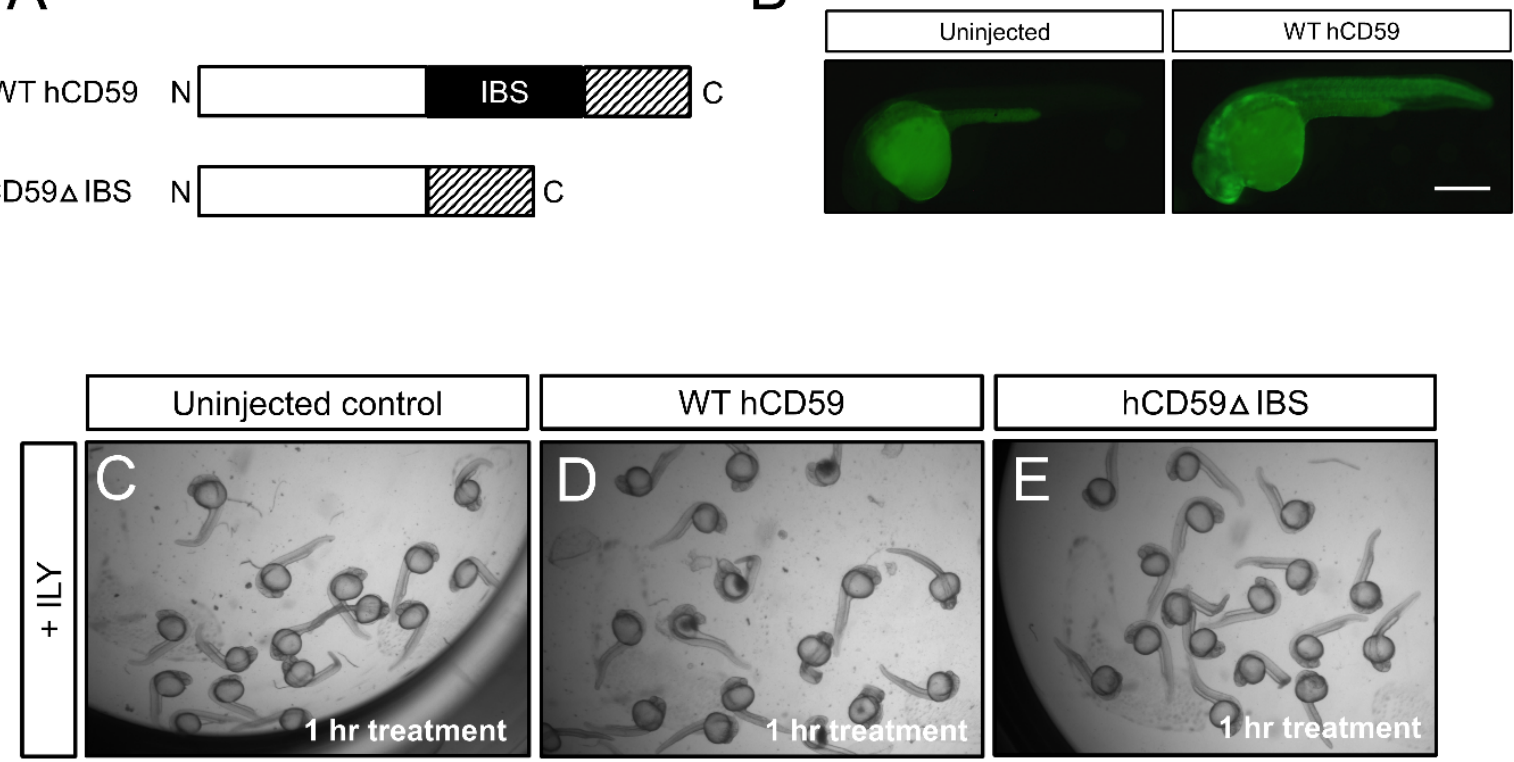

$\mathrm{F}$

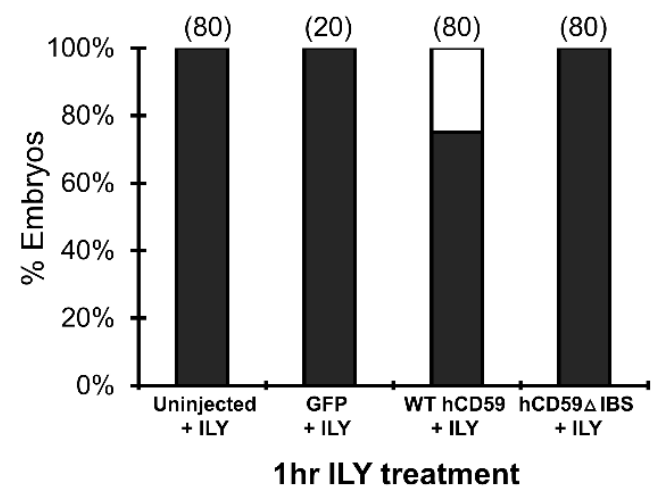

G

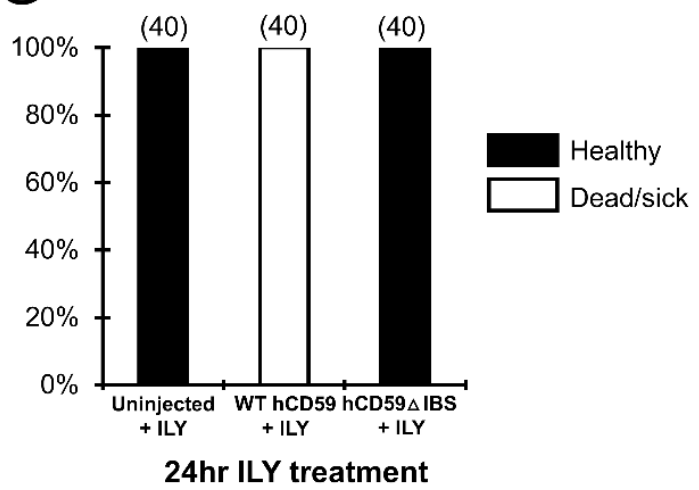


Figure 6.2: Mortality upon exposure of mRNA injected embryos to ILY

Construct used to get an expression of wildtype (WT) hCD59 and mutant hCD59 (hCD59 1 IBS) into embryos. Mutant hCD59 lacks ILY-binding domain. (B) Embryos injected with $h C D 59$ mRNA were positive for ZsGreen expression. Autofluorescence from yolk and yolk extension can be seen in both uninjected control as well as $h C D 59$ mRNA injected embryo. Lateral views of the embryo with anterior to the left. (C-E) Embryos were injected at the one-cell stage with WT $h C D 59$ mRNA, $h C D 59 \Delta / B S$ mRNA or control GFP mRNA. Uninjected embryos received no mRNA. Uninjected as well $h C D 59 \Delta / B S$ mRNA injected embryos looked morphologically normal (C, E). However, mortality was observed in WT hCD59 mRNA injected embryos post ILY treatment (D). (F, G) Quantification of mortality data after 1-hour (F) and 24 hours (G) ILY immersion. The number in parenthesis denotes the number of embryos. Data are from 2-4 experiments. Scalebar in B is $400 \mu \mathrm{m}$. 
Figure 6.3: Cell lysis upon treatment of WT hCD59-expressing embryos with ILY.

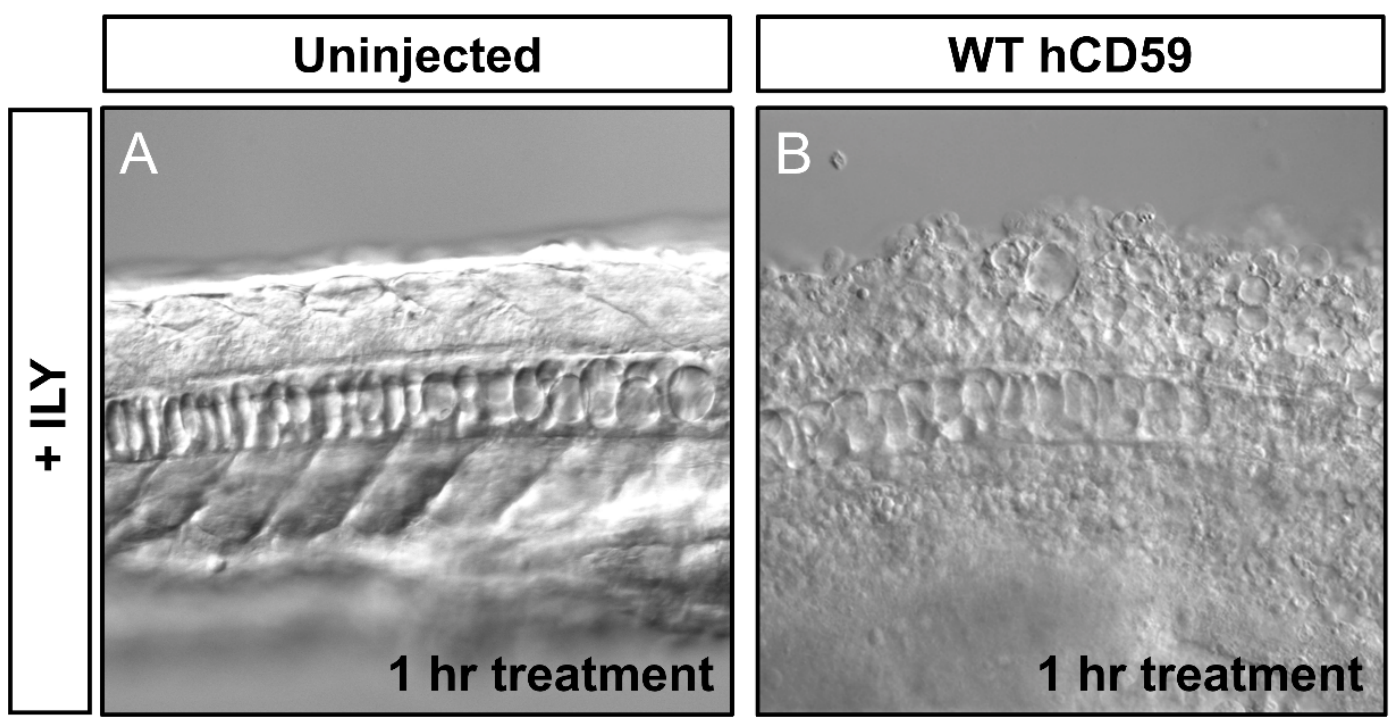


Figure 6.3: Cell lysis upon treatment of WT hCD59-expressing embryos with ILY. Lateral view of the trunk with anterior to the left. Clear somite boundaries can be seen in uninjected embryo after ILY treatment (A), however, vacuolation likely indicating lysis was observed in embryo injected with WT hCD59 mRNA after ILY treatment (B). 
Figure 6.4: Generation of transgenic line employed in this study

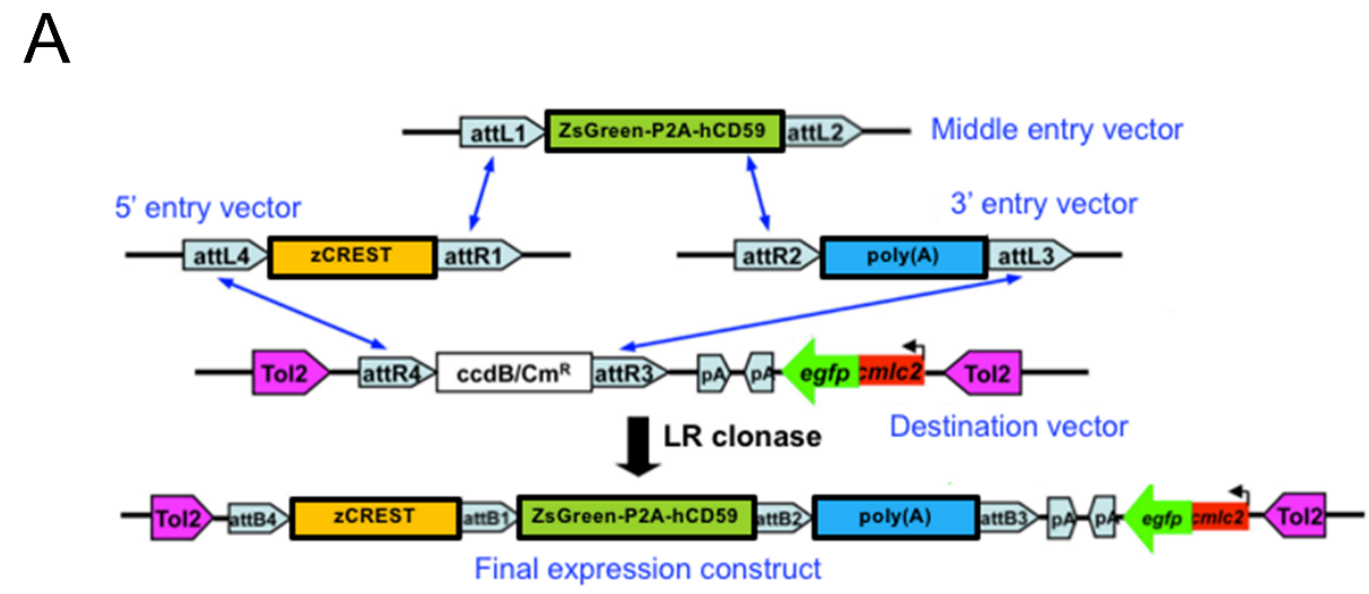

B

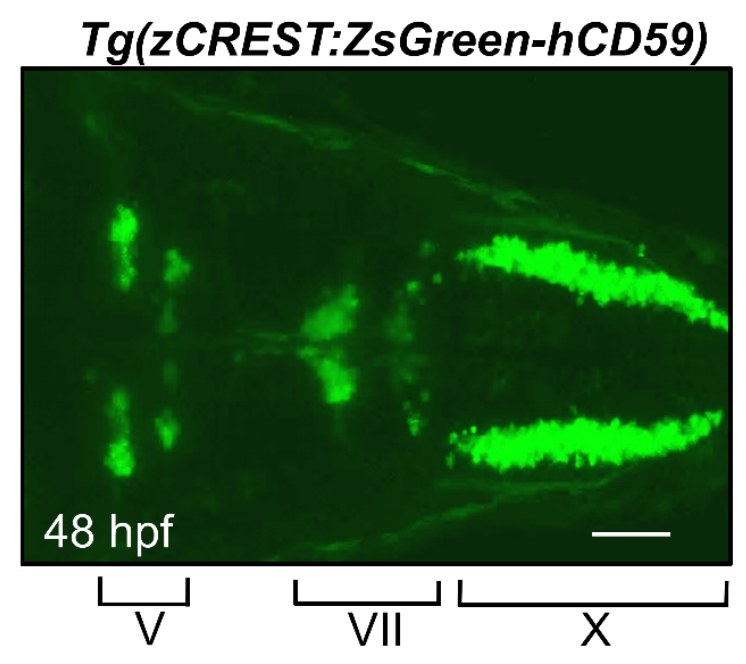


Figure 6.4: Generation of transgenic line employed in this study

(A) Schematic outlining the Gateway cloning strategy for generating a Tol2 vector to generate a stable transgenic line. The construct contains the ZsGreen-P2A-hCD59 sequence flanked by tol2 sequences. (B) Confocal z-stack of $48 \mathrm{hpf}$ $\operatorname{Tg}(z C R E S T: Z s G r e e n-h C D 59)$ embryo that was lightly fixed for 15 minutes at room temperature and mounted on PBS. ZsGreen expression can be seen in anterior and posterior clusters of trigeminal neurons (V), facial branchiomotor neurons (VII), and vagus neurons $(X)$. Dorsal view of the hindbrain with anterior to the left. 
Figure 6.5: ILY treatment in transgenic embryos

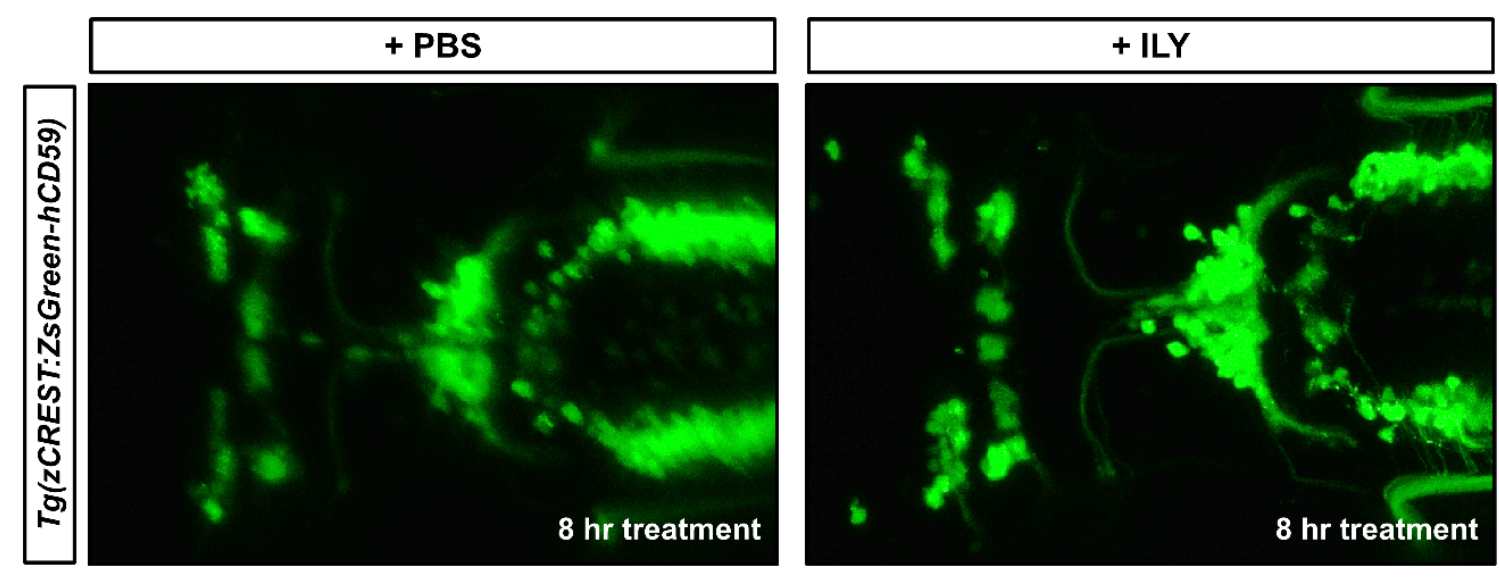


Figure 6.5: ILY treatment in transgenic embryos

Transgenic $\operatorname{Tg}(z C R E S T: Z s G r e e n-h C D 59)$ embryos expressing ZsGreen-hCD59 were treated with PBS (control) or ILY $\left(4 \mathrm{ng} / \mathrm{\mu l}\right.$ ) at $48 \mathrm{hpf}$ and incubated at $37^{\circ} \mathrm{C}$. No ablation was observed in both PBS treated (A) and ILY (B) treated embryos. Dorsal view with anterior to the left. 
Figure 6.6: ILY induces rapid ablation of hCD59-expressing cultured neurons
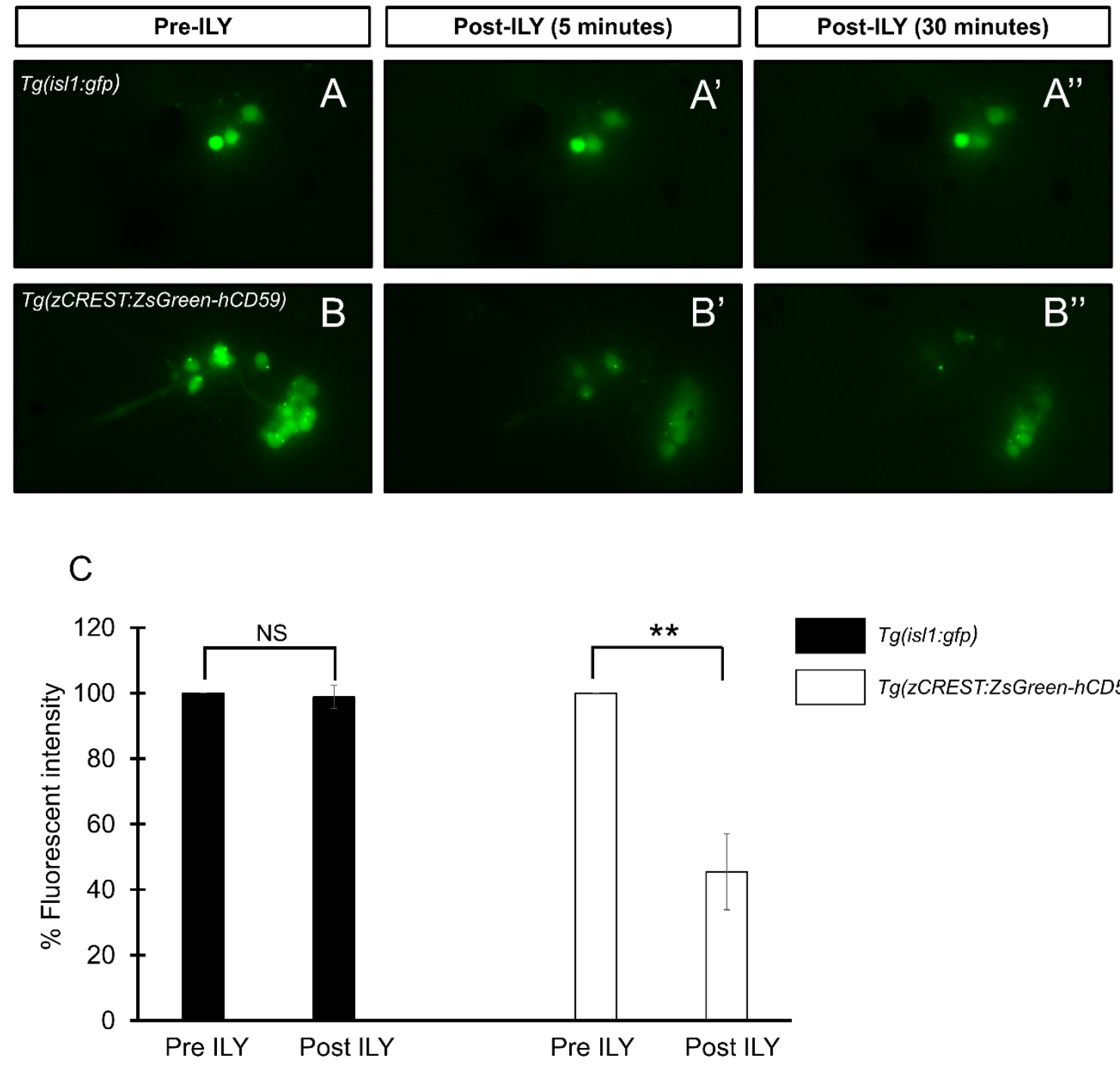
Figure 6.6: ILY induces rapid ablation of hCD59-expressing cultured neurons Primary cells were cultured from $3 \mathrm{dpf} T g$ (islet1:gfp) (A) and $\operatorname{Tg}(z C R E S T: Z s G r e e n-$ hcd59) (B) embryos and plated on poly-L-lysine coated plates. Fluorescence intensity was reduced in the majority of cells from $T g(z C R E S T: Z s G r e e n-h C D 59)$ embryos (B' and B") post ILY treatment. However, no such reduction in fluorescence intensity was seen in cells from $T g$ (islet1:gfp) embryos post ILY treatment ( $A^{\prime}$ and $\left.A^{\prime \prime}\right)$. C) Quantification of fluorescent intensity pre and post ILY treatment (30 minutes). ${ }^{* *}$ Student t-test, $p<0.001$; NS; not significant. Data are from 3 experiments. 


\section{REFERENCES}

1. Amsterdam, A., Varshney, G.K., Burgess, S.M., 2011. Retroviral-mediated Insertional Mutagenesis in Zebrafish. Methods Cell Biol 104, 59-82.

2. Andersen, E.F., Asuri, N.S., Halloran, M.C., 2011. In vivo imaging of cell behaviors and $\mathrm{F}$-actin reveals LIM-HD transcription factor regulation of peripheral versus central sensory axon development. Neural development 6, 27.

3. Ariga, J., Walker, S.L., Mumm, J.S., 2010. Multicolor time-lapse imaging of transgenic zebrafish: visualizing retinal stem cells activated by targeted neuronal cell ablation. Journal of visualized experiments : JoVE.

4. Arnaout, M.A., Goodman, S.L., Xiong, J.P., 2007. Structure and mechanics of integrinbased cell adhesion. Curr Opin Cell Biol 19, 495-507.

5. Arvanitis, D., Davy, A., 2008. Eph/ephrin signaling: networks. Genes Dev 22, 416-429.

6. Avci, H.X., Zelina, P., Thelen, K., Pollerberg, G.E., 2004. Role of cell adhesion molecule DM-GRASP in growth and orientation of retinal ganglion cell axons. Developmental biology 271, 291-305.

7. Barresi, M.J., Burton, S., Dipietrantonio, K., Amsterdam, A., Hopkins, N., Karlstrom, R.O., 2010. Essential genes for astroglial development and axon pathfinding during zebrafish embryogenesis. Developmental dynamics : an official publication of the American Association of Anatomists 239, 2603-2618.

8. Bastakis, G.G., Savvaki, M., Stamatakis, A., Vidaki, M., Karagogeos, D., 2015. Tag1 deficiency results in olfactory dysfunction through impaired migration of mitral cells. Development 142, 4318-4328.

9. Bernhardt, R.R., Chitnis, A.B., Lindamer, L., Kuwada, J.Y., 1990. Identification of spinal neurons in the embryonic and larval zebrafish. J Comp Neurol 302, 603-616.

10. Bill, B.R., Petzold, A.M., Clark, K.J., Schimmenti, L.A., Ekker, S.C., 2009. A primer for morpholino use in zebrafish. Zebrafish 6, 69-77.

11. Bilotta, J., 2000. Effects of abnormal lighting on the development of zebrafish visual behavior. Behavioural brain research 116, 81-87.

12. Bingham, S., Higashijima, S., Okamoto, H., Chandrasekhar, A., 2002. The Zebrafish trilobite gene is essential for tangential migration of branchiomotor neurons.

Developmental biology 242, 149-160.

13. Bingham, S.M., Toussaint, G., Chandrasekhar, A., 2005. Neuronal development and migration in zebrafish hindbrain explants. Journal of neuroscience methods $149,42-$ 49. 
14. Bomont, P., Cavalier, L., Blondeau, F., Ben Hamida, C., Belal, S., Tazir, M., Demir, E., Topaloglu, H., Korinthenberg, R., Tuysuz, B., Landrieu, P., Hentati, F., Koenig, M., 2000. The gene encoding gigaxonin, a new member of the cytoskeletal BTB/kelch repeat family, is mutated in giant axonal neuropathy. Nature genetics $26,370-374$.

15. Borovina, A., Superina, S., Voskas, D., Ciruna, B., 2010. Vangl2 directs the posterior tilting and asymmetric localization of motile primary cilia. Nature cell biology $12,407-$ 412.

16. Brand, M., Heisenberg, C.P., Warga, R.M., Pelegri, F., Karlstrom, R.O., Beuchle, D., Picker, A., Jiang, Y.J., Furutani-Seiki, M., van Eeden, F.J., Granato, M., Haffter, P., Hammerschmidt, M., Kane, D.A., Kelsh, R.N., Mullins, M.C., Odenthal, J., NussleinVolhard, C., 1996. Mutations affecting development of the midline and general body shape during zebrafish embryogenesis. Development 123, 129-142.

17. Brittis, P.A., Lemmon, V., Rutishauser, U., Silver, J., 1995. Unique changes of ganglion cell growth cone behavior following cell adhesion molecule perturbations: a time-lapse study of the living retina. Molecular and cellular neurosciences 6, 433-449.

18. Brose, K., Bland, K.S., Wang, K.H., Arnott, D., Henzel, W., Goodman, C.S., TessierLavigne, M., Kidd, T., 1999. Slit proteins bind Robo receptors and have an evolutionarily conserved role in repulsive axon guidance. Cell 96, 795-806.

19. Brummendorf, T., Rathjen, F.G., 1996. Structure/function relationships of axonassociated adhesion receptors of the immunoglobulin superfamily. Current opinion in neurobiology $6,584-593$.

20. Bruno, C., Bertini, E., Federico, A., Tonoli, E., Lispi, M.L., Cassandrini, D., Pedemonte, M., Santorelli, F.M., Filocamo, M., Dotti, M.T., Schenone, A., Malandrini, A., Minetti, C., 2004. Clinical and molecular findings in patients with giant axonal neuropathy (GAN). Neurology 62, 13-16.

21. Buch, T., Heppner, F.L., Tertilt, C., Heinen, T.J., Kremer, M., Wunderlich, F.T., Jung, S., Waisman, A., 2005. A Cre-inducible diphtheria toxin receptor mediates cell lineage ablation after toxin administration. Nat Methods 2, 419-426.

22. Buchstaller, A., Kunz, S., Berger, P., Kunz, B., Ziegler, U., Rader, C., Sonderegger, P., 1996. Cell adhesion molecules NgCAM and axonin-1 form heterodimers in the neuronal membrane and cooperate in neurite outgrowth promotion. The Journal of cell biology $135,1593-1607$.

23. Caddy, J., Wilanowski, T., Darido, C., Dworkin, S., Ting, S.B., Zhao, Q., Rank, G., Auden, A., Srivastava, S., Papenfuss, T.A., Murdoch, J.N., Humbert, P.O., Parekh, V., Boulos, N., Weber, T., Zuo, J., Cunningham, J.M., Jane, S.M., 2010. Epidermal wound repair is regulated by the planar cell polarity signaling pathway. Developmental cell 19 , 138-147. 
24. Campbell, K., Gotz, M., 2002. Radial glia: multi-purpose cells for vertebrate brain development. Trends in neurosciences 25, 235-238.

25. Cantrell, V.A., Jessen, J.R., 2010. The planar cell polarity protein Van Gogh-Like 2 regulates tumor cell migration and matrix metalloproteinase-dependent invasion. Cancer letters 287, 54-61.

26. Carmean, V., Ribera, A.B., 2010. Genetic Analysis of the Touch Response in Zebrafish (). Int J Comp Psychol 23, 91.

27. Carreira-Barbosa, F., Concha, M.L., Takeuchi, M., Ueno, N., Wilson, S.W., Tada, M., 2003. Prickle 1 regulates cell movements during gastrulation and neuronal migration in zebrafish. Development 130, 4037-4046.

28. Chandrasekhar, A., 2004. Turning heads: development of vertebrate branchiomotor neurons. Developmental dynamics : an official publication of the American Association of Anatomists 229, 143-161.

29. Chandrasekhar, A., Moens, C.B., Warren, J.T., Jr., Kimmel, C.B., Kuwada, J.Y., 1997. Development of branchiomotor neurons in zebrafish. Development 124, 2633-2644.

30. Chandrasekhar, A., Schauerte, H.E., Haffter, P., Kuwada, J.Y., 1999. The zebrafish detour gene is essential for cranial but not spinal motor neuron induction. Development $126,2727-2737$.

31. Chang, Y.F., Imam, J.S., Wilkinson, M.F., 2007. The nonsense-mediated decay RNA surveillance pathway. Annu Rev Biochem 76, 51-74.

32. Chao, D.L., Ma, L., Shen, K., 2009. Transient cell-cell interactions in neural circuit formation. Nature reviews. Neuroscience 10, 262-271.

33. Chatzopoulou, E., Miguez, A., Savvaki, M., Levasseur, G., Muzerelle, A., Muriel, M.P., Goureau, O., Watanabe, K., Goutebroze, L., Gaspar, P., Zalc, B., Karagogeos, D., Thomas, J.L., 2008. Structural requirement of TAG-1 for retinal ganglion cell axons and myelin in the mouse optic nerve. The Journal of neuroscience : the official journal of the Society for Neuroscience 28, 7624-7636.

34. Chen, C.F., Chu, C.Y., Chen, T.H., Lee, S.J., Shen, C.N., Hsiao, C.D., 2011. Establishment of a transgenic zebrafish line for superficial skin ablation and functional validation of apoptosis modulators in vivo. PLoS One 6, e20654.

35. Chen, J.H., Wu, W., Li, H.S., Fagaly, T., Zhou, L., Wu, J.Y., Rao, Y., 2000. Embryonic expression and extracellular secretion of Xenopus slit. Neuroscience 96, 231-236.

36. Chin-Sang, I.D., George, S.E., Ding, M., Moseley, S.L., Lynch, A.S., Chisholm, A.D., 1999. The ephrin VAB-2/EFN-1 functions in neuronal signaling to regulate epidermal morphogenesis in C. elegans. Cell 99, 781-790. 
37. Chitnis, A.B., Kuwada, J.Y., 1990. Axonogenesis in the brain of zebrafish embryos. The Journal of neuroscience : the official journal of the Society for Neuroscience 10 , 1892-1905.

38. Ciruna, B., Jenny, A., Lee, D., Mlodzik, M., Schier, A.F., 2006. Planar cell polarity signalling couples cell division and morphogenesis during neurulation. Nature 439, 220-224.

39. Cloutier, J.F., Sahay, A., Chang, E.C., Tessier-Lavigne, M., Dulac, C., Kolodkin, A.L., Ginty, D.D., 2004. Differential requirements for semaphorin 3F and Slit-1 in axonal targeting, fasciculation, and segregation of olfactory sensory neuron projections. The Journal of neuroscience : the official journal of the Society for Neuroscience 24, 90879096.

40. Cooper, K.L., Armstrong, J., Moens, C.B., 2005. Zebrafish foggy/spt 5 is required for migration of facial branchiomotor neurons but not for their survival. Developmental dynamics : an official publication of the American Association of Anatomists 234, 651658.

41. Cubedo, N., Cerdan, E., Sapede, D., Rossel, M., 2009. CXCR4 and CXCR7 cooperate during tangential migration of facial motoneurons. Molecular and cellular neurosciences $40,474-484$.

42. Culotti, J.G., Merz, D.C., 1998. DCC and netrins. Curr Opin Cell Biol 10, 609-613.

43. Cunningham, B.A., Hemperly, J.J., Murray, B.A., Prediger, E.A., Brackenbury, R., Edelman, G.M., 1987. Neural cell adhesion molecule: structure, immunoglobulin-like domains, cell surface modulation, and alternative RNA splicing. Science 236, 799-806.

44. Curado, S., Anderson, R.M., Jungblut, B., Mumm, J., Schroeter, E., Stainier, D.Y., 2007. Conditional targeted cell ablation in zebrafish: a new tool for regeneration studies. Developmental dynamics : an official publication of the American Association of Anatomists 236, 1025-1035.

45. Curado, S., Stainier, D.Y., Anderson, R.M., 2008. Nitroreductase-mediated cell/tissue ablation in zebrafish: a spatially and temporally controlled ablation method with applications in developmental and regeneration studies. Nature protocols 3, 948-954.

46. Darken, R.S., Scola, A.M., Rakeman, A.S., Das, G., Mlodzik, M., Wilson, P.A., 2002. The planar polarity gene strabismus regulates convergent extension movements in Xenopus. The EMBO journal 21, 976-985.

47. Davey, C.F., Mathewson, A.W., Moens, C.B., 2016. PCP Signaling between Migrating Neurons and their Planar-Polarized Neuroepithelial Environment Controls Filopodial Dynamics and Directional Migration. PLoS Genet 12, e1005934.

48. Davies, A., Simmons, D.L., Hale, G., Harrison, R.A., Tighe, H., Lachmann, P.J., Waldmann, H., 1989. CD59, an LY-6-like protein expressed in human lymphoid cells, 
regulates the action of the complement membrane attack complex on homologous cells. J Exp Med 170, 637-654.

49. de la Rosa, E.J., Kayyem, J.F., Roman, J.M., Stierhof, Y.D., Dreyer, W.J., Schwarz, U., 1990. Topologically restricted appearance in the developing chick retinotectal system of Bravo, a neural surface protein: experimental modulation by environmental cues. The Journal of cell biology 111, 3087-3096.

50. Deiner, M.S., Kennedy, T.E., Fazeli, A., Serafini, T., Tessier-Lavigne, M., Sretavan, D.W., 1997. Netrin-1 and DCC mediate axon guidance locally at the optic disc: loss of function leads to optic nerve hypoplasia. Neuron 19, 575-589.

51. Dodd, J., Morton, S.B., Karagogeos, D., Yamamoto, M., Jessell, T.M., 1988. Spatial regulation of axonal glycoprotein expression on subsets of embryonic spinal neurons. Neuron 1, 105-116.

52. Dornfest, B.S., Bush, M.E., Lapin, D.M., Adu, S., Fulop, A., Naughton, B.A., 1990. Phenylhydrazine is a mitogen and activator of lymphoid cells. Annals of clinical and laboratory science $20,353-370$.

53. Eisen, J.S., Smith, J.C., 2008. Controlling morpholino experiments: don't stop making antisense. Development 135, 1735-1743.

54. Elsen, G.E., Choi, L.Y., Prince, V.E., Ho, R.K., 2009. The autism susceptibility gene met regulates zebrafish cerebellar development and facial motor neuron migration. Developmental biology 335, 78-92.

55. Engle, E.C., 2010. Human genetic disorders of axon guidance. Cold Spring Harb Perspect Biol 2, a001784.

56. Essner, J.J., Branford, W.W., Zhang, J., Yost, H.J., 2000. Mesendoderm and left-right brain, heart and gut development are differentially regulated by pitx2 isoforms.

Development 127, 1081-1093.

57. Falk, J., Bechara, A., Fiore, R., Nawabi, H., Zhou, H., Hoyo-Becerra, C., Bozon, M., Rougon, G., Grumet, M., Puschel, A.W., Sanes, J.R., Castellani, V., 2005. Dual functional activity of semaphorin $3 \mathrm{~B}$ is required for positioning the anterior commissure. Neuron 48, 63-75.

58. Falk, J., Bonnon, C., Girault, J.A., Faivre-Sarrailh, C., 2002. F3/contactin, a neuronal cell adhesion molecule implicated in axogenesis and myelination. Biology of the cell 94, 327-334.

59. Feng, D., Dai, S., Liu, F., Ohtake, Y., Zhou, Z., Wang, H., Zhang, Y., Kearns, A., Peng, X., Zhu, F., Hayat, U., Li, M., He, Y., Xu, M., Zhao, C., Cheng, M., Zhang, L., Wang, H., Yang, X., Ju, C., Bryda, E.C., Gordon, J., Khalili, K., Hu, W., Li, S., Qin, X., Gao, B., 2016. Cre-inducible human CD59 mediates rapid cell ablation after intermedilysin administration. The Journal of clinical investigation 126, 2321-2333. 
60. Fitzli, D., Stoeckli, E.T., Kunz, S., Siribour, K., Rader, C., Kunz, B., Kozlov, S.V., Buchstaller, A., Lane, R.P., Suter, D.M., Dreyer, W.J., Sonderegger, P., 2000. A direct interaction of axonin-1 with NgCAM-related cell adhesion molecule (NrCAM) results in guidance, but not growth of commissural axons. The Journal of cell biology 149, 951968.

61. Francks, C., Maegawa, S., Lauren, J., Abrahams, B.S., Velayos-Baeza, A., Medland, S.E., Colella, S., Groszer, M., McAuley, E.Z., Caffrey, T.M., Timmusk, T., Pruunsild, P., Koppel, I., Lind, P.A., Matsumoto-Itaba, N., Nicod, J., Xiong, L., Joober, R., Enard, W., Krinsky, B., Nanba, E., Richardson, A.J., Riley, B.P., Martin, N.G., Strittmatter, S.M., Moller, H.J., Rujescu, D., St Clair, D., Muglia, P., Roos, J.L., Fisher, S.E., WadeMartins, R., Rouleau, G.A., Stein, J.F., Karayiorgou, M., Geschwind, D.H., Ragoussis, J., Kendler, K.S., Airaksinen, M.S., Oshimura, M., DeLisi, L.E., Monaco, A.P., 2007. LRRTM1 on chromosome 2p12 is a maternally suppressed gene that is associated paternally with handedness and schizophrenia. Molecular psychiatry 12, 1129-1139, 1057.

62. Furley, A.J., Morton, S.B., Manalo, D., Karagogeos, D., Dodd, J., Jessell, T.M., 1990. The axonal glycoprotein TAG-1 is an immunoglobulin superfamily member with neurite outgrowth-promoting activity. Cell 61, 157-170.

63. Gahtan, E., Baier, H., 2004. Of lasers, mutants, and see-through brains: functional neuroanatomy in zebrafish. Journal of neurobiology 59, 147-161.

64. Gahtan, E., Tanger, P., Baier, H., 2005. Visual prey capture in larval zebrafish is controlled by identified reticulospinal neurons downstream of the tectum. The Journal of neuroscience : the official journal of the Society for Neuroscience 25, 9294-9303.

65. Garel, S., Garcia-Dominguez, M., Charnay, P., 2000. Control of the migratory pathway of facial branchiomotor neurones. Development 127, 5297-5307.

66. Gennarini, G., Bizzoca, A., Picocci, S., Puzzo, D., Corsi, P., Furley, A.J., 2016. The role of Gpi-anchored axonal glycoproteins in neural development and neurological disorders. Molecular and cellular neurosciences.

67. George, S.E., Simokat, K., Hardin, J., Chisholm, A.D., 1998. The VAB-1 Eph receptor tyrosine kinase functions in neural and epithelial morphogenesis in C. elegans. Cell 92, 633-643.

68. Giddings, K.S., Johnson, A.E., Tweten, R.K., 2003. Redefining cholesterol's role in the mechanism of the cholesterol-dependent cytolysins. Proceedings of the National Academy of Sciences of the United States of America 100, 11315-11320.

69. Giddings, K.S., Zhao, J., Sims, P.J., Tweten, R.K., 2004. Human CD59 is a receptor for the cholesterol-dependent cytolysin intermedilysin. Nature structural \& molecular biology 11, 1173-1178. 
70. Gilland, E., Baker, R., 2005. Evolutionary patterns of cranial nerve efferent nuclei in vertebrates. Brain, behavior and evolution 66, 234-254.

71. Glasco, D.M., Sittaramane, V., Bryant, W., Fritzsch, B., Sawant, A., Paudyal, A., Stewart, M., Andre, P., Cadete Vilhais-Neto, G., Yang, Y., Song, M.R., Murdoch, J.N., Chandrasekhar, A., 2012. The mouse Wnt/PCP protein Vangl2 is necessary for migration of facial branchiomotor neurons, and functions independently of Dishevelled. Developmental biology 369, 211-222.

72. Golling, G., Amsterdam, A., Sun, Z., Antonelli, M., Maldonado, E., Chen, W., Burgess, S., Haldi, M., Artzt, K., Farrington, S., Lin, S.Y., Nissen, R.M., Hopkins, N., 2002. Insertional mutagenesis in zebrafish rapidly identifies genes essential for early vertebrate development. Nature genetics 31, 135-140.

73. Granato, M., van Eeden, F.J., Schach, U., Trowe, T., Brand, M., Furutani-Seiki, M., Haffter, P., Hammerschmidt, M., Heisenberg, C.P., Jiang, Y.J., Kane, D.A., Kelsh, R.N., Mullins, M.C., Odenthal, J., Nusslein-Volhard, C., 1996. Genes controlling and mediating locomotion behavior of the zebrafish embryo and larva. Development 123, 399-413.

74. Grant, P.K., Moens, C.B., 2010. The neuroepithelial basement membrane serves as a boundary and a substrate for neuron migration in the zebrafish hindbrain. Neural development $5,9$.

75. Gray, R.S., Roszko, I., Solnica-Krezel, L., 2011. Planar cell polarity: coordinating morphogenetic cell behaviors with embryonic polarity. Developmental cell 21, 120-133.

76. Grohmann, M., Paulmann, N., Fleischhauer, S., Vowinckel, J., Priller, J., Walther, D.J., 2009. A mammalianized synthetic nitroreductase gene for high-level expression. BMC cancer 9, 301.

77. Hamlin, J.A., Fang, H., Schwob, J.E., 2004. Differential expression of the mammalian homologue of fasciclin II during olfactory development in vivo and in vitro. J Comp Neurol 474, 438-452.

78. Hammerschmidt, M., Pelegri, F., Mullins, M.C., Kane, D.A., Brand, M., van Eeden, F.J., Furutani-Seiki, M., Granato, M., Haffter, P., Heisenberg, C.P., Jiang, Y.J., Kelsh, R.N., Odenthal, J., Warga, R.M., Nusslein-Volhard, C., 1996. Mutations affecting morphogenesis during gastrulation and tail formation in the zebrafish, Danio rerio. Development 123, 143-151.

79. Hao, J.C., Yu, T.W., Fujisawa, K., Culotti, J.G., Gengyo-Ando, K., Mitani, S., Moulder, G., Barstead, R., Tessier-Lavigne, M., Bargmann, C.I., 2001. C. elegans slit acts in midline, dorsal-ventral, and anterior-posterior guidance via the SAX-3/Robo receptor. Neuron 32, 25-38.

80. Hatten, M.E., 1999. Central nervous system neuronal migration. Annual review of neuroscience 22, 511-539. 
81. Hatten, M.E., 2002. New directions in neuronal migration. Science 297, 1660-1663.

82. Hedgecock, E.M., Culotti, J.G., Hall, D.H., 1990. The unc-5, unc-6, and unc-40 genes guide circumferential migrations of pioneer axons and mesodermal cells on the epidermis in C. elegans. Neuron 4, 61-85.

83. Higashijima, S., Hotta, Y., Okamoto, H., 2000. Visualization of cranial motor neurons in live transgenic zebrafish expressing green fluorescent protein under the control of the islet-1 promoter/enhancer. The Journal of neuroscience : the official journal of the Society for Neuroscience 20, 206-218.

84. Hjorth, J., Key, B., 2002. Development of axon pathways in the zebrafish central nervous system. The International journal of developmental biology 46, 609-619.

85. Hoffman, E.J., Turner, K.J., Fernandez, J.M., Cifuentes, D., Ghosh, M., ljaz, S., Jain, R.A., Kubo, F., Bill, B.R., Baier, H., Granato, M., Barresi, M.J., Wilson, S.W., Rihel, J., State, M.W., Giraldez, A.J., 2016. Estrogens Suppress a Behavioral Phenotype in Zebrafish Mutants of the Autism Risk Gene, CNTNAP2. Neuron 89, 725-733.

86. Holmes, G., Niswander, L., 2001. Expression of slit-2 and slit-3 during chick development. Developmental dynamics : an official publication of the American Association of Anatomists 222, 301-307.

87. Hong, E., Brewster, R., 2006. N-cadherin is required for the polarized cell behaviors that drive neurulation in the zebrafish. Development 133, 3895-3905.

88. Hong, K., Hinck, L., Nishiyama, M., Poo, M.M., Tessier-Lavigne, M., Stein, E., 1999. A ligand-gated association between cytoplasmic domains of UNC5 and DCC family receptors converts netrin-induced growth cone attraction to repulsion. Cell 97, 927941.

89. Horwitz, A.R., Parsons, J.T., 1999. Cell migration--movin' on. Science 286, 1102-1103.

90. Hu, W., Ferris, S.P., Tweten, R.K., Wu, G., Radaeva, S., Gao, B., Bronson, R.T., Halperin, J.A., Qin, X., 2008. Rapid conditional targeted ablation of cells expressing human CD59 in transgenic mice by intermedilysin. Nature medicine 14, 98-103.

91. Huber, A.B., Kania, A., Tran, T.S., Gu, C., De Marco Garcia, N., Lieberam, I., Johnson, D., Jessell, T.M., Ginty, D.D., Kolodkin, A.L., 2005. Distinct roles for secreted semaphorin signaling in spinal motor axon guidance. Neuron 48, 949-964.

92. Huber, A.B., Kolodkin, A.L., Ginty, D.D., Cloutier, J.F., 2003. Signaling at the growth cone: ligand-receptor complexes and the control of axon growth and guidance. Annual review of neuroscience $26,509-563$.

93. Hughes, T.R., Ross, K.S., Cowan, G.J., Sivasankar, B., Harris, C.L., Mitchell, T.J., Morgan, B.P., 2009. Identification of the high affinity binding site in the Streptococcus 
intermedius toxin intermedilysin for its membrane receptor, the human complement regulator CD59. Molecular immunology 46, 1561-1567.

94. Hummel, T., Schimmelpfeng, K., Klambt, C., 1999. Commissure formation in the embryonic CNS of Drosophila. Developmental biology 209, 381-398.

95. Hutson, L.D., Chien, C.B., 2002. Pathfinding and error correction by retinal axons: the role of astray/robo2. Neuron 33, 205-217.

96. Hwang, W.Y., Fu, Y., Reyon, D., Maeder, M.L., Tsai, S.Q., Sander, J.D., Peterson, R.T., Yeh, J.R., Joung, J.K., 2013. Efficient genome editing in zebrafish using a CRISPR-Cas system. Nature biotechnology 31, 227-229.

97. Hynes, R.O., 2002. Integrins: bidirectional, allosteric signaling machines. Cell 110, 673-687.

98. Ingold, E., Vom Berg-Maurer, C.M., Burckhardt, C.J., Lehnherr, A., Rieder, P., Keller, P.J., Stelzer, E.H., Greber, U.F., Neuhauss, S.C., Gesemann, M., 2015. Proper migration and axon outgrowth of zebrafish cranial motoneuron subpopulations require the cell adhesion molecule MDGA2A. Biol Open 4, 146-154.

99. Inoue, A., Takahashi, M., Hatta, K., Hotta, Y., Okamoto, H., 1994. Developmental regulation of islet-1 mRNA expression during neuronal differentiation in embryonic zebrafish. Developmental dynamics : an official publication of the American Association of Anatomists 199, 1-11.

100. Jao, L.E., Wente, S.R., Chen, W., 2013. Efficient multiplex biallelic zebrafish genome editing using a CRISPR nuclease system. Proceedings of the National Academy of Sciences of the United States of America 110, 13904-13909.

101. Jessell, T.M., 2000. Neuronal specification in the spinal cord: inductive signals and transcriptional codes. Nature reviews. Genetics 1, 20-29.

102. Jessen, J.R., Topczewski, J., Bingham, S., Sepich, D.S., Marlow, F., Chandrasekhar, A., Solnica-Krezel, L., 2002. Zebrafish trilobite identifies new roles for Strabismus in gastrulation and neuronal movements. Nature cell biology 4, 610-615.

103. Kaprielian, Z., Runko, E., Imondi, R., 2001. Axon guidance at the midline choice point. Developmental dynamics : an official publication of the American Association of Anatomists 221, 154-181.

104. Karlstrom, R.O., Trowe, T., Klostermann, S., Baier, H., Brand, M., Crawford, A.D., Grunewald, B., Haffter, P., Hoffmann, H., Meyer, S.U., Muller, B.K., Richter, S., van Eeden, F.J., Nusslein-Volhard, C., Bonhoeffer, F., 1996. Zebrafish mutations affecting retinotectal axon pathfinding. Development 123, 427-438.

105. Katoh, M., 2005. WNT/PCP signaling pathway and human cancer (review). Oncology reports $14,1583-1588$. 
106. Kidd, T., Brose, K., Mitchell, K.J., Fetter, R.D., Tessier-Lavigne, M., Goodman, C.S., Tear, G., 1998. Roundabout controls axon crossing of the CNS midline and defines a novel subfamily of evolutionarily conserved guidance receptors. Cell 92, 205-215.

107. Kikuta, H., Kawakami, K., 2009. Transient and stable transgenesis using tol2 transposon vectors. Methods Mol Biol 546, 69-84.

108. Kimmel, C.B., Ballard, W.W., Kimmel, S.R., Ullmann, B., Schilling, T.F., 1995. Stages of embryonic development of the zebrafish. Developmental dynamics : an official publication of the American Association of Anatomists 203, 253-310.

109. Kimmel, C.B., Powell, S.L., Metcalfe, W.K., 1982. Brain neurons which project to the spinal cord in young larvae of the zebrafish. J Comp Neurol 205, 112-127.

110. Kitsukawa, T., Shimizu, M., Sanbo, M., Hirata, T., Taniguchi, M., Bekku, Y., Yagi, T., Fujisawa, H., 1997. Neuropilin-semaphorin III/D-mediated chemorepulsive signals play a crucial role in peripheral nerve projection in mice. Neuron 19, 995-1005.

111. Klinken, S.P., Holmes, K.L., Fredrickson, T.N., Erner, S.M., Morse, H.C., 3rd, 1987. Phenylhydrazine stimulates lymphopoiesis and accelerates Abelson murine leukemia virus-induced pre-B cell lymphomas. Journal of immunology 139, 3091-3098.

112. Knox, R.J., Friedlos, F., Jarman, M., Roberts, J.J., 1988. A new cytotoxic, DNA interstrand crosslinking agent, 5-(aziridin-1-yl)-4-hydroxylamino-2-nitrobenzamide, is formed from 5-(aziridin-1-yl)-2,4-dinitrobenzamide (CB 1954) by a nitroreductase enzyme in Walker carcinoma cells. Biochemical pharmacology 37, 4661-4669.

113. Kobe, B., Kajava, A.V., 2001. The leucine-rich repeat as a protein recognition motif. Current opinion in structural biology $11,725-732$.

114. Kok, F.O., Shin, M., Ni, C.W., Gupta, A., Grosse, A.S., van Impel, A., Kirchmaier, B.C., Peterson-Maduro, J., Kourkoulis, G., Male, I., DeSantis, D.F., Sheppard-Tindell, S., Ebarasi, L., Betsholtz, C., Schulte-Merker, S., Wolfe, S.A., Lawson, N.D., 2015. Reverse genetic screening reveals poor correlation between morpholino-induced and mutant phenotypes in zebrafish. Developmental cell 32, 97-108.

115. Koshkaryev, A., Barshtein, G., Nyska, A., Ezov, N., Levin-Harrus, T., Shabat, S., Nyska, M., Redlich, M., Tsipis, F., Yedgar, S., 2003. 2-Butoxyethanol enhances the adherence of red blood cells. Arch Toxicol 77, 465-469.

116. Kuhn, T.B., Stoeckli, E.T., Condrau, M.A., Rathjen, F.G., Sonderegger, P., 1991. Neurite outgrowth on immobilized axonin-1 is mediated by a heterophilic interaction with L1(G4). The Journal of cell biology 115, 1113-1126.

117. Kunz, B., Lierheimer, R., Rader, C., Spirig, M., Ziegler, U., Sonderegger, P., 2002. Axonin-1/TAG-1 mediates cell-cell adhesion by a cis-assisted trans-interaction. The Journal of biological chemistry $277,4551-4557$. 
118. Kunz, S., Spirig, M., Ginsburg, C., Buchstaller, A., Berger, P., Lanz, R., Rader, C., Vogt, L., Kunz, B., Sonderegger, P., 1998. Neurite fasciculation mediated by complexes of axonin-1 and $\mathrm{Ng}$ cell adhesion molecule. The Journal of cell biology 143, 1673-1690.

119. Kurita, R., Sagara, H., Aoki, Y., Link, B.A., Arai, K., Watanabe, S., 2003. Suppression of lens growth by alphaA-crystallin promoter-driven expression of diphtheria toxin results in disruption of retinal cell organization in zebrafish. Developmental biology 255, 113-127.

120. Kwan, K.M., Fujimoto, E., Grabher, C., Mangum, B.D., Hardy, M.E., Campbell, D.S., Parant, J.M., Yost, H.J., Kanki, J.P., Chien, C.B., 2007. The Tol2kit: a multisite gateway-based construction kit for Tol2 transposon transgenesis constructs. Developmental dynamics : an official publication of the American Association of Anatomists 236, 3088-3099.

121. Lahl, K., Loddenkemper, C., Drouin, C., Freyer, J., Arnason, J., Eberl, G., Hamann, A., Wagner, H., Huehn, J., Sparwasser, T., 2007. Selective depletion of Foxp3+ regulatory T cells induces a scurfy-like disease. J Exp Med 204, 57-63.

122. Lang, D.M., Warren, J.T., Jr., Klisa, C., Stuermer, C.A., 2001. Topographic restriction of TAG-1 expression in the developing retinotectal pathway and target dependent reexpression during axon regeneration. Molecular and cellular neurosciences 17, 398414.

123. Law, C.O., Kirby, R.J., Aghamohammadzadeh, S., Furley, A.J., 2008. The neural adhesion molecule TAG-1 modulates responses of sensory axons to diffusible guidance signals. Development 135, 2361-2371.

124. Lebedeva, S., de Jesus Domingues, A.M., Butter, F., Ketting, R.F., 2017. Characterization of genetic loss-of-function of Fus in zebrafish. RNA Biol 14, 29-35.

125. Lee, B., Lam, D.T., Baek, K., Yoon, J., Jeong, Y., 2015. Conditional cell ablation via diphtheria toxin reveals distinct requirements for the basal plate in the regional identity of diencephalic subpopulations. Genesis (New York, N.Y. : 2000) 53, 356-365.

126. Lele, Z., Folchert, A., Concha, M., Rauch, G.J., Geisler, R., Rosa, F., Wilson, S.W., Hammerschmidt, M., Bally-Cuif, L., 2002. parachute/n-cadherin is required for morphogenesis and maintained integrity of the zebrafish neural tube. Development $129,3281-3294$.

127. Lewandoski, M., 2001. Conditional control of gene expression in the mouse. Nature reviews. Genetics 2, 743-755.

128. Li, X., Roszko, I., Sepich, D.S., Ni, M., Hamm, H.E., Marlow, F.L., Solnica-Krezel, L., 2013. Gpr125 modulates Dishevelled distribution and planar cell polarity signaling. Development 140, 3028-3039. 
129. Linhoff, M.W., Lauren, J., Cassidy, R.M., Dobie, F.A., Takahashi, H., Nygaard, H.B., Airaksinen, M.S., Strittmatter, S.M., Craig, A.M., 2009. An unbiased expression screen for synaptogenic proteins identifies the LRRTM protein family as synaptic organizers. Neuron 61, 734-749.

130. Lisabeth, E.M., Falivelli, G., Pasquale, E.B., 2013. Eph receptor signaling and ephrins. Cold Spring Harb Perspect Biol 5.

131. Liu, Y., Halloran, M.C., 2005. Central and peripheral axon branches from one neuron are guided differentially by Semaphorin3D and transient axonal glycoprotein-1. The Journal of neuroscience : the official journal of the Society for Neuroscience 25, 10556-10563.

132. Logan, C.Y., Nusse, R., 2004. The Wnt signaling pathway in development and disease. Annual review of cell and developmental biology 20, 781-810.

133. Lois, C., Garcia-Verdugo, J.M., Alvarez-Buylla, A., 1996. Chain migration of neuronal precursors. Science 271, 978-981.

134. Long, H., Sabatier, C., Ma, L., Plump, A., Yuan, W., Ornitz, D.M., Tamada, A., Murakami, F., Goodman, C.S., Tessier-Lavigne, M., 2004. Conserved roles for Slit and Robo proteins in midline commissural axon guidance. Neuron 42, 213-223.

135. Love, C.E., Prince, V.E., 2015. Rest represses maturation within migrating facial branchiomotor neurons. Developmental biology 401, 220-235.

136. Lumsden, A., Keynes, R., 1989. Segmental patterns of neuronal development in the chick hindbrain. Nature 337, 424-428.

137. Luquet, S., Perez, F.A., Hnasko, T.S., Palmiter, R.D., 2005. NPY/AgRP neurons are essential for feeding in adult mice but can be ablated in neonates. Science 310, 683685.

138. Maness, P.F., Schachner, M., 2007. Neural recognition molecules of the immunoglobulin superfamily: signaling transducers of axon guidance and neuronal migration. Nat Neurosci 10, 19-26.

139. Mapp, O.M., Walsh, G.S., Moens, C.B., Tada, M., Prince, V.E., 2011. Zebrafish Prickle1b mediates facial branchiomotor neuron migration via a farnesylationdependent nuclear activity. Development 138, 2121-2132.

140. Marin, O., Rubenstein, J.L., 2003. Cell migration in the forebrain. Annual review of neuroscience 26, 441-483.

141. Marin, O., Valiente, M., Ge, X., Tsai, L.H., 2010. Guiding neuronal cell migrations. Cold Spring Harb Perspect Biol 2, a001834. 
142. Marlow, F., Zwartkruis, F., Malicki, J., Neuhauss, S.C., Abbas, L., Weaver, M., Driever, W., Solnica-Krezel, L., 1998. Functional interactions of genes mediating convergent extension, knypek and trilobite, during the partitioning of the eye primordium in zebrafish. Dev Biol 203, 382-399.

143. Mathias, J.R., Zhang, Z., Saxena, M.T., Mumm, J.S., 2014. Enhanced cell-specific ablation in zebrafish using a triple mutant of Escherichia coli nitroreductase. Zebrafish $11,85-97$.

144. May-Simera, H.L., Kai, M., Hernandez, V., Osborn, D.P., Tada, M., Beales, P.L., 2010. Bbs8, together with the planar cell polarity protein Vangl2, is required to establish leftright asymmetry in zebrafish. Developmental biology 345, 215-225.

145. McGuire, S.E., Roman, G., Davis, R.L., 2004. Gene expression systems in Drosophila: a synthesis of time and space. Trends in genetics : TIG 20,384-391.

146. McManus, M.F., Golden, J.A., 2005. Neuronal migration in developmental disorders. J Child Neurol 20, 280-286.

147. Meeker, N.D., Hutchinson, S.A., Ho, L., Trede, N.S., 2007. Method for isolation of PCR-ready genomic DNA from zebrafish tissues. Biotechniques 43, 610, 612, 614.

148. Metcalfe, W.K., Myers, P.Z., Trevarrow, B., Bass, M.B., Kimmel, C.B., 1990. Primary neurons that express the L2/HNK-1 carbohydrate during early development in the zebrafish. Development 110, 491-504.

149. Miyashita, T., Yeo, S.Y., Hirate, Y., Segawa, H., Wada, H., Little, M.H., Yamada, T., Takahashi, N., Okamoto, H., 2004. PlexinA4 is necessary as a downstream target of Islet2 to mediate Slit signaling for promotion of sensory axon branching. Development 131, 3705-3715.

150. Montcouquiol, M., Rachel, R.A., Lanford, P.J., Copeland, N.G., Jenkins, N.A., Kelley, M.W., 2003. Identification of Vangl2 and Scrb1 as planar polarity genes in mammals. Nature 423, 173-177.

151. Moore, J.C., Mulligan, T.S., Torres Yordan, N., Castranova, D., Pham, V.N., Tang, Q., Lobbardi, R., Anselmo, A., Liwski, R.S., Berman, J.N., Sadreyev, R.I., Weinstein, B.M., Langenau, D.M., 2016. T cell immune deficiency in zap70 mutant zebrafish. Mol Cell Biol.

152. Muller, M., Jabs, N., Lorke, D.E., Fritzsch, B., Sander, M., 2003. Nkx6.1 controls migration and axon pathfinding of cranial branchio-motoneurons. Development 130, 5815-5826.

153. Murdoch, J.N., Damrau, C., Paudyal, A., Bogani, D., Wells, S., Greene, N.D., Stanier, P., Copp, A.J., 2014. Genetic interactions between planar cell polarity genes cause diverse neural tube defects in mice. Dis Model Mech 7, 1153-1163. 
154. Nagamune, H., Ohnishi, C., Katsuura, A., Fushitani, K., Whiley, R.A., Tsuji, A., Matsuda, Y., 1996. Intermedilysin, a novel cytotoxin specific for human cells secreted by Streptococcus intermedius UNS46 isolated from a human liver abscess. Infection and immunity 64, 3093-3100.

155. Nakamura, F., Kalb, R.G., Strittmatter, S.M., 2000. Molecular basis of semaphorinmediated axon guidance. Journal of neurobiology 44, 219-229.

156. Nambiar, R.M., Ignatius, M.S., Henion, P.D., 2007. Zebrafish colgate/hdac1 functions in the non-canonical Wnt pathway during axial extension and in Wnt-independent branchiomotor neuron migration. Mechanisms of development 124, 682-698.

157. Neuhauss, S.C., Biehlmaier, O., Seeliger, M.W., Das, T., Kohler, K., Harris, W.A., Baier, H., 1999. Genetic disorders of vision revealed by a behavioral screen of 400 essential loci in zebrafish. The Journal of neuroscience : the official journal of the Society for Neuroscience 19, 8603-8615.

158. Niculescu, C., Ganguli-Indra, G., Pfister, V., Dupe, V., Messaddeq, N., De Arcangelis, A., Georges-Labouesse, E., 2011. Conditional ablation of integrin alpha- 6 in mouse epidermis leads to skin fragility and inflammation. European journal of cell biology 90 , 270-277.

159. Noden, D.M., 1983. The role of the neural crest in patterning of avian cranial skeletal, connective, and muscle tissues. Developmental biology 96, 144-165.

160. Nusslein-Volhard, C., Wieschaus, E., Kluding, H., 1984. Mutations affecting the pattern of the larval cuticle inDrosophila melanogaster : I. Zygotic loci on the second chromosome. Wilehm Roux Arch Dev Biol 193, 267-282.

161. Odenthal, J., Haffter, P., Vogelsang, E., Brand, M., van Eeden, F.J., Furutani-Seiki, M., Granato, M., Hammerschmidt, M., Heisenberg, C.P., Jiang, Y.J., Kane, D.A., Kelsh, R.N., Mullins, M.C., Warga, R.M., Allende, M.L., Weinberg, E.S., Nusslein-Volhard, C., 1996. Mutations affecting the formation of the notochord in the zebrafish, Danio rerio. Development 123, 103-115.

162. Ohkura, K., Kawaguchi, Y., Tabata, A., Yamamoto, A., Shinohara, Y., Nagamune, H., Hori, H., 2012. Molecular profiles of cholesterol-dependent cytolysin family-derived 11 mer regions. Anticancer research 32, 2343-2346.

163. Orger, M.B., Smear, M.C., Anstis, S.M., Baier, H., 2000. Perception of Fourier and non-Fourier motion by larval zebrafish. Nature neuroscience 3, 1128-1133.

164. Pan, X., Sittaramane, V., Gurung, S., Chandrasekhar, A., 2014. Structural and temporal requirements of Wnt/PCP protein Vangl2 function for convergence and extension movements and facial branchiomotor neuron migration in zebrafish. Mechanisms of development 131, 1-14. 
165. Park, M., Moon, R.T., 2002. The planar cell-polarity gene stbm regulates cell behaviour and cell fate in vertebrate embryos. Nature cell biology 4, 20-25.

166. Parnavelas, J.G., Alifragis, P., Nadarajah, B., 2002. The origin and migration of cortical neurons. Progress in brain research 136, 73-80.

167. Parsons, M.J., Pollard, S.M., Saude, L., Feldman, B., Coutinho, P., Hirst, E.M., Stemple, D.L., 2002. Zebrafish mutants identify an essential role for laminins in notochord formation. Development 129, 3137-3146.

168. Paulus, J.D., Halloran, M.C., 2006. Zebrafish bashful/laminin-alpha 1 mutants exhibit multiple axon guidance defects. Developmental dynamics : an official publication of the American Association of Anatomists 235, 213-224.

169. Pavlou, O., Theodorakis, K., Falk, J., Kutsche, M., Schachner, M., Faivre-Sarrailh, C., Karagogeos, D., 2002. Analysis of interactions of the adhesion molecule TAG-1 and its domains with other immunoglobulin superfamily members. Molecular and cellular neurosciences 20, 367-381.

170. Pearlman, A.L., Faust, P.L., Hatten, M.E., Brunstrom, J.E., 1998. New directions for neuronal migration. Current opinion in neurobiology $8,45-54$.

171. Phillips, J.B., Blanco-Sanchez, B., Lentz, J.J., Tallafuss, A., Khanobdee, K., Sampath, S., Jacobs, Z.G., Han, P.F., Mishra, M., Titus, T.A., Williams, D.S., Keats, B.J., Washbourne, P., Westerfield, M., 2011. Harmonin (Ush1c) is required in zebrafish Muller glial cells for photoreceptor synaptic development and function. Dis Model Mech 4, 786-800.

172. Pisharath, H., Parsons, M.J., 2009. Nitroreductase-mediated cell ablation in transgenic zebrafish embryos. Methods in molecular biology (Clifton, N.J.) 546, 133-143.

173. Pocock, R., Mione, M., Hussain, S., Maxwell, S., Pontecorvi, M., Aslam, S., Gerrelli, D., Sowden, J.C., Woollard, A., 2008. Neuronal function of Tbx20 conserved from nematodes to vertebrates. Developmental biology 317, 671-685.

174. Pogoda, H.M., Sternheim, N., Lyons, D.A., Diamond, B., Hawkins, T.A., Woods, I.G., Bhatt, D.H., Franzini-Armstrong, C., Dominguez, C., Arana, N., Jacobs, J., Nix, R., Fetcho, J.R., Talbot, W.S., 2006. A genetic screen identifies genes essential for development of myelinated axons in zebrafish. Developmental biology 298, 118-131.

175. Portugues, R., Engert, F., 2009. The neural basis of visual behaviors in the larval zebrafish. Current opinion in neurobiology 19, 644-647.

176. Powell, S.K., Kleinman, H.K., 1997. Neuronal laminins and their cellular receptors. The international journal of biochemistry \& cell biology 29, 401-414.

177. Qu, Y., Glasco, D.M., Zhou, L., Sawant, A., Ravni, A., Fritzsch, B., Damrau, C., Murdoch, J.N., Evans, S., Pfaff, S.L., Formstone, C., Goffinet, A.M., Chandrasekhar, 
A., Tissir, F., 2010. Atypical cadherins Celsr1-3 differentially regulate migration of facial branchiomotor neurons in mice. The Journal of neuroscience : the official journal of the Society for Neuroscience 30, 9392-9401.

178. Rakic, P., 1974. Neurons in rhesus monkey visual cortex: systematic relation between time of origin and eventual disposition. Science 183, 425-427.

179. Raper, J.A., 2000. Semaphorins and their receptors in vertebrates and invertebrates. Current opinion in neurobiology $10,88-94$.

180. Rebman, J.K., Kirchoff, K.E., Walsh, G.S., 2016. Cadherin-2 Is Required Cell Autonomously for Collective Migration of Facial Branchiomotor Neurons. PLoS One 11, e0164433.

181. Reyes, R., Haendel, M., Grant, D., Melancon, E., Eisen, J.S., 2004. Slow degeneration of zebrafish Rohon-Beard neurons during programmed cell death. Developmental dynamics : an official publication of the American Association of Anatomists 229, 3041.

182. Ribera, A.B., Nusslein-Volhard, C., 1998. Zebrafish touch-insensitive mutants reveal an essential role for the developmental regulation of sodium current. The Journal of neuroscience : the official journal of the Society for Neuroscience 18, 9181-9191.

183. Rivas, R.J., Hatten, M.E., 1995. Motility and cytoskeletal organization of migrating cerebellar granule neurons. The Journal of neuroscience : the official journal of the Society for Neuroscience 15, 981-989.

184. Rohrschneider, M.R., Elsen, G.E., Prince, V.E., 2007. Zebrafish Hoxb1a regulates multiple downstream genes including prickle1b. Developmental biology 309, 358-372.

185. Ross, L.S., Parrett, T., Easter, S.S., Jr., 1992. Axonogenesis and morphogenesis in the embryonic zebrafish brain. The Journal of neuroscience : the official journal of the Society for Neuroscience 12, 467-482.

186. Rossi, A., Kontarakis, Z., Gerri, C., Nolte, H., Holper, S., Kruger, M., Stainier, D.Y., 2015. Genetic compensation induced by deleterious mutations but not gene knockdowns. Nature 524, 230-233.

187. Roszko, I., Sawada, A., Solnica-Krezel, L., 2009. Regulation of convergence and extension movements during vertebrate gastrulation by the Wnt/PCP pathway. Seminars in cell \& developmental biology $20,986-997$.

188. Saito, M., Iwawaki, T., Taya, C., Yonekawa, H., Noda, M., Inui, Y., Mekada, E., Kimata, Y., Tsuru, A., Kohno, K., 2001. Diphtheria toxin receptor-mediated conditional and targeted cell ablation in transgenic mice. Nature biotechnology 19, 746-750.

189. Sakai, J.A., Halloran, M.C., 2006. Semaphorin 3d guides laterality of retinal ganglion cell projections in zebrafish. Development 133, 1035-1044. 
190. Sankrithi, N.S., O'Malley, D.M., 2010. Activation of a multisensory, multifunctional nucleus in the zebrafish midbrain during diverse locomotor behaviors. Neuroscience 166, 970-993.

191. Sassen, W.A., Lehne, F., Russo, G., Wargenau, S., Dubel, S., Koster, R.W., 2017. Embryonic zebrafish primary cell culture for transfection and live cellular and subcellular imaging. Developmental biology 430, 18-31.

192. Savvaki, M., Panagiotaropoulos, T., Stamatakis, A., Sargiannidou, I., Karatzioula, P., Watanabe, K., Stylianopoulou, F., Karagogeos, D., Kleopa, K.A., 2008. Impairment of learning and memory in TAG-1 deficient mice associated with shorter CNS internodes and disrupted juxtaparanodes. Molecular and cellular neurosciences 39, 478-490.

193. Schlatter, M.C., Buhusi, M., Wright, A.G., Maness, P.F., 2008. CHL1 promotes Sema3A-induced growth cone collapse and neurite elaboration through a motif required for recruitment of ERM proteins to the plasma membrane. Journal of neurochemistry 104, 731-744.

194. Serafini, T., Colamarino, S.A., Leonardo, E.D., Wang, H., Beddington, R., Skarnes, W.C., Tessier-Lavigne, M., 1996. Netrin-1 is required for commissural axon guidance in the developing vertebrate nervous system. Cell 87, 1001-1014.

195. Serafini, T., Kennedy, T.E., Galko, M.J., Mirzayan, C., Jessell, T.M., Tessier-Lavigne, M., 1994. The netrins define a family of axon outgrowth-promoting proteins homologous to C. elegans UNC-6. Cell 78, 409-424.

196. Severi, K.E., Portugues, R., Marques, J.C., O'Malley, D.M., Orger, M.B., Engert, F., 2014. Neural control and modulation of swimming speed in the larval zebrafish. Neuron 83, 692-707.

197. Sharma, A., Gokulchandran, N., Chopra, G., Kulkarni, P., Lohia, M., Badhe, P., Jacob, V.C., 2012. Administration of autologous bone marrow-derived mononuclear cells in children with incurable neurological disorders and injury is safe and improves their quality of life. Cell transplantation 21 Suppl 1, S79-90.

198. Sittaramane, V., Pan, X., Glasco, D.M., Huang, P., Gurung, S., Bock, A., Li, S., Wang, H., Kawakami, K., Matise, M.P., Chandrasekhar, A., 2013. The PCP protein Vangl2 regulates migration of hindbrain motor neurons by acting in floor plate cells, and independently of cilia function. Developmental biology 382, 400-412.

199. Sittaramane, V., Sawant, A., Wolman, M.A., Maves, L., Halloran, M.C., Chandrasekhar, A., 2009. The cell adhesion molecule Tag1, transmembrane protein Stbm/Vangl2, and Lamininalpha1 exhibit genetic interactions during migration of facial branchiomotor neurons in zebrafish. Developmental biology 325, 363-373.

200. Smith, A., Robinson, V., Patel, K., Wilkinson, D.G., 1997. The EphA4 and EphB1 receptor tyrosine kinases and ephrin-B2 ligand regulate targeted migration of branchial neural crest cells. Current biology : CB 7, 561-570. 
201. Solecki, D.J., Govek, E.E., Tomoda, T., Hatten, M.E., 2006. Neuronal polarity in CNS development. Genes Dev 20, 2639-2647.

202. Solnica-Krezel, L., Stemple, D.L., Mountcastle-Shah, E., Rangini, Z., Neuhauss, S.C., Malicki, J., Schier, A.F., Stainier, D.Y., Zwartkruis, F., Abdelilah, S., Driever, W., 1996. Mutations affecting cell fates and cellular rearrangements during gastrulation in zebrafish. Development 123, 67-80.

203. Soltani, C.E., Hotze, E.M., Johnson, A.E., Tweten, R.K., 2007. Specific proteinmembrane contacts are required for prepore and pore assembly by a cholesteroldependent cytolysin. The Journal of biological chemistry 282, 15709-15716.

204. Song, H., Hu, J., Chen, W., Elliott, G., Andre, P., Gao, B., Yang, Y., 2010. Planar cell polarity breaks bilateral symmetry by controlling ciliary positioning. Nature 466, 378382.

205. Song, M.R., 2007. Moving cell bodies: understanding the migratory mechanism of facial motor neurons. Archives of pharmacal research 30, 1273-1282.

206. Stainier, D.Y.R., Raz, E., Lawson, N.D., Ekker, S.C., Burdine, R.D., Eisen, J.S., Ingham, P.W., Schulte-Merker, S., Yelon, D., Weinstein, B.M., Mullins, M.C., Wilson, S.W., Ramakrishnan, L., Amacher, S.L., Neuhauss, S.C.F., Meng, A., Mochizuki, N., Panula, P., Moens, C.B., 2017. Guidelines for morpholino use in zebrafish. PLoS Genet 13, e1007000.

207. Stockinger, P., Maitre, J.L., Heisenberg, C.P., 2011. Defective neuroepithelial cell cohesion affects tangential branchiomotor neuron migration in the zebrafish neural tube. Development 138, 4673-4683.

208. Stoeckli, E.T., 2004. Ig superfamily cell adhesion molecules in the brain. Handb Exp Pharmacol, 373-401.

209. Stoeckli, E.T., Landmesser, L.T., 1995. Axonin-1, Nr-CAM, and Ng-CAM play different roles in the in vivo guidance of chick commissural neurons. Neuron 14, 1165-1179.

210. Sun, C., Wu, J., Liu, S., Li, H., Zhang, S., 2013. Zebrafish CD59 has both bacterialbinding and inhibiting activities. Developmental and comparative immunology 41, 178188.

211. Suter, D.M., Pollerberg, G.E., Buchstaller, A., Giger, R.J., Dreyer, W.J., Sonderegger, P., 1995. Binding between the neural cell adhesion molecules axonin-1 and $\mathrm{Nr}-$ CAM/Bravo is involved in neuron-glia interaction. The Journal of cell biology 131 , 1067-1081.

212. Suzuki, S.C., Takeichi, M., 2008. Cadherins in neuronal morphogenesis and function. Development, growth \& differentiation 50 Suppl 1, S119-130. 
213. Tabata, A., Ohkura, K., Ohkubo, Y., Tomoyasu, T., Ohkuni, H., Whiley, R.A., Nagamune, H., 2014. The diversity of receptor recognition in cholesterol-dependent cytolysins. Microbiology and immunology 58, 155-171.

214. Taniguchi, M., Yuasa, S., Fujisawa, H., Naruse, I., Saga, S., Mishina, M., Yagi, T., 1997. Disruption of semaphorin III/D gene causes severe abnormality in peripheral nerve projection. Neuron 19, 519-530.

215. Tazir, M., Nouioua, S., Magy, L., Huehne, K., Assami, S., Urtizberea, A., Grid, D., Hamadouche, T., Rautenstrauss, B., Vallat, J.M., 2009. Phenotypic variability in giant axonal neuropathy. Neuromuscul Disord 19, 270-274.

216. Tessier-Lavigne, M., Goodman, C.S., 1996. The molecular biology of axon guidance. Science 274, 1123-1133.

217. Thompson, H., Andrews, W., Parnavelas, J.G., Erskine, L., 2009. Robo2 is required for Slit-mediated intraretinal axon guidance. Developmental biology 335, 418-426.

218. Tongiorgi, E., Bernhardt, R.R., Schachner, M., 1995. Zebrafish neurons express two L1-related molecules during early axonogenesis. Journal of neuroscience research 42 , 547-561.

219. Tzimourakas, A., Giasemi, S., Mouratidou, M., Karagogeos, D., 2007. Structurefunction analysis of protein complexes involved in the molecular architecture of juxtaparanodal regions of myelinated fibers. Biotechnol J 2, 577-583.

220. Uemura, O., Okada, Y., Ando, H., Guedj, M., Higashijima, S., Shimazaki, T., Chino, N., Okano, H., Okamoto, H., 2005. Comparative functional genomics revealed conservation and diversification of three enhancers of the isl1 gene for motor and sensory neuron-specific expression. Developmental biology 278, 587-606.

221. Vajsar, J., Schachter, H., 2006. Walker-Warburg syndrome. Orphanet journal of rare diseases 1, 29.

222. Vanderlaan, G., Tyurina, O.V., Karlstrom, R.O., Chandrasekhar, A., 2005. Gli function is essential for motor neuron induction in zebrafish. Developmental biology 282, $550-$ 570 .

223. Veeman, M.T., Axelrod, J.D., Moon, R.T., 2003. A second canon. Functions and mechanisms of beta-catenin-independent Wnt signaling. Developmental cell 5, 367377.

224. Vivancos, V., Chen, P., Spassky, N., Qian, D., Dabdoub, A., Kelley, M., Studer, M., Guthrie, S., 2009. Wnt activity guides facial branchiomotor neuron migration, and involves the PCP pathway and JNK and ROCK kinases. Neural development 4, 7.

225. Wada, H., Iwasaki, M., Sato, T., Masai, I., Nishiwaki, Y., Tanaka, H., Sato, A., Nojima, Y., Okamoto, H., 2005. Dual roles of zygotic and maternal Scribble1 in neural 
migration and convergent extension movements in zebrafish embryos. Development 132, 2273-2285.

226. Wada, H., Tanaka, H., Nakayama, S., Iwasaki, M., Okamoto, H., 2006. Frizzled3a and Celsr2 function in the neuroepithelium to regulate migration of facial motor neurons in the developing zebrafish hindbrain. Development 133, 4749-4759.

227. Walsh, G.S., Grant, P.K., Morgan, J.A., Moens, C.B., 2011. Planar polarity pathway and Nance-Horan syndrome-like $1 \mathrm{~b}$ have essential cell-autonomous functions in neuronal migration. Development 138, 3033-3042.

228. Wan, H., Korzh, S., Li, Z., Mudumana, S.P., Korzh, V., Jiang, Y.J., Lin, S., Gong, Z., 2006. Analyses of pancreas development by generation of gfp transgenic zebrafish using an exocrine pancreas-specific elastaseA gene promoter. Experimental cell research 312, 1526-1539.

229. Wanner, S.J., Prince, V.E., 2013. Axon tracts guide zebrafish facial branchiomotor neuron migration through the hindbrain. Development 140, 906-915.

230. Warren, J.T., Jr., Chandrasekhar, A., Kanki, J.P., Rangarajan, R., Furley, A.J., Kuwada, J.Y., 1999. Molecular cloning and developmental expression of a zebrafish axonal glycoprotein similar to TAG-1. Mechanisms of development 80, 197-201.

231. Westerfield, M., 1995. The Zebrafish Book.

232. Whitlock, K.E., Westerfield, M., 2000. The olfactory placodes of the zebrafish form by convergence of cellular fields at the edge of the neural plate. Development 127, 36453653.

233. Wickham, S.E., Hotze, E.M., Farrand, A.J., Polekhina, G., Nero, T.L., Tomlinson, S., Parker, M.W., Tweten, R.K., 2011. Mapping the intermedilysin-human CD59 receptor interface reveals a deep correspondence with the binding site on CD59 for complement binding proteins C8alpha and C9. The Journal of biological chemistry 286, 20952-20962.

234. Williams, J.A., Barrios, A., Gatchalian, C., Rubin, L., Wilson, S.W., Holder, N., 2000. Programmed cell death in zebrafish rohon beard neurons is influenced by TrkC1/NT-3 signaling. Developmental biology 226, 220-230.

235. Williams, S.E., Mann, F., Erskine, L., Sakurai, T., Wei, S., Rossi, D.J., Gale, N.W., Holt, C.E., Mason, C.A., Henkemeyer, M., 2003. Ephrin-B2 and EphB1 mediate retinal axon divergence at the optic chiasm. Neuron 39, 919-935.

236. Wolman, M.A., Liu, Y., Tawarayama, H., Shoji, W., Halloran, M.C., 2004. Repulsion and attraction of axons by semaphorin3D are mediated by different neuropilins in vivo. The Journal of neuroscience : the official journal of the Society for Neuroscience 24, 8428-8435. 
237. Wolman, M.A., Regnery, A.M., Becker, T., Becker, C.G., Halloran, M.C., 2007. Semaphorin3D regulates axon axon interactions by modulating levels of $L 1$ cell adhesion molecule. The Journal of neuroscience : the official journal of the Society for Neuroscience 27, 9653-9663.

238. Wolman, M.A., Sittaramane, V.K., Essner, J.J., Yost, H.J., Chandrasekhar, A., Halloran, M.C., 2008. Transient axonal glycoprotein-1 (TAG-1) and laminin-alpha1 regulate dynamic growth cone behaviors and initial axon direction in vivo. Neural development 3, 6 .

239. Yamamoto, M., Boyer, A.M., Crandall, J.E., Edwards, M., Tanaka, H., 1986. Distribution of stage-specific neurite-associated proteins in the developing murine nervous system recognized by a monoclonal antibody. The Journal of neuroscience : the official journal of the Society for Neuroscience 6, 3576-3594.

240. Yang, C.T., Sengelmann, R.D., Johnson, S.L., 2004. Larval melanocyte regeneration following laser ablation in zebrafish. The Journal of investigative dermatology 123, 924-929.

241. Yokoyama, N., Romero, M.I., Cowan, C.A., Galvan, P., Helmbacher, F., Charnay, P., Parada, L.F., Henkemeyer, M., 2001. Forward signaling mediated by ephrin-B3 prevents contralateral corticospinal axons from recrossing the spinal cord midline. Neuron 29, 85-97.

242. Zhao, X.F., Ellingsen, S., Fjose, A., 2009. Labelling and targeted ablation of specific bipolar cell types in the zebrafish retina. BMC Neurosci 10, 107. 


\section{Vita}

Suman Gurung was born May 20th, 1987 in Nepal to Krishna Raj and Goma Gurung. Suman came to the US in 2007 after completing his high school in his home country. He received his combined Bachelor's/Master's degree in Biotechnology from the College of Agriculture, Biotechnology, and Natural Resources at the University of Nevada, Reno and was awarded the Outstanding Graduate Student in Biotechnology. This work was completed under the guidance of Dr. Anand Chandrasekhar at the University of Missouri, Columbia. Suman will join the lab of Dr. Saulius Sumanas at the Cincinnati Children's Hospital Medical Center in June 2018. 